Aus dem Institut für Allgemeinmedizin

(Prof. Dr. med. E. Hummers-Pradier)

der Medizinischen Fakultät der Universität Göttingen

\title{
Schlaf um jeden Preis
}

Eine Befragung älterer Patienten

zu den Erfahrungen mit Schlaf- und Beruhigungsmitteln

im Krankenhaus und zu Hause

\author{
INAUGURAL - DISSERTATION \\ zur Erlangung des Doktorgrades \\ der Medizinischen Fakultät der
}

Georg-August-Universität zu Göttingen

vorgelegt von

\section{Freya Sophie Neukirchen}

aus

Bremen

Göttingen 2018 
Dekan: $\quad$ Prof. Dr. rer. nat. H. K. Kroemer

Referent: Prof. Dr. disc. pol. W. Himmel

Ko-Referent/in: $\quad$ PD Dr. Bernhard Kis

Drittreferent/in

Datum der mündlichen Prüfung: $\quad$ 24.10.2019 
Hiermit erkläre ich, die Dissertation mit dem Titel „Schlaf um jeden Preis“ eigenständig angefertigt und keine andere als die von mir angegebenen Quellen und Hilfsmittel verwendet zu haben.

Göttingen, den

Unterschrift: 


\section{Inhaltsverzeichnis}

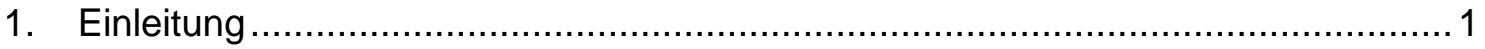

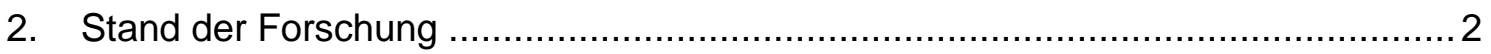

2.1 Schlafstörungen und nicht erholsamer Schlaf ............................................ 2

2.1.1 Definition und Diagnostik laut deutscher S3-Leitlinie ....................... 2

2.1.2 Vergleich mit anderen Leitlinien und Empfehlungen ......................... 2

2.1.3 Vorkommen und Häufigkeit von Schlafstörungen ............................ 3

2.2 Pharmakologische Therapie bei Schlafstörungen mit Benzodiazepinen und

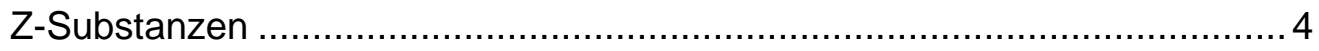

2.2.1 Exkurs: Historie der Benzodiazepine und Z-Substanzen .................. 4

2.2.2 Leitliniengerechte Therapie ....................................................... 5

2.2.3 Empfehlungen für ältere Patienten laut PRISCUS-Liste.....................6 6

2.2.4 Wirkungen und Anwendungsgebiete .......................................... 7

2.2.5 Pharmakologie von Benzodiazepinen und Z-Substanzen bei älteren Patienten ...................................................................... 7

2.2.6 Nebenwirkungen durch die Einnahme von Benzodiazepinen und ZSubstanzen

2.2.7 Toleranz- und Abhängigkeitspotenzial von Benzodiazepinen und ZSubstanzen .................................................................... 11

2.2.8 Entzugserscheinungen nach der Einnahme von Benzodiazepinen und Z-Substanzen ................................................................... 12

2.2.9 Häufigkeit der Einnahme von Benzodiazepinen und Z-Substanzen. 13

2.3 Die Patientenperspektive auf den Gebrauch von Benzodiazepinen und Z-

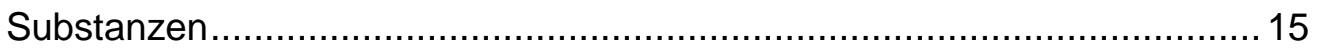

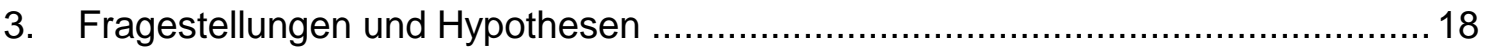

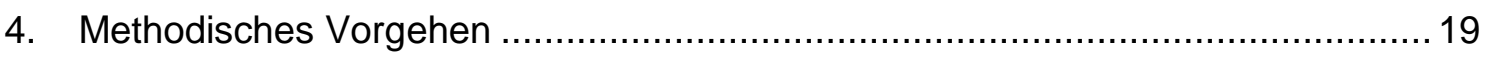

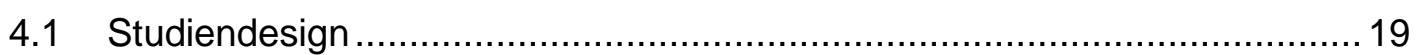

4.2 Stellenwert der Untersuchung im Gesamtprojekt .................................. 19

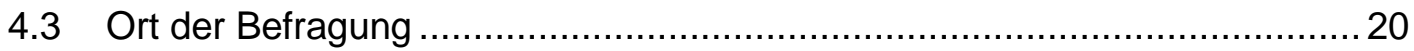

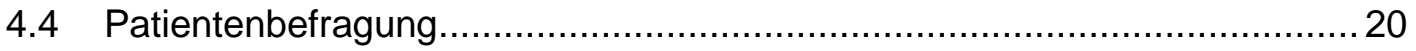

4.4.1 Standardisierte Befragung ............................................... 21

4.4.2 Entwicklung des Fragebogens ............................................ 21

4.4.3 Pilotstudie ........................................................................... 22

4.4.4 Der Fragebogen ..................................................................... 22

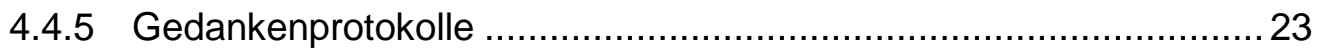

4.4.6 Ein- und Ausschlusskriterien .................................................... 23

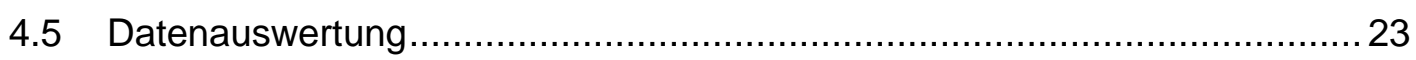

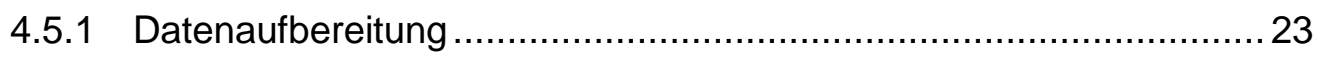




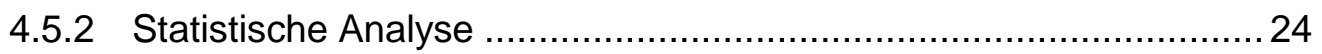

4.6 Datenschutz und Genehmigung durch die Ethik-Kommission ................... 25

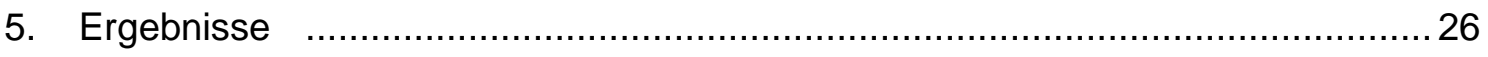

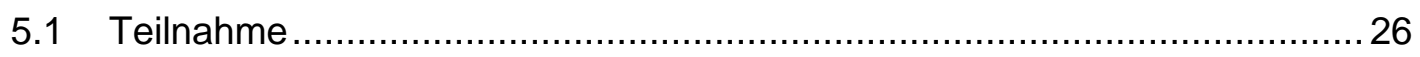

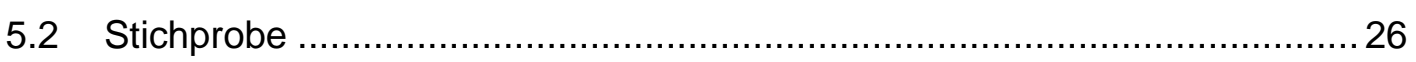

5.3 Einnahme von Schlaf- oder Beruhigungsmitteln während des Krankenhausaufenthalts

5.4 Wahrgenommene Verbesserungen und Nebenwirkungen durch die Einnahme der Schlaf- und Beruhigungsmittel während des stationären Aufenthalts

5.5 Einnahme von Schlaf- oder Beruhigungsmitteln vor dem Krankenhausaufenthalt

5.6 Erfahrungsberichte der Patienten laut Gedankenprotokoll

5.7 Dokumentation der Schlaf- oder Beruhigungsmittel in der Patientenakte.

5.8 Vergleich der Patientenakte mit der Patientenaussage

5.9 Einflussfaktoren auf den Wunsch der Weitereinnahme des stationär erhaltenen Schlaf- oder Beruhigungsmittels.

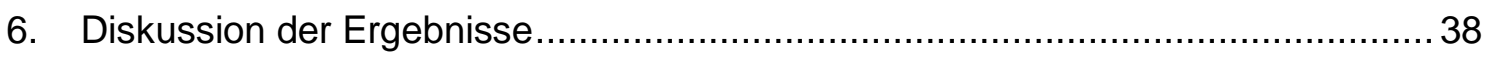

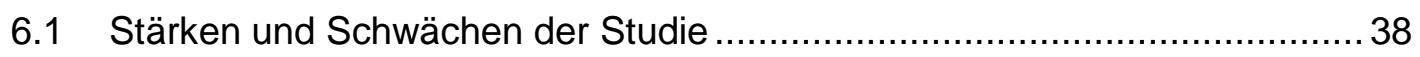

6.1.1 Persönliche Befragung ........................................................ 38

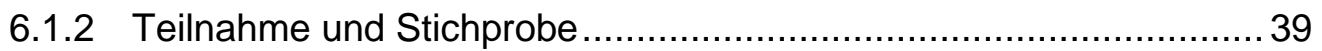

6.1.3 Demenz und Orientierungslosigkeit als Ausschlusskriterien ........... 40

6.1.4 Berücksichtigung spezifischer Stoffklassen ................................ 41

6.1.5 Indifferente Antworten der Patienten.......................................... 41

6.2 Einnahmeprävalenz während des stationären Aufenthalts ......................... 42

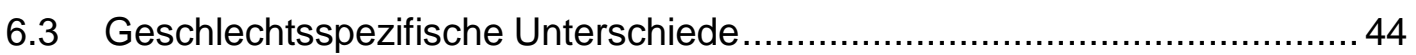

6.4 Relevanz der Vorerfahrungen mit Schlaf- und Beruhigungsmitteln ............ 45

6.5 Wunsch der weiteren Einnahme nach Entlassung .................................. 46

6.6 Freiverkäufliche und ärztlich verordnete Medikamente ............................. 46

6.7 Differenzen zwischen den Fachabteilungen ......................................... 47

6.8 Verlässlichkeit der Patientenaussagen im Vergleich zu den Angaben in den Patientenakten

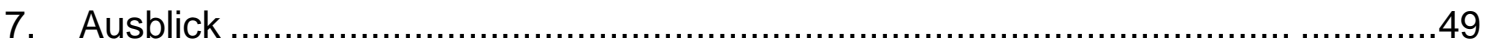

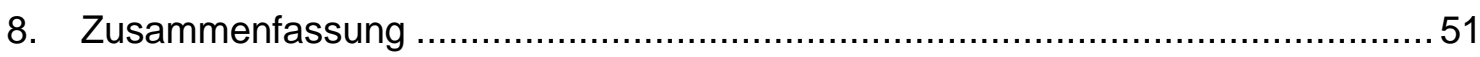

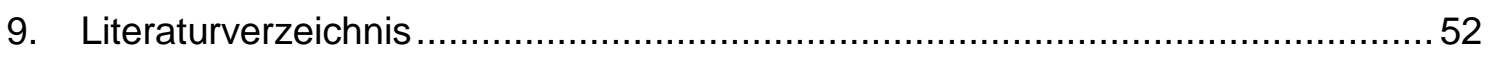

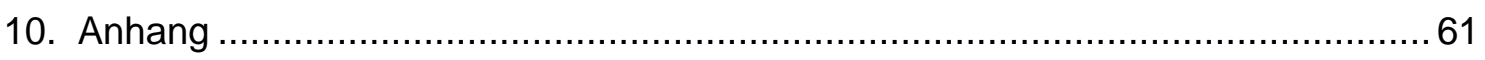




\section{Einleitung}

„Early to bed and early to rise, makes a man healthy, wealthy, and wise” (,Früh zu Bett und früh aufstehen, macht einen Menschen gesund, wohlhabend und weise"). Dieser Ausspruch wird Benjamin Franklin, einer der Gründerväter der Vereinigten Staaten von Amerika, zugeschrieben. Viele ältere Menschen empfinden allerdings ein zu frühes Aufwachen am Morgen - anders als der amerikanische Unabhängigkeitskämpfer - als störend und haben gleichzeitig Schwierigkeiten, am Abend in den Schlaf zu kommen. Statt gesund, wohlhabend bzw. tatkräftig und weise, fühlen sie sich schlapp, abgeschlagen und leistungsgemindert. Chronische Erkrankungen, Schmerzen im Bewegungsapparat, fehlende geistige und körperliche Beanspruchung am Tage oder auch eine belastete Gemütslage sind Gründe für einen nicht erholsamen Schlaf. Dies betrifft zumeist die häusliche Situation, kann aber auch in der akuten Situation einer stationären Behandlung im Krankenhaus zum Problem werden. Speziell der veränderte Tagesablauf auf einer Station, der von den medizinischen Abläufen des Krankenhauses bestimmt wird, bringt den gewohnten Rhythmus durcheinander. Die vorgegebenen Weck-, Essens- und Schlafzeiten, gegebenenfalls Bettlägerigkeit, Schmerzen, Sorgen, Angst und die fehlende körperliche Aktivität im Krankenhaus können Probleme des Einund Durchschlafens auslösen bzw. verstärken und auch tagsüber zu einer inneren Unruhe führen.

Belastet von dieser Situation sowohl im Krankenhaus als auch zu Hause treten nicht wenige Patienten* an ihre Ärzte heran, um ihre Beschwerden mit der Einnahme von Schlaf- und Beruhigungsmittel zu lindern. Zu den am häufigsten verordneten Medikamenten gehören die verschreibungspflichtigen Benzodiazepine und Z-Substanzen aus der Gruppe der Hypnosedativa. Diese Wirkstoffe gelten als sehr effektiv, da sie schnell schlafinduzierend und schlafverlängernd wirken. Sie haben aber den Nachteil, dass sie insbesondere bei Patienten höheren Alters gegebenenfalls zu Gewöhnungseffekten, Abhängigkeit und starken Nebenwirkungen führen. So können beispielsweise medikamentös bedingte kognitive Einbußen oder muskelrelaxierende Wirkungen Stürze mit Knochenbrüchen verursachen. In der stationären Situation sollen Patienten mit Hilfe von Benzodiazepinen und Z-Substanzen kurzfristig von Schlafproblemen befreit werden. Langfristig kann die Einnahme aber durch ambulante Weiterverordnungen nach der Entlassung aus dem Krankenhaus zu einem Problem werden.

In dieser Studie soll diese Problematik aus der Perspektive älterer Patienten untersucht werden. Dafür sollen deren Erfahrungen mit Schlaf- und Beruhigungsmitteln, die Motive für die Einnahme und der persönlich empfundene Nutzen bzw. Schaden dieser Medikamente erhoben werden.

* Im Folgenden wird - um den Lesefluss nicht zu unterbrechen - immer die männliche Form verwendet, die stets die weibliche einschließt. 


\section{Stand der Forschung}

\subsection{Schlafstörungen und nicht erholsamer Schlaf}

Im Folgenden werden die Begriffe Schlafstörungen und Insomnien erklärt, Leitsymptome und Klassifikationen vorgestellt und Behandlungsoptionen erläutert.

\subsubsection{Definition und Diagnostik laut deutscher S3-Leitlinie}

Laut der S3-Leitlinie „Nicht erholsamer Schlaf/Schlafstörungen“ der Deutschen Gesellschaft für Schlafforschung und Schlafmedizin (DGSM) inklusive der aktuellen Ergänzung der Leitlinie von 2016 sind Insomnien Störungen des Ein- und Durchschlafens; wesentliche Charakteristika von nicht erholsamem Schlaf können Hypersomnie (Schlafsucht) und Tagesschläfrigkeit sein (DGSM 2009; DGSM 2017).

Im DSM-IV-System (American Psychiatric Association = APA; 1994) werden einer primären Insomnie die Leitsymptome Ein- und Durchschlafstörungen und nicht erholsamer Schlaf zugeordnet. Dagegen definiert das ICSD-R-System (American Academy of Sleep Medicine; 2001) Insomnie als zu wenig bzw. nicht erholsamen Schlaf. Auf diese Definition bezieht sich insbesondere die bereits erwähnte S3-Leitlinie.

Zur Diagnostik von Insomnie empfiehlt die DGSM vor allem eine schlafmedizinische Anamnese inklusive Schlaffragebögen und Schlaftagebüchern. Auch nach der Einnahme von schlafstörenden Substanzen soll gefragt werden. Auswahl, Art und Umfang der Anamnese mögen bedarfsgerecht und wirtschaftlich durch den Arzt entschieden werden. Somit sind die Diagnoseverfahren wenig standardisiert; dies wird laut S3-Leitlinie damit begründet, dass Schlafstörungen vielgestaltig und sehr individuell aufträten.

Schlafprobleme kommen primär/nicht-organisch oder sekundär begleitend zu einer Vielzahl von anderen Erkrankungen vor, auf die an dieser Stelle nicht im Detail eingegangen werden soll.

\subsubsection{Vergleich mit anderen Leitlinien und Empfehlungen}

Vergleicht man die S3-Leitlinie der DGSM mit anderen internationalen Leitlinien, zeigt sich ein recht einheitliches Bild:

Die Leitlinie der European Medicines Agency von 2011 zur Behandlung von Insomnien definiert Insomnien entgegen der deutschen S3-Leitlinie nach den Kriterien des oben erwähnten DSM-IV-Systems (APA). Eine primäre Insomnie sei demnach charakterisiert durch eines oder mehrere der folgenden Hauptsymptome über mindestens einen Monat (European Medicines Agency 2011):

- Schwierigkeiten bei der Schlafinitiation 
- Störungen beim Durchschlafen (häufiges Aufwachen oder lange Wachphasen)

- vorzeitiges Erwachen

- Gefühl eines nicht erholsamen Schlafs

- nachfolgende Einschränkung der Leistungsfähigkeit am Tag.

Die Empfehlungen zur Diagnostik von Schlafstörungen entsprechen jedoch weitgehend der deutschen S3-Leitlinie. Zu berücksichtigen sei aber, dass Tests im Schlaflabor zwar objektivierte, quantitative Ergebnisse zu Schlafunterbrechungen und -verzögerung lieferten, durch die ungewohnte künstliche Schlafumgebung jedoch durch den Patienten subjektiv verfälscht würden und nicht in jedem Fall als Methode der Wahl angemessen seien (European Medicines Agency 2011). Die klinische Leitlinie zum Umgang mit chronischer Insomnie des „Journal of Clinical Sleep Medicine" empfiehlt wie die deutsche S3-Leitlinie ebenfalls Fragebögen und Schlaftagebücher zur Diagnostik; gleichermaßen formuliert dies die brasilianische Leitlinie der Brazilian Sleep Association. Zusätzlich werden psychologische Screeningtests und Befragungen der Bettpartner genannt (Schutte-Rodin et al. 2008; Pinto Jr. et al. 2010).

\subsubsection{Vorkommen und Häufigkeit von Schlafstörungen}

Die Angaben zur Prävalenz von Schlafstörungen in der Bevölkerung schwanken in der Fachliteratur signifikant:

Die erwähnte S3-Leitlinie der DGSM bezieht sich auf ein klinisches Review von 2002 aus den USA. Ohayon analysierte dort über 50 Studien nach verschiedenen Kriterien. Fünf dieser Studien verwendeten die oben aufgeführten Kriterien der DSM-IV-Klassifikation. Unter den über 20400 Studienteilnehmern aus Kanada, Frankreich, Großbritannien, Deutschland und Italien im Alter von $\geq 15$ Jahren aus der Allgemeinbevölkerung ergab sich eine Prävalenz von ca. $6 \%$. Genau diese Prävalenz, nämlich $6 \%$, galt auch für die deutsche Teilstudie mit 4115 Teilnehmern. Frauen waren häufiger von nicht erholsamem Schlaf betroffen als Männer $(8,2 \%$ versus 5,6\%). In den meisten der analysierten Studien nahm die Häufigkeit von Schlafproblemen im höheren Alter zu (Ohayon 2002).

Basierend auf Patientenauskünften wird im Gesundheitssurvey des Robert Koch-Instituts (2005) festgestellt, dass $27 \%$ der Frauen und $14 \%$ der Männer über mäßige oder starke Insomnien klagen. Zwei Studien, die sich mit der Prävalenz von Schlafstörungen in der gesamtdeutschen Bevölkerung beschäftigt haben, nähern sich dem Ergebnis von Ohayon an: Die Studie zur Gesundheit Erwachsener in Deutschland ergab unter 8152 Teilnehmern zwischen 18 und 79 Jahren eine Prävalenz von Schlafproblemen von 5,7\%. Das Insomniesyndrom wurde in der bundesweiten Studie nach den Kriterien von DSM-IV und ICD-10 als Kombination aus Insomniesymptomen mit schlechter Schlafqualität definiert. Frauen waren doppelt so häufig betroffen wie Männer. Signifikante Alterseffekte zeigten sich hingegen nicht 
(Schlack et al. 2013). Mit Hilfe von persönlichen Befragungen ergab sich in einer Teilstudie eines internationalen Projekts zu Schlafstörungen eine Prävalenz von schwerer Insomnie (nach DSM-IV-Kriterien) von 4 \% für die Gesamtbevölkerung Deutschlands (Hajak 2001). Für Menschen über 65 Jahre nennen Studien eine deutlich höhere Prävalenz von Schlafstörung zwischen 20 bis $40 \%$ (Ancoli-Israel 2005).

\subsection{Pharmakologische Therapie bei Schlafstörungen mit Benzodiazepinen und Z-Substanzen}

Schlafstörungen können durch unterschiedliche pharmakologische Therapien behandelt werden. Benzodiazepine und die benzodiazepinähnlichen Z-Substanzen kommen vorwiegend zum Einsatz und gehören zu den klassischen Hypnotika und Sedativa bzw. auch Tranquilizern.

\subsubsection{Exkurs: Historie der Benzodiazepine und Z-Substanzen}

Die Entwicklungsgeschichte der Benzodiazepin-Hypnotika begann in den 1950er Jahren. Der Firma Hoffmann-La Roche gelang im Rahmen einer Forschungsreihe an Heptodiazinen die Synthese der ersten Benzodiazepine in Nutley, New Jersey in den USA. In Tierversuchen mit Mäusen wurde Chlordiazepoxid zunächst auf tranquilisierende und sedative Eigenschaften getestet (Sternbach 1971). Diesen Hypnotika, die zunächst für biologisch inaktiv gehalten worden waren, konnte 1957 eine hypnotische und sedierende Wirkung nachgewiesen werden (Lader 1991). Die ersten Benzodiazepin-Derivate hießen Methaminodiazepoxide oder später auch Chlordiazepoxide. 1960 wurde das Medikament offiziell zugelassen und drei Jahre später folgte die Einführung von Diazepam.

Trotz des Nachweises von Gewöhnungseffekten und Entzugssymptomen bei einer Einnahme in hohen Dosen in 1961 (ein Jahr nach Markteinführung des ersten BenzodiazepinDerivates) stieg der Benzodiazepingebrauch in den 60er und 70er Jahren stark an, gemeinsam mit dem Gebrauch anderer angstlösender und beruhigender Medikamente. Inzwischen ist wissenschaftlich belegt, dass Benzodiazepine verschiedene Nebenwirkungen und besonders bei älteren Menschen Einfluss auf die kognitiven Fähigkeiten haben. Eine Demenzentwicklung durch Benzodiazepine oder Z-Substanzen wird kontrovers diskutiert und kann bisher nicht ausgeschlossen werden (Puustinen et al. 2007; Billioti de Gage et al. 2012; Billioti de Gage et al. 2014; Billioti de Gage et al. 2015; Gray et al. 2016). Benzodiazepine wurden nach Markteinführung in der Gruppe der Hypnosedativa immer häufiger verschrieben und zählen seit 1963 zu den wichtigsten Arzneimittel in der Behandlung von Angststörungen und Neurosen (Hollister et al. 1961; Sternbach 1979; Lader 1991).

Die Benzodiazepin-ähnlichen Z-Substanzen folgten als „Hypnotika der dritten Generation“ in den späten 1970er und Anfang der 1980er Jahre. Sie gewannen zunehmend an Bedeutung, 
während die Verordnung von langwirksamen Benzodiazepinen abnahm. Zu den Z-Substanzen gehören: Zopiclon, Zolpidem und Zaleplon (Glaeske et al. 2013).

Benzodiazepine und Z-Substanzen sind heute feste Bestandteile der Pharmakotherapie. Aufgrund ihres Abhängigkeitspotenzials, der Nebenwirkungen und der Entzugserscheinungen sind Benzodiazepine sowie Z-Substanzen rezeptpflichtig, an klare Indikationen gebunden und nur für die Kurzzeitbehandlung bzw. als Intervalltherapie empfohlen (Schutte-Rodin et al. 2008; DGSM 2009; DGSM 2017; Pinto Jr. et al. 2010; Glaeske et al. 2013).

\subsubsection{Leitliniengerechte Therapie}

Folgende Behandlungen werden durch die S3-Leitlinie (DGSM 2009; DGSM 2017) mit dem Empfehlungsgrad A zur Therapie von Schlafstörungen angeraten:

1. Primär empfehlen sich als erster Therapieversuch kognitiv-verhaltenstherapeutische Methoden, insbesondere auch für ältere Patienten.

2. Eine pharmakologische Therapie mit Benzodiazepinrezeptoragonisten und den neueren Präparaten (Z-Substanzen) soll zweitranging zur Anwendung kommen, wenn die kognitiv-verhaltenstherapeutische Behandlung nicht erfolgreich war. Die medikamentöse Therapie wird kurzzeitig für drei bis vier Wochen empfohlen. Ebenso wird die Behandlung mit sedierenden Antidepressiva (zum Beispiel Mirtazapin) bei der Abwesenheit von Kontraindikation und unter Verlaufskontrollen angeraten.

Für ältere Patienten wird bei Benzodiazepinrezeptoragonisten und sedierenden Antidepressiva auf unerwünschte Arzneimittelwirkungen hingewiesen: Hier wird speziell die erhöhte Sturzneigung genannt, die bei älteren Patienten aufgrund der größeren Frakturgefahr von besonderer Bedeutung sei. Eine der möglichen Alternativen - Melatonin - erreicht nur den Empfehlungsgrad B und wird nicht generell empfohlen. Zu Neuroleptika und Phytotherapeutika, wie zum Beispiel zu Baldrianpräparaten, wird aufgrund der ungenügenden Studienlage nicht geraten (DGSM 2009; DGSM 2017).

Die Empfehlungen zum Beispiel der brasilianischen Leitlinie zur Behandlung von Schlafstörungen unterscheiden sich wenig von denen der deutschen S3-Leitlinie. Auch die brasilianische Leitlinie empfiehlt neben einer kombinierten kognitiven Verhaltenstherapie eine pharmakologische Therapie mit der Z-Substanz Zolpidem als erste Wahl (vergleichbar mit Empfehlungsgrad A). Andere Z-Substanzen und sedierende Antidepressiva werden - wie in der deutschen Leitlinie - ebenfalls befürwortet. Klassische Benzodiazepine werden nicht zur Erstlinientherapie vorgeschlagen. Nachrangig kommen die Benzodiazepine Clonazepam, Mida5zolam und Estazolam in Frage. Alle anderen Präparate dieser Stoffgruppe gelten als nicht empfehlenswert. In der brasilianischen Leitlinie wird außerdem die besondere Verant- 
wortlichkeit bei der Behandlung von älteren Patienten mit Benzodiazepinen betont (Pinto Jr. et al. 2010).

Ebenso wird in der Leitlinie des „Journal of Clinical Sleep Medicine“ zur initialen Intervention bei chronischen primären und sekundären/komorbiden Insomnien die Psycho- und Verhaltenstherapie empfohlen. Eine Therapie sollte demzufolge vor der pharmakologischen Behandlung zumindest einen Interventionsversuch mit Änderung des Verhaltens beinhalten, wenn dies angemessen erscheint und es die individuellen Umstände erlauben. Als ergänzende pharmakologische Therapie wird eine Behandlung mit kurzwirksamen Hypnotika vorgeschlagen, zum Beispiel mit den sogenannten Z-Substanzen. Bei Patienten höheren Alters wird vorrangig zur Psycho- und Verhaltenstherapie geraten (Schutte-Rodin et al. 2008).

Die Leitlinie der European Medicines Agency geht auf die Behandlung von älteren Patienten insbesondere mit dem Hinweis ein, dass bei der Therapie der veränderte Stoffwechsel und die andere Schlafarchitektur beachtet werden müssten. Die Wahl der therapeutischen Dosis solle neben der Wirksamkeit besonders auch das Nebenwirkungsspektrum sowie die Ergebnisse spezieller Studien über die ältere Altersgruppe berücksichtigen (European Medicines Agency 2011).

\subsubsection{Empfehlungen für ältere Patienten laut PRISCUS-Liste}

In der sogenannten PRISCUS-Liste (priscus, lat.: alt, altehrwürdig) von 2010 werden potenziell inadäquate Medikamente aufgeführt, die aufgrund von vermehrten unerwünschten Arzneimittelwirkungen oder erhöhten Risiken für ältere Patienten nicht geeignet sind (Holt et al. 2010). Neben der reinen Auflistung dieser Arzneimittel und der Begründung für ihre potenzielle Untauglichkeit werden Alternativempfehlungen gegeben. Die PRISCUS-Liste beinhaltet 83 Arzneistoffe aus 18 Arzneistoffklassen; unter der Kategorie „Hypnotika, Sedativa“ werden speziell Benzodiazepine und Z-Substanzen aufgeführt. Gruppenübergreifend werden diese Substanzen als potenziell inadäquat für ältere Patienten eingestuft, da sie durch ihre muskelrelaxierende Wirkung mit einem erhöhten Sturzrisiko und möglichen Knochenbrüchen einhergehen. Zusätzlich können durch die sedative Wirkung und die dadurch eingeschränkte Kognition geistige Beeinträchtigungen auftreten. Aus diesem Grund wird besonders von der Behandlung mit langwirksamen Benzodiazepinen abgeraten und stattdessen der Einsatz kurzwirksamer Benzodiazepine oder Z-Substanzen empfohlen. Allerdings gelten auch letztere als potenziell gefährdend, so dass nach Möglichkeit alternative Schlafmittel (wie Baldrian) oder sedierende Antidepressiva (wie Mirtazapin) zum Einsatz kommen sollten, wenn eine medikamentöse Therapie definitiv notwendig ist. Als nicht-medikamentöse Behandlungsoption werden zum Beispiel Maßnahmen der Schlafhygiene angeraten. 


\subsubsection{Wirkungen und Anwendungsgebiete}

Benzodiazepine und Z-Substanzen haben unterschiedliche therapeutische Effekte. Je nach Wirkstoff sind sie angstlösend, antikonvulsiv, muskelrelaxierend, hypnotisch und sedativ. Als Schlafmittel wirken sie schlafanstoßend und helfen beim Durchschlafen (Estler und Schmidt 2007; Glaeske et al. 2013). Anwendung finden sie sowohl im ambulanten als auch im stationären Bereich. Bei Schlafstörungen und Unruhe zeigen sie als typische Wirkungen Muskelrelaxation, Antikonvulsion und Sedierung. Bei Insomnien und Angst werden in der hausärztlichen Versorgung insbesondere Z-Substanzen und kurzwirksame Benzodiazepine verschrieben (Rote Liste 2017). Beide wirken im Zentralnervensystem an GammaAminobuttersäure(GABA)-Rezeptoren agonisierend und zentral beruhigend. Aufgrund dieser sedativen Wirkung werden Benzodiazepine zur Prämedikation in der Anästhesie, aber auch in der Neurologie und Psychiatrie eingesetzt. Die beschriebenen Wirkeigenschaften machen sie dort bei großer therapeutischer Breite und schnellem Effekt zu wichtigen Medikamenten der Akutbehandlung von Psychosen oder schweren Depressionen (Rosenbaum 2005; Estler und Schmidt 2007; Rote Liste 2017).

\subsubsection{Pharmakologie von Benzodiazepinen und Z-Substanzen bei älteren Patienten}

Menschen im fortgeschrittenen Alter sind anfälliger für potenzielle Nebenwirkungen von Benzodiazepinen und Z-Substanzen aufgrund einer sich stetig verändernden Stoffwechsellage des Körpers. Im höheren Alter sinkt die Verteilungs- und Eliminationsfähigkeit von Medikamenten im Körper und potenzielle unerwünschte Arzneimittelwirkungen treten häufiger auf (Woodward 1999; Bogunovic und Greenfield 2004). Veränderungen der Pharmakokinetik aufgrund von Funktionseinschränkungen der inneren Organe im Alter stellen besondere Anforderungen an die Anpassung der Dosis von Arzneimitteln. Gleichzeitig haben individuelle Unterschiede der Physiologie, Komorbiditäten sowie psychische Faktoren Einfluss auf die pharmakokinetischen Prozesse, die möglicherweise altersunabhängig sind, sich aber im höheren Alter häufig summieren (Cusack 2004; Ancoli-Israel 2005).

\section{Benzodiazepine}

Bei der Einnahme von Benzodiazepinen durch ältere Menschen finden sich pharmakokinetische Veränderungen in der Länge der Halbwertszeit. Die Eliminationszeit verlängert sich durch eine verschlechterte Leber- und Nierenfunktion bei Patienten höheren Alters deutlich. Je nach Benzodiazepin kann die Halbwertszeit bis zu über 100 Stunden betragen und es kann zu Akkumulationen der Einzeldosen kommen (Woodward 1999; Riss et al. 2008).

Insbesondere für langwirksame Benzodiazepine ist ein sogenannter hangover-Effekt in der Fachliteratur beschrieben. Durch einen verlangsamten Abbau der Medikamente kommt es zu einer Akkumulation der Restdosen und Abbauprodukte bei der mehrmaligen Benzodiazepineinnahme. Diese führt zu einer andauernden Wirkung über die Ruhezeit in der Nacht hinaus 
bis zum nächsten Morgen und länger - mit der Folge einer verlängerter Reaktionszeit und Abgeschlagenheit, verstärkt bei älteren Patienten (Cook et al. 1983).

Die veränderte Pharmakodynamik älterer Menschen führt bei der Einnahme von Benzodiazepinen dazu, dass schon mit einer geringen Menge eine definierte Sedierung erzielt wird. Bei gleicher Dosierung kommt es zu höheren Konzentrationen des Medikaments im Blutplasma. Diese können zu einer länger andauernden Sedierung führen (Woodward 1999).

\section{Z-Substanzen}

Die Pharmakokinetik und Pharmakodynamik von Z-Substanzen unterscheiden sich von denen der Benzodiazepinen, da Z-Substanzen einen anderen Angriffspunkt am GABARezeptor im Gehirn haben. In experimentellen Studien konnte aber auch bei Einnahme von Z-Substanzen ein Anstieg der Plasmahalbwertszeit mit steigendem Alter nachgewiesen werden (Gaillot et al. 1983), so dass es zu einem verzögerten Abbau des Arzneimittels im Körper und dadurch zu einer Verlängerung der Wirkungen und Nebenwirkungen kommt. Mehrere Z-Substanzen sind von dem CYP-Metabolismus der Leber abhängig. Einschränkungen dieser Abbauprozesse durch den natürlichen Altersprozess der Leber und Niere können zum einen zu einer erhöhten Bioverfügbarkeit der Arzneimittel und gleichzeitig zu einem längeren Verbleib im Körper führen. Dies kann einen Überhang der Wirkung bis zu acht Stunden nach Dosisgabe und einer verlängerten Einschränkung der kognitiven Fähigkeiten zur Folge haben (Dolder et al. 2007).

\subsubsection{Nebenwirkungen durch die Einnahme von Benzodiazepinen und Z-Substanzen}

Ein breites Nebenwirkungsspektrum durch die Einnahme von Benzodiazepinen und ZSubstanzen ist bekannt. Insbesondere im höheren Alter können Auswirkungen auf das Zentralnervensystem und die Lokomotorik durch bereits bestehende altersbedingte Einschränkungen ernsthafte Risiken in sich bergen. In einer kanadischen Meta-Analyse von 24 Studien zur Beurteilung von Nutzen und Risiken der Einnahme von sedierenden Hypnotika durch ältere Patienten konnten folgende negative Arzneimittelwirkungen - im Vergleich zur Einnahme eines Placebos - nachgewiesen werden: Unerwünschte kognitive Erscheinungen waren fast 5-mal häufiger, Tagesmüdigkeit 3,8-mal und psychomotorische Einschränkungen im Durchschnitt 2,6-mal häufiger. Dieser Anstieg der Risiken könnte für ältere Patienten gegenüber den Einnahmenutzen überwiegen (Glass et al. 2005).

\section{Benzodiazepine}

Das Nebenwirkungsspektrum von Benzodiazepinen verändert sich mit einem höheren Alter der Patienten; vor allem sind es Schwindel, Konzentrationsstörungen, Gangunsicherheit, Stürze, Gedächtnisprobleme und Demenzerkrankungen. Gangunsicherheit bzw. Stürze und damit verbundene Hüftfrakturen gehören zu den schwersten Nebenwirkungen. Mehrere Stu- 
dien haben ein erhöhtes Risiko für Hüftfrakturen bei Einnahme von Benzodiazepinen im höheren Alter nachgewiesen (Ray et al. 1989; Wang et al. 2001a; Wagner et al. 2004). Das erhöhte Sturzrisiko ist laut einer französischen Querschnittsstudie assoziiert mit den zunehmenden Komorbiditäten im höheren Alter. Bei einer Komorbiditätsrate von bis zu über 50 \% der älteren Bevölkerung, lag das Sturzrisiko zwischen 10,8 \% und 13,4 \% (Bénard-Laribière et al. 2016). Das Ergebnis der integrierten Fall-Kontroll-Studie von Ray et al. (1989) beschrieb ein um den Faktor 1,7 erhöhtes Risiko für Hüftfrakturen bei Einnahme von Benzodiazepinen mit einer langen Halbwertszeit. Im Vergleich dazu ergab sich eine Risikoerhöhung um 1,1 bei kurzwirksamen Benzodiazepinen. Eine weitere Studie hat ein erhöhtes Sturzrisiko insbesondere für das langwirksame Diazepam bestätigt (Ballokova et al. 2014). Erstaunlicherweise haben kanadische Studien belegt, dass Patienten mit vorbestehenden Risiken für Stürze mit erhöhter Wahrscheinlichkeit ein Benzodiazepin neu verschrieben bekommen (Bartlett et al. 2009). In einer aktuellen Studie wird eine erhöhte Verletzungsgefahr für ältere Patienten durch Stürze nach Einnahme von Benzodiazepinen oder Z-Substanzen bestätigt, die dann zu vermehrten Krankenhauseinweisungen führen (Yu et al. 2017).

Neben der Sturzgefahr lösen Benzodiazepine laut einer finnischen Studie als weitere zentrale Nebenwirkungen auch Schwindel, Schlaflosigkeit, Müdigkeit am Morgen und Depression aus (Puustinen et al. 2007). Benzodiazepine sollen außerdem das Zentralnervensystem ähnlich einer diagnostizierten Demenz beeinflussen, da eine Akkumulation von Benzodiazepinen im Blut Auswirkungen auf die Gedächtnisleistung haben kann: So konnten drei französische Studien einen Zusammenhang zwischen der Einnahme von Benzodiazepinen und dem Risiko für die Entwicklung einer Demenzerkrankung im höheren Lebensalter, speziell auch der Alzheimer-Demenz, nachweisen (Lagnaoui et al. 2002; Billioti de Gage et al. 2012; Billioti de Gage et al. 2014; Billioti de Gage et al. 2015). Billioti de Gage et al. zeigten diese Assoziation für den Langzeitgebrauch von Benzodiazepinen bei älteren Menschen, während Lagnaoui et al. zusätzlich auch den Unterschied zwischen aktuellem und früherem Gebrauch (Einnahmeende innerhalb der letzten zwei bis drei Jahre) und dem Einfluss auf eine sich entwickelnde Demenzerkrankung untersuchten. In einer integrierten Fall-Kontroll-Studie zeigte sich eine Risikosteigerung um den Faktor 1,7 für den mindestens einmaligen Gebrauch. Eine frühere Einnahme lässt das Risiko für die Entwicklung einer Demenzerkrankung um den Faktor 2,3 steigen.

Der Zusammenhang zwischen Benzodiazepineinnahme und dem Rückgang der kognitiven Leistung ist weiterhin umstritten und wird kontrovers diskutiert. So konnte zum Beispiel in einer Drei-Städte-Studie in Frankreich bei 969 Benzodiazepin-Verwendern im Vergleich zu 4426 Nicht-Verwendern im Alter von 65 Jahren und älter eine schwächere geistige Leistung festgestellt werden, aber keine signifikante Beschleunigung der kognitiven Einschränkungen 
(Mura et al. 2013). Ebenso konnte eine aktuelle Studie aus den USA keinen kausalen Zusammenhang zwischen einer Benzodiazepineinnahme und einer Demenzentwicklung nachweisen (Gray et al. 2016).

\section{Z-Substanzen}

Z-Substanzen werden im Vergleich zu Benzodiazepinen zunehmend häufiger verschrieben und als sicherer eingeschätzt. In einer Studie bewerteten Hausärzte und Apotheker Z-Substanzen bezüglich der Effektivität und Unbedenklichkeit als vorteilhafter gegenüber Benzodiazepinen (Hoffmann 2013). Entsprechendes ergab auch eine Befragung von britischen Hausärzten: Die Mehrheit schätzte Z-Substanzen als effektiver und nebenwirkungsärmer ein. Zusätzlich wurden Z-Substanzen speziell in der Behandlung von älteren Patienten als risikoärmer bewertet (Siriwardena et al. 2006). Für diese Beurteilung von Z-Substanzen gibt es wenig Evidenz. Z-Substanzen werden in Sicherheit und Nebenwirkungen als vergleichbar zu Benzodiazepinen eingestuft und sollen mit derselben Vorsicht verordnet werden (Dündar et al. 2004; NICE-National Institute of Clinical Excellence 2004; Siriwardena et al. 2008; Siriwardena et al. 2012; Gunja 2013).

Z-Substanzen besitzen in Abhängigkeit vom Präparat einen individuellen Angriffspunkt im ZNS und eine eigene Pharmakokinetik. Eine Studie aus den 1990er Jahren aus Großbritannien hatte die Auswirkungen von Zolpidem bei älteren Patienten hinsichtlich des hangoverEffektes am nächsten Morgen und der subjektiven Verbesserung des Schlafes untersucht. Das Ergebnis ergab eine (subjektiv bewertet) bessere Schlafqualität ohne nachweisbare Beeinträchtigungen der Leistungsfähigkeit am nächsten Morgen und ohne messbare Gewöhnungseffekte oder negative Auswirkungen auf die kognitive Leistung beim Lösen vorgegebener Aufgaben (Fairweather et al. 1992). Zunächst gingen die Empfehlungen dahin, ZSubstanzen mit der gleichen Sorgfalt und Vorsicht einzusetzen wie ein Benzodiazepinpräparat, da die Studienlage über diese neuen Arzneimittel noch nicht ausreichte. Da ZSubstanzen an denselben Rezeptoren wie die Benzodiazepine binden, wurde vermutet, dass sich in Langzeitstudien und intensiver klinischen Anwendung ähnliche Wirkungen und $\mathrm{Ne}$ benwirkungen zeigen würden (Woodward 1999). Mittlerweile haben mehrere Studien ein vergleichbares Spektrum an Nebenwirkungen von Z-Substanzen und Benzodiazepinen bestätigt. In einer Meta-Analyse zählten Schläfrigkeit und Schwindelgefühl zu den häufigsten unerwünschten Arzneimittelwirkungen. Seltener hingegen wurden Erregungszustände und die Verschlechterung von Schlafstörungen genannt (Dolder et al. 2007). Für Zopiclon wurden typische Einschränkungen der kognitiven und psychomotorischen Leistungsfähigkeit sowie des Erinnerungsvermögens und des Gleichgewichts spezifiziert (Mets et al. 2011). Auswirkungen auf die psychomotorischen und kognitiven Fähigkeiten waren bei Z-Substanzen im 
Vergleich zu Benzodiazepinen seltener (Fairweather et al. 1992; Woodward 1999; Dolder et al. 2007).

Nachdrücklich wurde auf die Auswirkungen für ältere Menschen eingegangen: Mehrere Studien zeigten eine erhöhte Gefahr für Stürze und Frakturen speziell bei Menschen im Alter von 65 Jahren oder älter. Das Risiko war insbesondere für Zolpidem mit dem von Benzodiazepinen (ausdrücklich Diazepam) vergleichbar (Wang et al. 2001b; Finkle et al. 2011; Diem et al. 2014; Yu et al. 2017). Andere Nebenwirkungen waren die Entwicklung von Wahnvorstellungen, Halluzinationen und eines Deliriums (Dolder et al. 2007). Insbesondere wurden Beeinträchtigungen der Sicherheit beim Fahren eines Kraftfahrzeugs für die Z-Substanzen Zopiclon und Zolpidem nachgewiesen. Dies kann bei möglichen altersbedingten geistigen Einschränkungen, die eine Fahrsicherheit bereits beeinträchtigen können, zu Unfällen führen (Vermeeren et al. 1998; Leufkens et al. 2009; Mets et al. 2011; Gunja 2013; Vermeeren et al. 2014). Gunja weist in einem Review von 2013 darauf hin, dass eine Risiko-NutzenAbwägung in Hinsicht auf das erhöhte Risiko für Stürze und (Auto-) Unfälle eine Behandlung von älteren Patienten mit Z-Substanzen fraglich werden lässt.

\subsubsection{Toleranz- und Abhängigkeitspotenzial von Benzodiazepinen und Z-Substanzen}

Benzodiazepine

Eine Toleranzentwicklung durch den Dauergebrauch von Benzodiazepinen ist nachgewiesen, allerdings nicht gänzlich verstanden. Benzodiazepine wirken im Zentralnervensystem an speziellen Rezeptoren. Bewiesen werden konnte bisher eine „Herunterregulierung“ (down regulation) dieser Benzodiazepin-Rezeptoren und eine verminderte Funktion der GABARezeptoren bei längerer Einnahme, die für eine zunehmende Gewöhnung an die Wirkung verantwortlich scheint (Miller et al. 1988). Bei den meisten der verwendeten Medikamente konnte ein Toleranzeffekt nachgewiesen werden. Bereits 1984 konnte eine Gewöhnung an Diazepam für alle Altersgruppen gezeigt werden, allerdings ohne einen Unterschied zwischen älteren und jüngeren Patienten belegen zu können (Cook et al. 1984). Diese Toleranzentwicklung und daraus resultierende höhere Dosen von Benzodiazepinen können das Risiko einer Abhängigkeit in sich bergen und durch Akkumulation zu vermehrten unerwünschten Arzneimittelwirkungen führen.

Das Abhängigkeitspotenzial von Benzodiazepinen konnte sowohl in mehreren Tierversuchen als auch in Studien mit menschlichen Versuchsteilnehmern nachgewiesen werden (Petursson und Lader 1981). Ein höheres Alter ist kein Risikofaktor für eine Abhängigkeit von Benzodiazepinen, aber eine häufiger vorkommende Langzeiteinnahme lässt ein erhöhtes Vorkommen von Abhängigkeit unter der älteren Bevölkerung annehmen. Bei der dauerhaften Einnahme von Benzodiazepinen kann es zu Missbrauch kommen. Toleranzentwicklung und Dosissteigerung (abweichend von der verschriebenen therapeutischen Dosierung) ebenen 
Suchtverhalten (O'Brien 2005). Empfohlen wird, eine - über die akute Notwendigkeit hinaus - andauernde Einnahme zu vermeiden, da diese den Toleranzeffekt und das Abhängigkeitsrisiko steigere (Woodward 1999; Barker et al. 2004; O’Brien 2005; Rosenbaum 2005).

\section{Z-Substanzen}

Die Toleranzentwicklung bei Z-Substanzen wurde aktuell für Zolpidem im Vergleich zu Diazepam im Tierversuch verglichen. So konnte bei Mäusen eine Gewöhnung bei subchronischer Gabe für Zolpidem bestätigt werden (Wright et al. 2014). In einem deutschen Review von Hajak et al. (2003) wurden retrospektiv Fallstudien und epidemiologische Daten der am häufigsten verschriebenen Z-Substanzen Zolpidem und Zopiclon zusammengefasst und bewertet. Insgesamt konnte eine wesentlich geringere Inzidenz an dokumentierten Fällen von Abhängigkeit für beide Z-Substanzen im Vergleich zu Benzodiazepinen erfasst werden. Dennoch konnte ein erhöhtes Abhängigkeitsrisiko für Patienten mit einer Abusus- oder Abhängigkeitsvorgeschichte festgestellt werden.

\subsubsection{Entzugserscheinungen nach der Einnahme von Benzodiazepinen und Z- Substanzen}

Benzodiazepine

In Studien konnten nach Abbruch der Einnahme bei Patienten, die Benzodiazepine regelmäBig und über einen längeren Zeitraum eingenommen hatten, Entzugserscheinungen nachgewiesen werden. Bereits ein Jahr nach der Markteinführung des ersten Präparats aus der Familie der Benzodiazepine wurden 1961 sowohl Nebenwirkungen als auch Entzugserscheinungen beobachtet. Patienten beschrieben Depressionen, Verschlechterungen von Psychosen, Unruhe, Schlaf- und Appetitlosigkeit sowie Übelkeit im Zeitraum von zwei bis acht Nächten nach abruptem Absetzen von dem zuerst zugelassenen Benzodiazepinpräparat Chlordiazepoxid (Hollister et al. 1961). Die typischen Entzugssymptome variieren in den Studien von Angstgefühlen und Dysphorie bis zu starken Veränderungen der Wahrnehmung und der Gefühle (Hallstrom und Lader 1981; Petursson und Lader 1981).

Bei älteren Patienten wurden andere Entzugssymptome beobachtet als bei jüngeren. Bei älteren Menschen waren insbesondere Verwirrung und Desorientierung - zum Teil einhergehend mit Halluzinationen - die vorherrschenden Symptome einer Entwöhnung nach abruptem Beenden der Einnahme. Auf der anderen Seite entwickelten ältere Patienten nicht so häufig die sonst typischen Entzugserscheinungen wie Angstgefühle, Wahrnehmungsstörungen oder Schlaflosigkeit (Hallstrom und Lader 1981; Petursson und Lader 1981; Foy et al. 1986; O’Brien 2005; Authier et al. 2009). 


\section{Z-Substanzen}

In einer Tierversuchsreihe mit Mäusen konnten Entwöhnungseffekte nach Absetzen von Zolpidem nach einer Woche der täglichen Injektionen festgestellt werden. Die Mäuse zeigten einen Tag nach Unterbrechung der Zolpidemgabe ein angstähnliches Verhalten (Wright et al. 2014). Andere Entzugserscheinungen, wie zum Beispiel die einer rebound-Insomnie nach Absetzen von Z-Substanzen, konnten bisher nicht nachgewiesen werden (Dolder et al. 2007). In der Literatur wird mehrmals auf die limitierte Studienlage über Z-Substanzen und ihre Auswirkungen auf den menschlichen Organismus hingewiesen und zum vorsichtigen Umgang - analog zu dem mit Benzodiazepinen - geraten.

\subsubsection{Häufigkeit der Einnahme von Benzodiazepinen und Z-Substanzen}

Laut Arzneimittelverordnungsreport von 2015 wurden im Jahr 2014 (im Studienzeitraum) Hypnotika und Sedativa im Umfang von 104 Millionen definierten Tagesdosen (DDD = defined daily doses) zu Lasten der gesetzlichen Krankenkassen verschrieben. Im letzten Report über 2016 waren es immer noch 97 Millionen DDD (Schwabe und Paffrath 2017). Seit Jahren gibt es einen stetigen Rückgang, so waren es 1992 mit 476 Millionen DDD rund 80 \% mehr Verschreibungen gewesen. Benzodiazepine waren mit 23 Millionen DDD und ZSubstanzen Zopiclon sowie Zolpidem mit 74 Millionen DDD im Jahr 2012 die am häufigsten verschriebenen Arzneimittel dieser Substanzgruppe. Besonders die Verordnung von Benzodiazepinen zeigte gemäß Report seit Jahren einen starken Rückgang. Zehn Jahre zuvor waren noch 58 Millionen DDD verschrieben worden, 2011 waren es noch 34 Millionen DDD und im aktuellen Report über 2015 waren es nur noch 18 Millionen DDD. Die Verschreibung der Z-Substanzen blieb zu den letzten drei Vorjahren relativ konstant und liegt aktuell bei 70 Millionen DDD, sie lag aber fünf Jahre zuvor mit 81 Millionen DDD höher. Pflanzliche Präparate werden seit Jahren im Bereich von 3 bis 4 Millionen DDD verschrieben, so auch in 2014, und sind rezeptfrei zu erhalten. In 2016 gab es einen leichten Anstieg auf 5 Millionen DDD (Schwabe und Paffrath 2015; Schwabe und Paffrath 2017). Es ist mittlerweile bekannt, dass in Deutschland Benzodiazepinhypnotika und Z-Substanzen in der ambulanten Versorgung in 43-58 \% der Fälle je nach Substanz über ein Privatrezept verschrieben werden (Hoffmann und Glaeske 2014). So könnten die Daten des Arzneimittelverordnungsreports die tatsächlichen Verschreibungen von Benzodiazepinen und Z-Substanzen um fast die Hälfte unterschätzen.

International werden die Einnahmen von Hypnotika und Sedativa in der ambulanten Versorgung zumeist in Jahres-Prävalenzen berechnet und bewegen sich in einem breiten Intervall von 8 bis $40 \%$ - insbesondere wenn man nach dem Alter differenziert:

In einer repräsentativen Schweizer Querschnittsstudie hatten innerhalb eines halben Jahres 9,1\% der Teilnehmer mindestens ein Benzodiazepin verordnet bekommen (Petitjean et al. 
2007). Eine Studie aus den Niederlanden bezieht sich auf ganze Jahre. Hier lagen die Prävalenzen des Benzodiazepingebrauchs mit 7,8\% bzw. 7,9\% für die Jahre 1992 und 2002 unter $10 \%$ (Sonnenberg et al. 2012). Aktuelle Daten aus den USA geben mit 5,6\% eine noch niedrigere ambulante Verordnung von Benzodiazepinen an, die mit höherem Alter auch nur auf 6,6\% ansteigt (Maust et al. 2016). Höhere Verordnungen ergaben sich laut einer französischen Querschnittsstudie aus Daten der nationalen französischen Krankenversicherung. Hier hatten 2013 13,8 \% der Versicherten ambulant mindestens ein Benzodiazepinpräparat erhalten. Die Prävalenz war höher bei Frauen sowie bei Menschen über 65 Jahre (Bénard-Laribière et al. 2016).

In der Altersgruppe über 65 Jahre liegt die Einnahmeprävalenz von Schlaf- und Beruhigungsmitteln in einer Spanne von $10 \%$ bis $40 \%$. Die oben erwähnte französische Querschnittsstudie ergab eine Einnahmerate von Benzodiazepinen von 35,2 \% bei älteren Frauen und 21,6 \% bei älteren Männern. Menschen im Alter von 80 Jahren und darüber hatten mit $39,3 \%$ die höchste Prävalenz (Bénard-Laribière et al. 2016). In sechs australischen Altersresidenzen nahmen 25,1\% der älteren Bewohner regelmäßig Benzodiazepine ein (Chen et al. 2016). Etwas niedriger war die Verschreibung von Benzodiazepinen in der Schweizer Studie von Petitjean et al. (2007) mit 20,9 \% für ältere Patienten, aber gleichzeitig etwa doppelt so hoch wie die der Gesamtpopulation. Im deutschen Barmer GEK Arzneimittelreport von 2010/2011 lag die Verordnungsprävalenz bei 14,9 \% (Glaeske et al. 2013). Eine aktuelle schottische Studie schätzt die Verschreibung von Benzodiazepinen oder Z-Substanzen auf $12,1 \%$ bei älteren Menschen (Johnson et al. 2016). Geringer schätzte eine ältere Studie aus den USA die Prävalenz: 9,9\% der älteren Patienten hatten mindestens ein Benzodiazepinpräparat eingenommen und über ein Drittel $(39,2 \%)$ nahm zusätzlich noch freiverkäufliche Schlafmittel ein (Gleason et al. 1998).

Studien zur Prävalenz von Schlaf- und Beruhigungsmitteln in der stationären Versorgung ergeben Einnahme-Prävalenzen zwischen $20 \%$ und $52 \%$, im Regelfall auf die Aufenthaltsdauer berechnet:

So erhielten in einer schon älteren Studie $21 \%$ der Patienten eines britischen Lehrkrankenhauses Hypnotika im Rahmen ihres stationären Aufenthalts. Bei $80 \%$ dieser Verschreibungen handelte es sich um Benzodiazepine (Edwards et al. 1991). Ähnlich hoch war die Frequenz der Verordnung in einem japanischen Universitätsklinikum. Hier lag die Prävalenz der stationären Patienten bei 19,9\%, bei den älteren Patienten sogar bei ca. $23 \%$ (Nakao et al. 2009). Deutlich höher war die Einnahme von Hypnotika in einer kanadischen Studie mit $34 \%$ aller Patienten während des Aufenthalts in einer Universitätsklinik (O'Reilly und Rusnak 1990). Vergleichbares ergab eine US-amerikanische Studie: Über $40 \%$ der Patienten über 65 Jahre erhielten ein Benzodiazepin während des stationären Aufenthalts (Zisselman et al. 
1994). Der Anteil der Verordnungen in drei niederländischen Krankenhäusern war mit fast der Hälfte der Patienten (47\%) sogar noch höher. Diese Studie hatte die generelle Schlafmedikation der Patienten während ihres stationären Aufenthalts erhoben (Halfens et al. 1991). In einer neueren belgischen Studie lag die Verschreibungsprävalenz in einem ähnlich hohen Bereich: 43,3 \% der stationären Patienten hatten laut der Patientenakte Hypnosedativa verordnet bekommen. Dabei handelte es sich mehrheitlich um Benzodiazepinpräparate. Die Häufigkeit stieg an mit dem Alter der Patienten (Somers et al. 2011). In einer vorherigen Studie im selben Genter Universitätskrankenhaus lag die Verordnungshäufigkeit sogar bei 45,2 \% der Patienten während des stationären Aufenthalts (Warie et al. 2003). Die meisten stationären Verordnungen von Benzodiazepinen erhielten Patienten laut einer älteren australischen Studie. Hier nahmen 52 \% während ihres Krankenhausaufenthalts Benzodiazepine ein (Noble et al. 1993).

Weibliches Geschlecht und höheres Alter waren in fast allen Studien Prädiktoren für eine vermehrte Einnahme von Hypnotika. Nur Studien aus den 1990er Jahren konnten keinen Geschlechtseffekt und eine ebenfalls ältere Studie auch keinen Alterseffekt bestätigen (O’Reilly und Rusnak 1990; Noble et al. 1993; Zisselman et al. 1994).

\subsection{Die Patientenperspektive auf den Gebrauch von Benzodiazepinen und Z- Substanzen}

Es liegen nur wenige Studien zu den subjektiven Erfahrungen von Patienten unter der Einnahme von Schlaf- und Beruhigungsmitteln, insbesondere von Benzodiazepinen und ZSubstanzen, vor: Zwei Studien aus den 1990er Jahren in Großbritannien haben die Langzeiteinnahme von Benzodiazepinen und die Einschätzung der Patienten untersucht. Danach handelt es sich bei den Patienten, die Benzodiazepine einnahmen, um eine inhomogene Patientengruppe. Die Studien widersprachen also dem in den Medien verbreitetem Stereotyp einer Frau im mittleren Alter, die passiv und dauerhaft ein potenziell abhängig machendes Medikament einnimmt. Mehr als die Hälfte der Patienten äußerte stattdessen den Wunsch, das Benzodiazepin-Medikament wieder abzusetzen. Sie fühlten sich außerdem unzureichend durch ihre Ärzte beraten und über die Einnahme respektive das Absetzen des Medikamentes ungenügend informiert (King et al. 1990; Barter und Cormack 1996).

In der Studie von King et al. (1990) berichteten zudem 16 \% der Befragten von unerwünschten Arzneimittelwirkungen. In den meisten Fällen handelte es sich um Beeinträchtigungen des Gedächtnisses oder der Konzentration sowie um hangover-Effekte. 80 \% nahmen durch den Gebrauch der Medikamente Verbesserungen wahr; am häufigsten besserten sich Schlafprobleme und innere Unruhe. Fast $60 \%$ der Patienten hatten schon einmal versucht, 
die Benzodiazepine abzusetzen, aber meist wegen weiterhin bestehender Beschwerden die Einnahme doch fortgesetzt.

Eine qualitative Studie aus den USA hat ältere Patienten zur Einnahme und zum Langzeitgebrauch von Benzodiazepinen befragt. Die Patienten im Alter zwischen 61 und 95 Jahren nahmen Benzodiazepine vor allem wegen ihrer Schlaf- oder Angststörungen ein; viele beschrieben Stressprobleme unterschiedlicher Art. Die Einnahme der Arzneimittel sei das Einzige, das ihnen helfen würde bzw. hätten sie bereits andere Mittel, die weniger wirkungsvoll gewesen seien, probiert. Eine körperliche Abhängigkeit und/oder Nebenwirkungen wurden von der Mehrheit verneint oder bagatellisiert. Psychische Abhängigkeit zeigte sich in der starken Abneigung und dem robusten Widerstand gegen ein Ausschleichen der Medikamente oder den Abbruch der Therapie - begründet durch die Sorge um das Leiden ohne die Einnahme von Benzodiazepinen (Cook et al. 2007).

In einer weiteren qualitativen Studie wurden belgische Patienten nach der ersten Verschreibung von Benzodiazepinen in ihren Hausarztpraxen befragt. Im Interview beschrieben die Patienten ein Dilemma zwischen dem Wunsch nach einer erfolgreichen medikamentösen Behandlung und den negativen Assoziationen mit Benzodiazepinen. Sie rechtfertigten die Einnahme mit dem Hinweis auf ihre Probleme und einem angeblich minimalen Gebrauch der Medikamente. Das Wissen über Benzodiazepine war unter den Teilnehmern gering, und die Ärzte waren selten um Aufklärung gebeten worden. Dennoch sei den Patienten das Risiko einer psychischen Abhängigkeit und Konditionierung bewusst gewesen. Der Wunsch nach nicht-pharmakologischen Alternativen sei nicht geäußert worden, aber hätte es entsprechende Angebote gegeben, wären diese auf positive Reaktionen getroffen (Anthierens et al. 2007).

Eine britische Studie diente der hier durchgeführten Studie als Orientierung und Vergleich. Dort erhielten 1600 Patienten aus allgemeinärztlichen Praxen einen selbsterklärenden Fragebogen; 935 Fragebögen wurden ausgewertet. Ziel der Studie war es, die Erfahrungen von Patienten mit der Einnahme von Benzodiazepinen und Z-Substanzen zu erfassen und zu vergleichen. 75,4\% von innen hatten ein Medikament gegen Insomnie erhalten. 41,8 \% dieser Patienten berichteten, dass sie mindestens eine Nebenwirkung wahrgenommen hatten; 18,6 \% äußerten den Wunsch, das Medikament abzusetzen und 48,5\% hatten einen Versuch unternommen, die Einnahme zu beenden. Patienten, die Z-Substanzen eingenommen hatten, wünschten häufiger ein Ende der Einnahme und hatten im Vergleich zu den Patienten mit Benzodiazepineinnahme ebenfalls häufiger einen Absetzversuch unternommen. Mit Blick auf wahrgenommene Nebenwirkungen und Verbesserungen unterschieden sich die Patientengruppen nicht. Die häufigste negative Arzneimittelwirkung aus Sicht der Patienten war Tagesmüdigkeit (Siriwardena et al. 2008). 
Zusammenfassend kann man feststellen, dass zahlreiche Studien in den letzten Jahrzehnten auf die besondere Problematik der Behandlung von Schlafstörungen im Alter hingewiesen haben. Die Schlaf- und Beruhigungsmittel aus der Stoffgruppe der Benzodiazepine und ZSubstanzen werden weiterhin gerade älteren Patienten sehr häufig verordnet. In der besonderen Situation während eines Krankenhausaufenthalts können Schlafprobleme, Unruhe und Angstgefühle auftreten, die nicht selten mit Schlaf- und Beruhigungsmitteln behandelt werden. Die Behandlung älterer Patienten ist dabei eine besondere Herausforderung für Pflegekräfte und Ärzte. Allerdings sind bislang die subjektiven Erfahrungen, die Patienten mit der Einnahme von Schlaf- und Beruhigungsmitteln machen, unzureichend untersucht. Es fehlen - besonders in Deutschland - quantitative Daten zur Häufigkeit der stationären Einnahme und darüber, was Patienten über ihre Schlafmedikation wissen, wie sie diese beurteilen und ob sie die Einnahme nach Entlassung fortführen wollen. Insbesondere über die Sicht älterer Patienten ist wenig bekannt. 


\section{Fragestellungen und Hypothesen}

Diese Studie sollte die Einnahme von Schlaf- und Beruhigungsmitteln durch ältere Patienten im Krankenhaus und zu Hause untersuchen. Fünf Fragen stehen im Vordergrund der Studie:

(1) Wie viele Patienten nehmen Schlaf- oder Beruhigungsmittel im Krankenhaus ein?

(2) Stimmen die Angaben der Patienten mit denen der Krankenhausdokumentation überein?

(3) Welche Rolle spielen dabei Vorerfahrungen mit diesen Medikamenten?

(4) Welche Vor- und Nachteile sehen die Patienten für sich in der Einnahme von Schlaf- und Beruhigungsmitteln?

(5) Führt die Einnahme dieser Medikamente zum Wunsch nach einer Weiterverordnung im Anschluss an den Krankenhausaufenthalt?

Mit Bezug auf diese Leitfragen wurden folgende Hypothesen aufgestellt:

1. Vergleichsweise viele Patienten erhalten im Krankenhaus ein Hypnotikum als Schlafoder Beruhigungsmittel.

2. Frauen nehmen - zu Hause wie auch im Krankenhaus - häufiger Schlaf- oder Beruhigungsmittel ein als Männer.

3. Viele Patienten wissen nicht, ob und welche spezifischen Medikamente sie im Krankenhaus bei Schlafproblemen erhalten.

4. Patienten nehmen häufig Verbesserungen wahr, selten hingegen einnahmebedingte Nebenwirkungen: Verbesserungen betreffen insbesondere Schlaf und Angst, Nebenwirkungen häufig die Kognition, Vigilanz und Psychomotorik.

5. Viele Patienten wünschen nach der Verordnung von Schlaf- oder Beruhigungsmitteln im Krankenhaus eine weiterführende Einnahme zu Hause. 


\section{Methodisches Vorgehen}

\subsection{Studiendesign}

Im Rahmen einer Querschnittsstudie („cross-sectional“) sollten Patienten $\geq 65$ Jahre mit Hilfe eines elektronischen Fragebogens (siehe Anlage 9.1) zur Einnahme von Schlaf- und Beruhigungsmitteln im Krankenhaus und zu Hause befragt werden. Die konsekutive Rekrutierung fand von Mai bis September 2014 statt. Von Montag bis Freitag (exklusive Feiertage) sollten Patienten jeweils ein bis zwei Tage vor oder am Tag ihrer Entlassung um Teilnahme am Interview gebeten werden. Die Befragung von Patienten, deren Entlassung am Wochenende oder an einem Feiertag geplant war, wurde auf den Freitag bzw. auf den Tag vor dem Feiertag vorgezogen. Patienten, die vor ihrer Entlassung aus organisatorischen Gründen nicht rechtzeitig erreicht werden konnten, wurden nicht nachträglich kontaktiert. Rekrutiert wurden Patienten aller Abteilungen des Evangelischen Krankenhauses Göttingen-Weende. Nach schriftlicher und mündlicher Präsentation des Projektes gaben die Chefärzte der beteiligten Fachbereiche ihr Einverständniserklärung zur Studie.

\subsection{Stellenwert der Untersuchung im Gesamtprojekt}

Die Studie ist Teil des Projekts „Schlaf- und Beruhigungsmittel an der Schnittstelle von Krankenhaus und Hausarzt“ des Instituts für Allgemeinmedizin der Universitätsmedizin Göttingen in Kooperation mit dem Evangelischen Krankenhaus Göttingen-Weende und dem Medizinischen Dienst der Krankenkassen Mecklenburg-Vorpommern e.V. Das Projekt wurde vom Bundesministerium für Gesundheit gefördert.

Eine zentrale Annahme des Projekts ist, dass manche ältere Patienten ein Schlaf- und Beruhigungsmittel zum ersten Mal in einer Akutsituation des Krankenhauses erhalten und die Erfahrung im Krankenhaus zu dem Wunsch führt, diese Medikamente nach Entlassung weiter einnehmen zu wollen. Ziel des Projekts ist zum einen eine quantitative Erfassung der verordneten Schlaf- und Beruhigungsmittel während des Aufenthalts im Krankenhaus bzw. bei Entlassung und zum anderen die Auswertung der Erfahrungen mit diesen Medikamenten aus Sicht der Patienten sowie aus Sicht von Krankenhausärzten, Hausärzten und Pflegepersonal. In Konsequenz der Ergebnisse sollen sich geeignete Interventionen im Evangelischen Krankenhaus Göttingen-Weende anschließen (Heinemann et al. 2016b).

Die vorliegende Arbeit stellt als Teilvorhaben des Gesamtprojektes die persönlichen Einschätzungen von älteren Patienten mit Schlaf- und Beruhigungsmitteln in den Mittelpunkt. Neben den Erfahrungen des medizinischen Personals und einer Auswertung der Akten zu der Verordnung von Schlaf- und Beruhigungsmittel soll demnach hier die subjektive Wahrnehmung der Patienten im Fokus stehen. Die Perspektive älterer Patienten und die Gründe 
für eine Einnahme bzw. eine Weitereinnahme sollen besser verstanden werden, um spätere Interventionen zielgenau planen und ausrichten zu können. Dafür war eine für das Krankenhaus möglichst repräsentative Zahl von Patienten für die Befragung zu gewinnen.

\subsection{Ort der Befragung}

Das Evangelische Krankenhaus Göttingen-Weende ist ein Krankenhaus der Grund- und Regelversorgung, das zugleich Lehrkrankenhaus der angrenzenden Universitätsmedizin Göttingen ist. Zum Zeitpunkt der Befragung hatte das Krankenhaus an zwei Standorten (in Göttingen-Weende und Bovenden-Lenglern) 601 Betten und beschäftigt rund 1100 Mitarbeiterinnen und Mitarbeiter, davon über 140 Ärzte bzw. Ärztinnen und ca. 440 Krankenschwestern und -pfleger. Mittlerweile ist ein weiterer Standort (Krankenhaus Neu-Mariahilf in Göttingen-Stadt) hinzugekommen. Laut der internen Statistik des Evangelischen Krankenhaus Göttingen-Weende hatte dieses im Jahr 2014 insgesamt 20877 Patientenfälle, davon waren mehr als die Hälfte Patienten im Alter ab 65 Jahren. Folgende Abteilungen wurden in die Studie einbezogen: Innere Medizin, Geriatrie, Unfallchirurgie/Orthopädie, Allgemein/Viszeral-/Thorax- und Minimal-invasive Chirurgie, Plastische Chirurgie, Urologie und HalsNasen- Ohrenheilkunde. Die Verteilung der Fachbereiche auf die 12 Stationen war wie folgt: drei Stationen der Inneren Medizin, zwei Stationen und die Hälfte einer weiteren der Unfallchirurgie, je eine Station der Urologie und Allgemeinchirurgie, eine gemeinsame Station der Hals-Nasen-Ohren-Heilkunde und plastischen Chirurgie sowie drei Stationen und die Hälfte einer weiteren der Geriatrie. Ausgeschlossen von der Befragung waren die Intensiv- und die Intermediate-Care-Stationen. Als Krankenhaus, das keine Universitätsklinik ist, bietet das Evangelische Krankenhaus Göttingen-Weende das Patientenspektrum eines durchschnittlich mittelgroßen deutschen Krankenhauses mit typischen Fachbereichen. Das geriatrische Zentrum des Evangelischen Krankenhauses Göttingen-Weende betreut mit 96 Betten eine große Anzahl von Patienten - u. a. deshalb, weil es eine vergleichbare Abteilung in der angrenzenden Universitätsklinik bisher nicht gibt.

\subsection{Patientenbefragung}

Das Einnahmeverhalten und die Erfahrungen von älteren Patienten mit Schlaf- und Beruhigungsmitteln während des Aufenthalts in einem Krankenhaus und zu Hause sollten in dieser Studie per Fragebogen quantitativ erfasst werden. Da die Teilnahmequote bei einem selbstständigen, unbegleiteten Ausfüllen eines Fragebogens erfahrungsgemäß relativ niedrig ist und es keine Möglichkeit für den Studienteilnehmer gibt, Nachfragen zum Verständnis der Fragen zu stellen, erschien die mündlich-persönliche Befragung mit Hilfe eines elektroni- 
schen, standardisierten Fragebogens eine erfolgsversprechende Variante für die Erhebung zu sein.

\subsubsection{Standardisierte Befragung}

Zur Optimierung des Studienergebnisses wurde als Erhebungsmethodik die persönliche standardisierte Befragung gewählt mit einer festgelegten Anzahl von Fragen, feststehenden Formulierungen für die Ansprache und einer klaren Anordnung der Fragen. Im Begleitschreiben wurden Ziel, Verantwortlichkeiten und Vorgehensweise bei der Befragung erläutert. Die Face-to-Face-Befragung wurde mit Hilfe eines Fragebogens - eines sogenannten CAPI (computer assisted personal interview) - durchgeführt. Dabei wurden die einzelnen Fragen des elektronischen Fragebogens den an der Studie Teilnehmenden im Sinne einer konsequenten Standardisierung wortwörtlich vorgelesen. Die Befragung wurde von der Verfasserin sowie einer weiteren wissenschaftlichen Mitarbeiterin des Instituts für Allgemeinmedizin der Universitätsmedizin Göttingen umgesetzt. Die Mitarbeiterin hatte Erfahrung mit PatientenInterviews und war für die spezifischen Anforderungen der Befragung in dieser Studie geschult worden.

\section{Verhaltensregeln für das Interview}

Die standardisierte Befragung eröffnete die Interviewerin mit einer Begrüßung, persönlichen Vorstellung, kurzen Erläuterung der Studie sowie einer Aufklärung über die rechtlichen Rahmenbedingungen. Beide Interviewerinnen leiteten die Befragung mit derselben Erklärung zur Studie ein und trugen einen weißen Kittel.

Vor Beginn der Befragung wurden die Patienten über den Zweck der Befragung informiert und erhielten ein Informationsblatt, die Erläuterungen zur Verknüpfung ihrer Angaben mit den Verordnungsdaten des Krankenhauses (Patientenakten) sowie die Einverständniserklärung zur Teilnahme. Die Patienten wurden zur Befragung direkt am Krankenbett aufgesucht oder in einen geeigneten Raum auf der Station befragt.

\subsubsection{Entwicklung des Fragebogens}

Der für die Befragung verwendete Fragebogen (siehe Anlage 9.1) wurde auf Grundlage der Forschungshypothesen, international vergleichbarer Studien und insbesondere in Anlehnung an die Studie von Siriwardena et al. (2008) entwickelt. Zu Beginn der Dissertation war die Entwicklung des Fragebogens bereits sehr fortgeschritten. Die Dissertation umfasst aber noch dessen Finalisierung, insbesondere die Anpassung an die Rahmenbedingungen des Krankenhauses, an die aktuellen Erkenntnisse der Forschung und die konkrete Umsetzung als CAPI. In Zusammenarbeit mit dem Geschäftsbereich Informationstechnologie der Universitätsmedizin Göttingen wurde dafür der zunächst papiergebundene Fragebogen in ein computergestütztes Fragenprogramm mit dem Programm Microsoft Access 2007 überführt. 
Hauptverantwortlicher hierfür war in der Abteilung Dipl.-Informatiker Fred Viezens, unterstützt von Luca Hernandez Acosta.

\subsubsection{Pilotstudie}

In einer Pilotstudie wurden zunächst an einem Tag 15 Patienten im Alter von 65 Jahren und älter auf der geriatrischen Station 5.2 im Evangelischen Krankenhaus Göttingen-Weende befragt. Verständnis- und Nachfragen von Patienten führten zu kleinen Änderungen und entsprechenden technischen Anpassungen des Fragebogens. Da in der Pilotstudie außerdem mehrere Patienten eine Verbesserung der Toleranz der den Schlaf störenden Umgebungsfaktoren im Krankenhauses durch die Einnahme von Schlaf- oder Beruhigungsmitteln äußerten, wurde dieser Aspekt in dem Fragebogen unter der Frage 4 berücksichtigt (siehe Anlage 9.1).

\subsubsection{Der Fragebogen}

Der elektronische standardisierte Fragebogen (siehe Anlage 9.1) bestand aus vier Hauptabschnitten.

Der erste Abschnitt beinhaltet die persönliche Befragung zur Einnahme und subjektiven Erfahrung mit Beruhigungs- und Schlafmitteln während des aktuellen Krankenhausaufenthalts:

- Verordnung, Name und Anzahl der Präparate sowie Dauer der Einnahme von Schlafund Beruhigungsmitteln,

- $\quad$ wahrgenommene Verbesserungen und Nebenwirkungen durch die Einnahme,

- Wunsch der Weitereinnahme nach Entlassung,

- Beurteilung der Schlaf- und Beruhigungsmittel insgesamt.

Der zweite Abschnitt sollte die Erfahrungen der Patienten mit Schlaf- und Beruhigungsmitteln zu Hause bzw. vor der stationären Aufnahme erfassen:

- Einnahme zu Hause, Name und Anzahl der Präparate, Einnahme in den letzten sechs Monaten und Einnahmezeitraum von Schlaf- und Beruhigungsmitteln,

- wahrgenommene Verbesserungen und Nebenwirkungen durch die Einnahme,

- Wunsch der Weitereinnahme nach Entlassung,

- Versuch des Absetzens,

- Beurteilung der Schlaf- und Beruhigungsmittel insgesamt.

Im dritten Abschnitt sollten die demographischen Daten der Patienten erhoben werden. Ermittelt wurden das Alter der Patienten, das Geschlecht und die behandelnde Fachabteilung. 
In einem vierten Abschnitt wurden tabellarisch die Einnahme der Medikamente und deren Häufigkeit dokumentiert. Nicht miteinbezogen werden sollten Hypnotika, die zur Prämedikation vor einem chirurgischen oder diagnostischen Eingriff verordnet wurden. Die Häufigkeiten wurden mit „nie“, „einmalig“, „mehrmals mit Unterbrechung“ oder „täglich“ skaliert.

Folgende Medikamente wurden erfasst:

- langwirksame Benzodiazepine: Diazepam, Flurazepam, Bromazepam, Nitrazepam, Flunitrazepam,

- $\quad$ kurz- und mittellangwirksame Benzodiazepine: Temazepam, Lorazepam, Oxazepam, Lormetazepam,

- Z-Substanzen: Zolpidem, Zopiclon,

- Baldrianpräparate, Mirtazapin und alle weiteren alternativen Schlafmittel.

\subsubsection{Gedankenprotokolle}

Zusätzlich zu der computergestützten Befragung notierten sich die Interviewerinnen Aussagen von Patienten zu ihrer Schlafsituation als Gedankenprotokolle. Diese umfassten u. a. persönliche Einschätzungen von Schlaf- oder Beruhigungsmitteln, Gründe für die Einnahme und Berichte über Absetzversuche.

\subsubsection{Ein- und Ausschlusskriterien}

Folgende Kriterien mussten für die Befragung erfüllt werden: Die Patienten sollten kurz vor ihrer Entlassung aus dem Krankenhaus stehen, klar und orientiert sowie 65 Jahre oder älter sein und einen Aufenthalt im Krankenhaus von mindestens 3 Nächten gehabt haben.

Von der Befragung ausgeschlossen wurden Patienten mit mangelnden Sprachkenntnissen oder stark eingeschränkten kognitiven Fähigkeiten (z.B. durch eine Demenzerkrankung). Die Häufigkeit dieser Ausschlüsse sollte - so gut wie möglich - dokumentiert werden.

Alle Patienten sollten auf den Stationen vom pflegerischen und ärztlichen Personal auf Einund Ausschlusskriterien hin geprüft und anschließend von den Interviewerinnen um Teilnahme gebeten werden.

\subsection{Datenauswertung}

Die Auswertung der Daten erfolgte mit dem Statistikprogramm Statistical Analysis System (SAS) 9.4 .

\subsubsection{Datenaufbereitung}

Für die Auswertung wurden die Fachbereiche des Evangelischen Krankenhauses GöttingenWeende in die Kategorien „Innere Medizin“, „Geriatrie“ und „Chirurgische Fächer" unterteilt. 
Diese Einteilung sollte Vergleiche unter diesen drei Kategorien gestatten. Unter „Chirurgische Fächer" wurden zusammengefasst: Unfallchirurgie, Urologie, Allgemeinchirurgie, Plastische Chirurgie und Hals-, Nasen-, Ohrenheilkunde.

Die Einteilung der Altersgruppen erfolgte in zwei Kategorien gemäß Vorschlag des Robert Koch-Instituts: Patienten zwischen 65 und 84 Jahren sind danach „junge Alte“ und Patienten im Alter von 85 Jahren und älter „alte Alte“ (Böhm et al. 2009).

Zur Vorbereitung der statistischen Analyse wurden die Daten durch sogenannte arrayFunktionen in SAS sortiert. Dies bedeutet, dass Daten in verschiedene Untergruppen zusammengefasst wurden, um sie geclustert auswerten zu können. So wurde zum Beispiel der Anteil der Patienten, die erfahrene Nebenwirkungen und gleichzeitig den Wunsch nach einer weiteren Einnahme zu Hause angegeben hatten, bestimmt.

\subsubsection{Statistische Analyse}

Die statistische Auswertung mit SAS 9.4 nutzte vier analytische Prozeduren:

1. Häufigkeitsauszählungen inklusive Gruppenvergleiche mit Erstellung klassischer Kreuztabellen (proc freq bei SAS)

2. Bestimmung des arithmetischen Mittels und der Standardabweichung (proc means bei SAS)

3. Logistische Regression zur Untersuchung von möglichen Einflussfaktoren auf den Wunsch nach Weitereinnahme der Schlaf- und Beruhigungsmittel nach dem Krankenhausaufenthalt. Für die Analyse des Risikoverhältnisses wurden sowohl univariate als auch multivariate Odds Ratios bestimmt, um einen Bias durch eventuelle Störvariablen zu vermeiden (proc logistic bei SAS).

4. Berechnung des Cohen's Kappa, um die Übereinstimmung von Patientenaussagen mit den Angaben in der Patientenakte zu prüfen (agree-Option von proc freq bei SAS): Kappa schätzt die Interrater-Reliabilität - also den Anteil der Übereinstimmungen -, die um die zufällig zu erwartenden Übereinstimmungen korrigiert werden (Fleiss und Cohen 1973). Der Wert des Cohen's Kappa, der zwischen 0 und 1 liegen kann, wird anhand der Einteilung von Landis und Koch (1977) bewertet. Je näher Kappa an der 1 liegt, umso verlässlicher:

- unter 0: schwache Übereinstimmung (,poor“)

- 0,00-0,20: leichte Übereinstimmung („slight“)

- 0,21-0,40: ausreichende Übereinstimmung (,fair“)

- 0,41-0,60: mittelmäßige Übereinstimmung („moderate“)

- 0,61-0,80: beachtliche Übereinstimmung („substantial“)

- 0,81 - 1,00: fast perfekte Übereinstimmung (,almost perfect“). 


\subsection{Datenschutz und Genehmigung durch die Ethik-Kommission}

Beide Interviewerinnen haben im Evangelischen Krankenhaus Göttingen-Weende eine Datenschutzerklärung abgelegt zur strikten Verschwiegenheit in Bezug auf persönliche und medizinische Daten von Patienten (siehe Anlage 9.2). Die Zulassung der Studie durch die Ethik-Kommission der Medizinischen Fakultät der Georg-August-Universität Göttingen wurde im Februar 2014 beantragt und im Mai 2014 unter der Aktennummer 25/2/14 genehmigt. 


\section{Ergebnisse}

\subsection{Teilnahme}

Gemäß Vereinbarung mit dem Evangelischen Krankenhaus Göttingen-Weende sollte das medizinische Personal (Ärzte oder Pflegende) auf den beteiligten Stationen den Interviewerinnen die potenziell zu befragenden Patienten benennen - gemäß vereinbarten Ein- bzw. Ausschlusskriterien (vgl. Kapitel 4.4.6). Für eine korrekte Erfassung der Grundgesamtheit (,population at risk“) wäre optimal gewesen, wenn im Befragungszeitraum alle Patienten über 65 Jahre kurz vor deren Entlassung sowie alle durch das medizinische Personal gemäß Ausschlusskriterien ausgenommenen Patienten auf einer Liste vermerkt worden wären. Um den Aufwand für das Personal auf den beteiligten Stationen jedoch möglichst gering zu halten und auch die Krankenhausroutine nicht empfindlich zu stören, wurde auf dieses arbeitsaufwendige Vorgehen verzichtet. Es kann dennoch davon ausgegangen werden, dass die Rekrutierung tatsächlich entsprechend der getroffenen Vereinbarung erfolgte, was einzelne Verletzungen des Protokolls nicht ausschließt. Definitiv bekannt ist, dass 696 Patienten die Ein- und Ausschlusskriterien laut Angaben des medizinischen Personals erfüllten und von den Interviewerinnen um Teilnahme an der Studie gebeten wurden; 508 Patienten (73,0\%) erklärten sich zu einer Befragung bereit, 188 Patienten lehnten diese ab.

Aufgrund der regelmäßigen Anwesenheit mindestens einer der Interviewerinnen im Erhebungszeitraum konnten zumindest bei einem Teil der Patienten die Gründe, die das Personal zum Ausschluss von der Befragung bewogen hatte, nachvollzogen bzw. dokumentiert werden. Dies gelang bei 261 Patienten; geschätzt dürfte es sich dabei um ca. ein Drittel bis die Hälfte aller ausgeschlossenen Patienten handeln. Die häufigsten Ausschlussgründe waren räumliche und zeitliche Desorientierung sowie bekannte Demenzerkrankungen (Tabelle 1).

\subsection{Stichprobe}

Von den 508 Studienteilnehmern waren 300 Frauen (59,1\%). Nach der Einteilung in „Innere Medizin“, „Geriatrie“ und „operative Fächer" war die Verteilung der Studienteilnehmer recht ausgewogen - mit knapp der Hälfte der Teilnehmer aus den operativen Fachbereichen und fast je einem Viertel aus der Geriatrie und Inneren Medizin (Tabelle 2). Das Durchschnittsalter der befragten Patienten betrug 77,1 Jahre (Standardabweichung: 7,2; Range: 65 bis 97). Die befragten Patienten der Geriatrie waren mit 81,4 Jahren im Mittel erwartungsgemäß am ältesten (Tabelle 2). 
Tabelle 1: Ausschluss von der Befragung

\begin{tabular}{lcc}
\hline & \multicolumn{2}{c}{ Patienten } \\
\cline { 2 - 3 } Ausschlussgrund & $\mathbf{n}$ & $\%$ \\
\hline Räumliche und zeitliche Desorientierung & 197 & 75,5 \\
Demenzerkrankung & 33 & 12,6 \\
Sprachbarriere & 15 & 5,7 \\
Sehbehinderung & 12 & 4,6 \\
Als „präfinal“ eingeschätzt & 2 & 0,8 \\
Schreibbehinderung & 2 & 0,8 \\
\hline Alle & $\mathbf{2 6 1}$ & $\mathbf{1 0 0}$ \\
\hline
\end{tabular}

Tabelle 2: Stichprobe

\begin{tabular}{lccccc}
\hline & \multicolumn{2}{c}{ Patienten } & & \multicolumn{2}{c}{ Alter } \\
\cline { 2 - 3 } \cline { 5 - 6 } \cline { 5 - 6 } Frauen & $\mathbf{n}$ & $\%$ & & Mittelwert & SD*$^{\star}$ \\
Männer & 300 & 59,1 & & 77,8 & 7,2 \\
Chirurgische Fächer & 252 & 49,7 & & 75,1 & 6,3 \\
Innere Medizin & 140 & 27,6 & & 77,1 & 7,3 \\
Geriatrie & 115 & 22,7 & & 81,4 & 7,0 \\
\hline Alle & $\mathbf{5 0 8}$ & $\mathbf{1 0 0}$ & & $\mathbf{7 7 , 1}$ & $\mathbf{7 , 2}$ \\
\hline
\end{tabular}

* Standardabweichung;

**eine fehlende Angabe zur Abteilungszugehörigkeit; deshalb ergibt die Summe der drei Fachbereiche nur $\mathrm{N}=507$.

\subsection{Einnahme von Schlaf- oder Beruhigungsmitteln während des Krankenhausauf- enthalts}

Fast die Hälfte der befragten Patienten (227/508) hatte - laut eigener Aussage - mindestens einmal Schlaf- oder Beruhigungsmittel während ihres Krankenhausaufenthalts erhalten. Unter den Geschlechtern ließ sich kein Unterschied feststellen. Deutlich mehr Patienten der „Geriatrie“ berichteten von einer Einnahme; Patienten der zusammengefassten Fachabteilungen „Chirurgische Fächer“ oder „Innere Medizin“ erhielten seltener ein entsprechendes 
Medikament (Tabelle 3). Ein Unterschied zwischen den beiden Altersgruppen ließ sich nicht feststellen.

Ein Viertel aller Patienten (58/227), die ein Schlaf- oder Beruhigungsmittel laut eigener Aussage erhalten hatten, konnte den Namen des Medikaments nennen. Am häufigsten genannt wurden Medikamente mit den Wirkstoffen Baldrian, Mirtazapin und Lormetazepam. Zur Einnahmefrequenz gab immerhin mehr als die Hälfte der Patienten (116/227) eine tägliche Einnahme an, ein Drittel (80/227) hatte Schlaf- oder Beruhigungsmittel mehrmals mit Unterbrechungen erhalten. Die überwiegende Mehrheit (86,3\%) der 227 Patienten hatte nicht mehr als ein Medikament erhalten. Zwischen den Geschlechtern gab es bei dieser Frage keine Unterschiede.

Tabelle 3: Einnahme von Schlaf- oder Beruhigungsmitteln im Krankenhaus

\begin{tabular}{lcc}
\hline & $\begin{array}{c}\text { Patienten mit mind. einem Schlaf- } \\
\text { oder Beruhigungsmittel }\end{array}$ \\
\cline { 2 - 3 } & $\mathbf{n}$ & $\%$ \\
\hline Frauen (N=293) & 136 & 45,3 \\
Männer (N=200) & 91 & 43,8 \\
Chirurgische Fächer (N=245) & 99 & 39,3 \\
Innere Medizin (N=137) & 66 & 47,1 \\
Geriatrie (N=110) & 61 & 53,0 \\
65-84 Jahre (N=420) & 193 & 45,0 \\
$\geq 85$ Jahre (N=73) & 34 & 43,0 \\
\hline Alle (N=508*) & $\mathbf{2 2 7}$ & $\mathbf{4 4 , 7}$ \\
\hline
\end{tabular}

Genannt werden jeweils diejenigen Patienten, die laut eigener Aussage mindestens ein Schlaf- oder Beruhigungsmittel erhielten;

*15 Patienten hatten mit „weiß nicht“ geantwortet.

\subsection{Wahrgenommene Verbesserungen und Nebenwirkungen durch die Einnahme von Schlaf- und Beruhigungsmitteln während des stationären Aufenthalts}

Durch die Einnahme des Schlaf- oder Beruhigungsmittels hatten fast $90 \%$ der Patienten (201/226) mindestens eine Verbesserung wahrgenommen, am häufigsten Studienteilnehmer der geriatrischen Stationen (95,1\%). Bei einer Mehrzahl der Patienten verbesserten sich 
Einschlafstörungen $(73,1 \%)$ und Durchschlafstörungen $(59,0 \%)$. Eine Verbesserung von Angstzuständen spielte eine untergeordnete Rolle (Abbildung 1). Betrachtet man die Verbesserungen von Einschlaf- und/oder Durchschlafstörungen gemeinsam, so konnte die groBe Mehrheit der Patienten (81,1\%) eine positive Beeinflussung dieser Symptome auf die Einnahme von Schlaf- oder Beruhigungsmittel zurückführen.

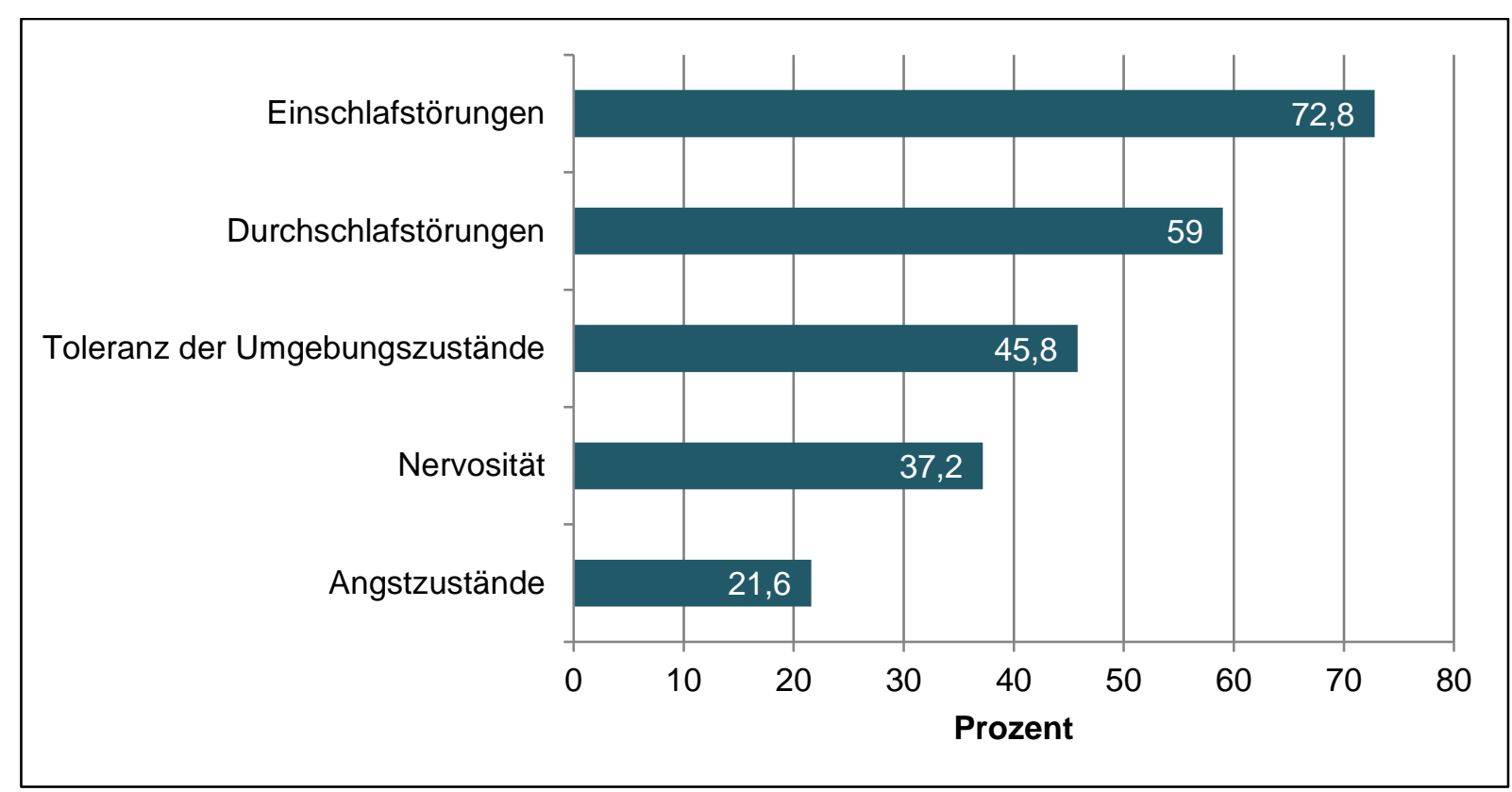

Abbildung 1: Wahrgenommene Verbesserungen durch die Einnahme eines Schlaf- oder Beruhigungsmittels ( $N=226$; Patienten konnten mehr als eine Antwort geben)

Von Nebenwirkungen durch die Einnahme berichteten knapp $40 \%(87 / 226)$ der Patienten, etwas häufiger in der Geriatrie (45,9\%) und der Inneren Medizin (42,4 \%). Ein geschlechtsspezifischer Unterschied ließ sich nicht erkennen (Tabelle 4). Schläfrigkeit wurde am häufigsten (20,3\%) als unerwünschte Arzneimittelwirkung genannt, gefolgt von Benommenheit (11\%) und Schwindel (9,7\%) (Abbildung 2).

Die Mehrheit (85,7 \%) der Patienten schätzte die Wirkung der eingenommenen Medikamente als insgesamt „eher positiv“ ein. Über ein Drittel der Patienten (85/227) wünschte sich, die erhaltenen Schlaf- oder Beruhigungsmittel nach Entlassung weiter einzunehmen. Immerhin $15,4 \%$ Patienten äußerten diesen Wunsch, obwohl sie mindestens eine Nebenwirkung durch die Einnahme angegeben hatten. 
Tabelle 4: Wahrnehmung mindestens einer Nebenwirkung

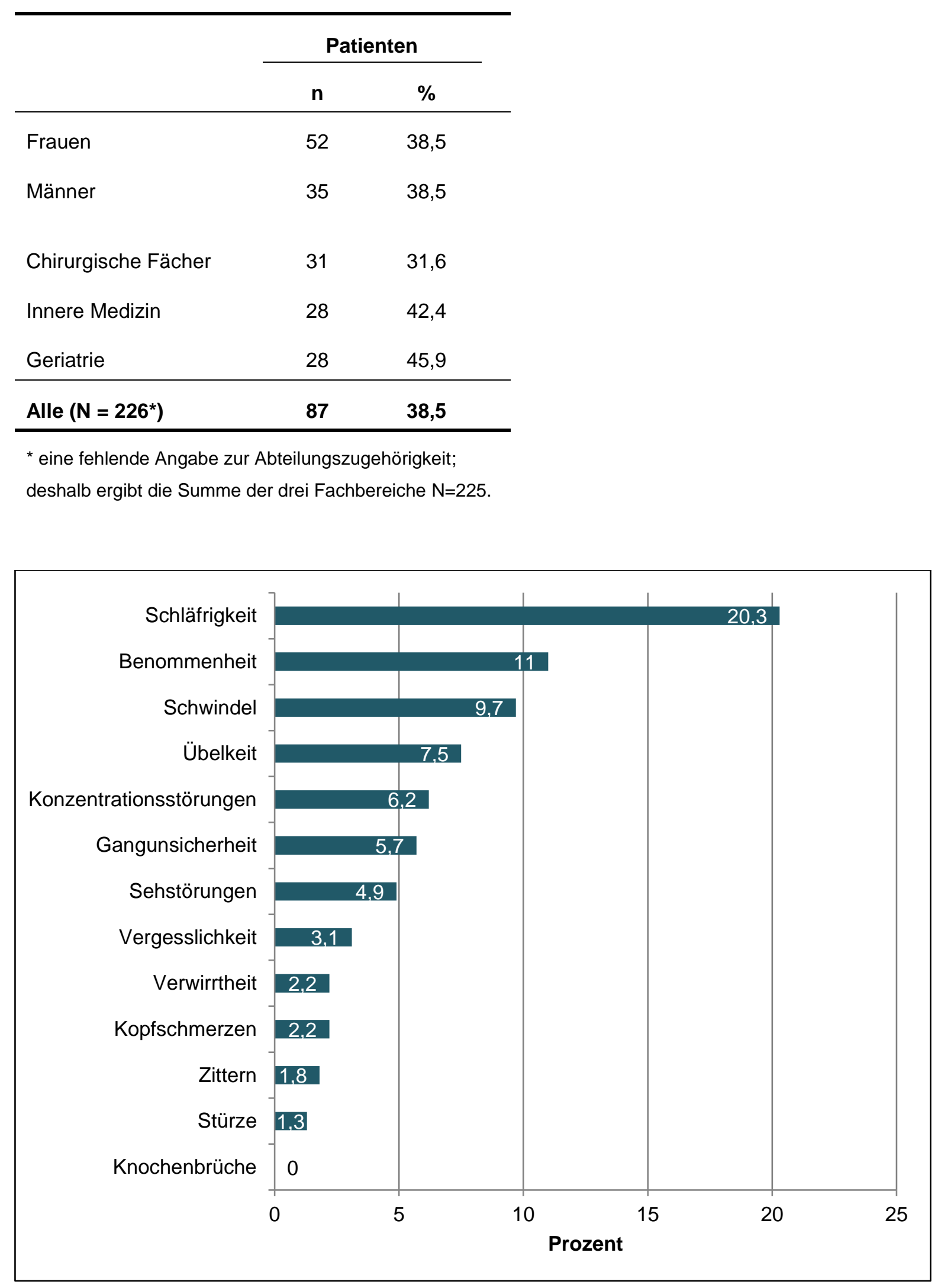

Abbildung 2: Nebenwirkungen von Schlaf- oder Beruhigungsmitteln im Krankenhaus ( $N=226$; Mehrfachantworten möglich.) 


\subsection{Einnahme von Schlaf- oder Beruhigungsmitteln vor dem Krankenhausaufent- halt}

Knapp 20 \% (89/508) der Patienten hatten bereits zu Hause Erfahrungen mit Schlaf- oder

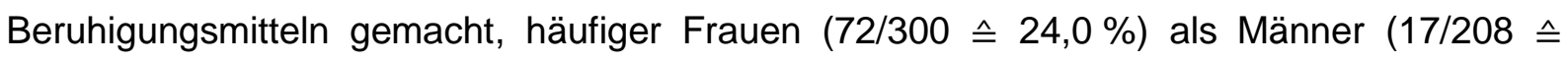
$8,2 \%)$. Viele von innen $(80 / 89 \hat{=} 89,9 \%)$ hatten die Medikamente auch in den letzten sechs Monaten eingenommen. Über zwei Drittel (61/89) hatten die Medikamente zu Hause länger als ein Jahr eingenommen.

Die große Mehrheit der Patienten mit Vorerfahrungen (73/89 $=82,0 \%$ ) erhielt auch im Krankenhaus Schlaf- oder Beruhigungsmittel. Für knapp ein Drittel (148/508) hingegen war es im Krankenhaus die erste Verordnung eines Schlaf- oder Beruhigungsmittels. Umgekehrt hatten $32,2 \%(73 / 227)$ derjenigen Patienten, die stationär Schlaf- oder Beruhigungsmittel nach eigener Aussage erhalten hatten, bereits häusliche Vorerfahrungen (Tabelle 5).

Tabelle 5: Verordnung während des Krankenhausaufenthalts und zu Hause

\begin{tabular}{|c|c|c|c|c|c|c|}
\hline \multirow[b]{3}{*}{ Verordnung im Krankenhaus } & \multicolumn{6}{|c|}{ Verordnung zu Hause } \\
\hline & \multicolumn{2}{|c|}{ ja } & \multicolumn{2}{|c|}{ nein } & \multicolumn{2}{|c|}{ weiß nicht } \\
\hline & $\mathbf{n}$ & $\%$ & $\mathbf{n}$ & $\%$ & $\mathbf{n}$ & $\%$ \\
\hline ja & 73 & 82,0 & 148 & 35,9 & 6 & 85,7 \\
\hline nein & 13 & 14,6 & 253 & 61,4 & 0 & 0,0 \\
\hline weiß nicht & 3 & 3,4 & 11 & 2,7 & 1 & 14,3 \\
\hline Alle $(\mathrm{N}=508)$ & 89 & 100 & 412 & 100 & 7 & 100 \\
\hline
\end{tabular}

Fast alle der Patienten (85/89) hatten zu Hause Verbesserungen durch die Einnahme von Schlaf- oder Beruhigungsmitteln wahrgenommen; etwa $40 \%$ (35/86) führten Nebenwirkungen auf deren Einnahme zurück. Dies entspricht prozentual den Antworten über die Nebenwirkungen durch die Schlaf- oder Beruhigungsmittel im Krankenhaus. Die Patienten wurden anschließend befragt, ob sie schon einmal versucht hätten, die Schlaf- oder Beruhigungsmittel abzusetzen. Knapp die Hälfte (43/89) der Befragten hatte die Einnahme schon einmal unterbrochen.

\subsection{Erfahrungsberichte der Patienten laut Gedankenprotokoll}

Neben den standardisierten Antworten schilderten einige Patienten ihre persönliche Einschätzung zu Schlaf- und Beruhigungsmitteln. Gemäß ergänzenden Gedankenprotokollen der beiden Interviewerinnen berichteten Patienten zum Beispiel über Angst vor den Neben- 
wirkungen und einer möglichen Sucht nach diesen Medikamenten. Mehrere Patienten hatten Erfahrungen mit jahrelanger Einnahme und Absetzversuchen, Entzugssymptomen. Gleichzeitig rechtfertigten sie dezidiert die Einnahme der Medikamente, insbesondere auch für zu Hause. Sie beschrieben kreisende Gedanken, Zukunftsängste, Traumatisierungen in der Vergangenheit und Schwierigkeiten, im Bett zur Ruhe zu kommen. Um überhaupt „abschalten“ zu können, sei die Schlaftablette notwendig.

\subsection{Dokumentation der Schlaf- oder Beruhigungsmittel in der Patientenakte}

Laut Patientenakten hatte knapp die Hälfte (235/508) der befragten Patienten mindestens ein Schlaf- oder Beruhigungsmittel erhalten, deutlich häufiger in der Geriatrie (Tabelle 6). Die Patienten, die 85 Jahre und älter waren, hatten etwas häufiger ein Schlaf- oder Beruhigungsmittel erhalten als die „jungen alten“ Patienten. Ein Unterschied zwischen den Geschlechtern ließ sich nicht feststellen (Tabelle 6).

Tabelle 6: Medikamenteneinnahme laut Dokumentation in der Patientenakte

\begin{tabular}{|c|c|c|}
\hline & \multicolumn{2}{|c|}{ Anzahl } \\
\hline & $\mathbf{n}$ & $\%$ \\
\hline Frauen $(\mathrm{N}=300)$ & 142 & 47,3 \\
\hline Männer (N=208) & 93 & 44,7 \\
\hline Chirurgische Fächer $(\mathrm{N}=252)$ & 94 & 37,3 \\
\hline Innere Medizin ( $N=140)$ & 67 & 47,9 \\
\hline Geriatrie $(\mathrm{N}=115)$ & 73 & 63,5 \\
\hline 65-84 Jahre (N=429) & 192 & 44,8 \\
\hline$\geq 85$ Jahre $(\mathrm{N}=79)$ & 43 & 54,4 \\
\hline Alle $\left(N=508^{\star}\right)$ & $235^{*}$ & 46,3 \\
\hline
\end{tabular}

Nach Einteilung in die Stoffgruppen wurden laut Akte am häufigsten Benzodiazepine

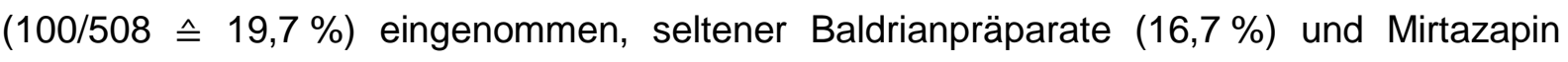
$(12,2 \%)$ und Z-Substanzen (11,2\%). Von allen befragten Patienten hatte knapp ein Drittel (150/508) mindestens einmal ein Benzodiazepinderivat oder eine Z-Substanz eingenommen (Tabelle 7). Sieben von innen hatten stationär sowohl Benzodiazepine als auch Z- 
Substanzen erhalten. Laut Akte hatten geriatrische Patienten (34,8\%) Arzneimittel dieser Stoffgruppen häufiger eingenommen als Patienten der Chirurgischen Fächer oder der Inneren Medizin. Zwischen Männern und Frauen konnte kein Unterschied festgestellt werden. Ebenso zeigte sich kein Alterseffekt. $\mathrm{Zu}$ den am häufigsten eingenommenen alternativen Schlaf- oder Beruhigungsmitteln gehörten Baldrianpräparate und das sedierende Antidepressivum Mirtazapin. Baldrianpräparate wurde besonders häufig in der Inneren Medizin $(30,7 \%)$ und Geriatrie $(22,6 \%)$ verordnet, in den Chirurgischen Fächern hingegen nur selten. Patienten über 85 Jahre erhielten Baldrianarzneimittel etwas häufiger als die ,jungen alten“ Patienten. Unterschiede zwischen den Geschlechtern zeigten sich bei der Einnahme von Baldrianpräparaten nicht. Mirtazapin wurde dagegen etwas häufiger von Frauen eingenommen und häufiger auf den Stationen der Geriatrie verordnet. Insbesondere die sehr alten Patienten erhielten dieses Medikament (Tabelle 7).

Tabelle 7: Einnahme von Schlaf- und Beruhigungsmitteln laut Dokumentation

\begin{tabular}{|c|c|c|c|c|c|c|c|c|c|c|c|c|}
\hline & \multicolumn{2}{|c|}{ Benzo* } & \multicolumn{2}{|c|}{ Z-Sub.** } & \multicolumn{2}{|c|}{ BenzoZ $^{\star \star \star}$} & \multicolumn{2}{|c|}{ Baldrian } & \multicolumn{2}{|c|}{ Mirtazapin } & \multicolumn{2}{|c|}{ Alle } \\
\hline & $\mathbf{n}$ & $\%$ & $\mathbf{n}$ & $\%$ & $\mathbf{n}$ & $\%$ & $\mathbf{n}$ & $\%$ & $\mathbf{n}$ & $\%$ & $\mathbf{n}$ & $\%$ \\
\hline Frauen & 59 & 19,7 & 35 & 11,7 & 90 & 30,0 & 51 & 17,0 & 42 & 14,0 & 142 & 47,3 \\
\hline Männer & 41 & 19,7 & 22 & 10,6 & 60 & 28,9 & 34 & 16,4 & 20 & 9,6 & 93 & 44,7 \\
\hline Chirurgische Fächer & 71 & 28,1 & 8 & 3,2 & 79 & 31,4 & 15 & 6,0 & 8 & 3,2 & 94 & 37,3 \\
\hline Innere Medizin & 16 & 11,4 & 17 & 12,1 & 31 & 22,1 & 43 & 30,7 & 10 & 7,1 & 67 & 47,9 \\
\hline Geriatrie & 13 & 11,3 & 32 & 27,8 & 40 & 34,8 & 26 & 22,6 & 43 & 37,4 & 73 & 63,5 \\
\hline Junge alte Patienten & 90 & 21,0 & 42 & 9,8 & 128 & 29,8 & 69 & 16,1 & 41 & 9,6 & 192 & 44,8 \\
\hline Alte alte Patienten & 10 & 12,7 & 15 & 19,0 & 22 & 27,9 & 16 & 20,3 & 21 & 26,6 & 43 & 54,4 \\
\hline Alle $\left(N=508^{\star \star \star \star}\right)$ & 100 & 19,7 & 57 & 11,2 & 150 & 29,5 & 85 & 16,7 & 62 & 12,2 & 235 & 46,2 \\
\hline
\end{tabular}

*Benzodiazepine, ${ }^{* \star}$ Z-Substanzen; ${ }^{* * *}$ Benzodiazepine und Z-Substanzen; ${ }^{* * *}$ eine fehlende Angabe zur Abteilungszugehörigkeit; deshalb ergibt die Summe der drei Fachbereiche $\mathrm{N}=507$.

Nach einzelnen Präparaten aufgegliedert wurden Baldrianarzneimittel am häufigsten eingenommen; danach folgen das Benzodiazepin Lormetazepam und das Antidepressivum Mirtazapin an dritter Stelle. Lormetazepam war das am häufigsten erfasste Benzodiazepinderivat. 82 Patienten (16,1\%) hatten dieses Medikament erhalten. Unter den Z-Substanzen wurde laut Akte Zolpidem am häufigsten eingenommen (Abbildung 3). 


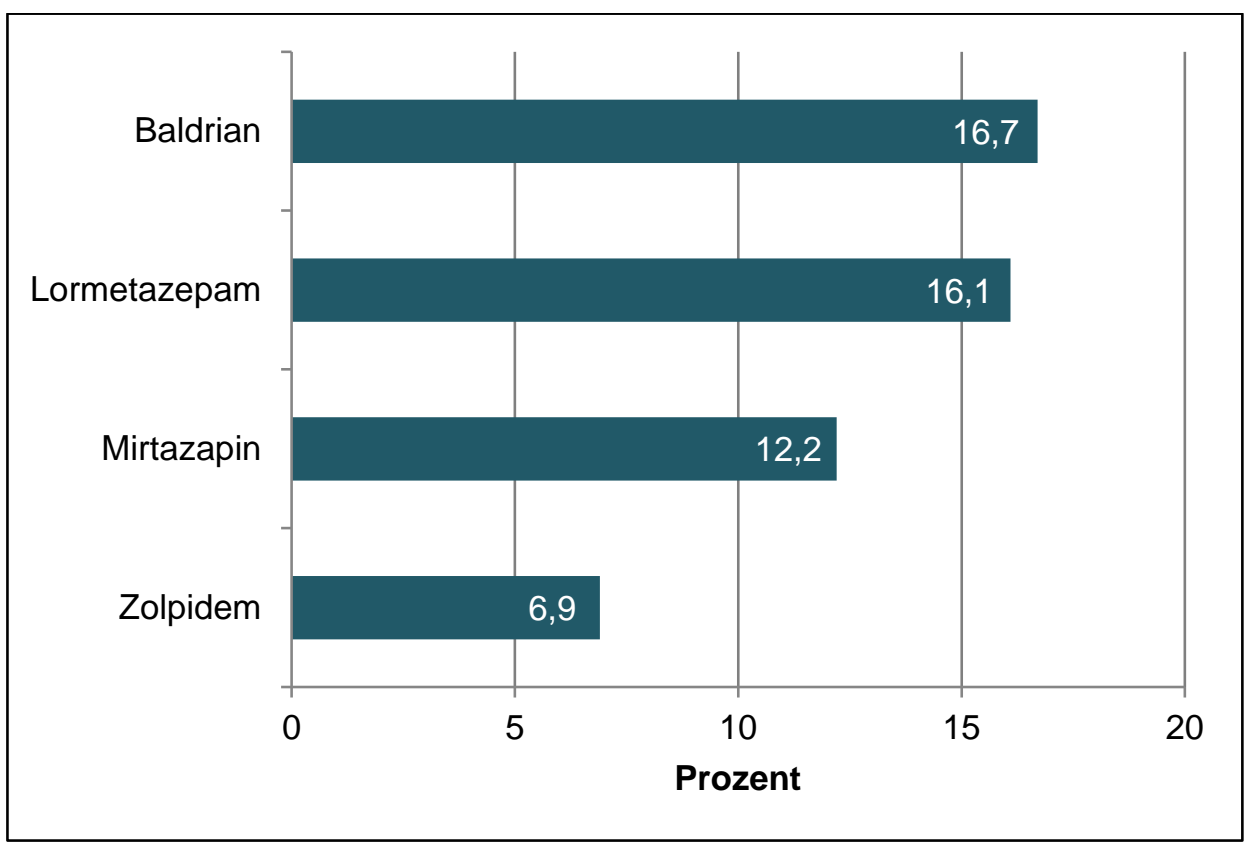

Abbildung 3: Am häufigsten verordnete Medikamente laut Patientenakte $(N=508)$

\subsection{Vergleich der Patientenakte mit der Patientenaussage}

Vergleicht man die Dokumentation der Einnahme mit den subjektiven Aussagen in der Patientenbefragung, zeigt sich eine große Übereinstimmung: Der Großteil (84,6\%) der Patientenaussagen aus der Befragung stimmt sehr gut mit der Dokumentation (Cohen's Kappa: 0,70 ) überein (Tabelle 8).

Tabelle 8: Vergleich der Patientenaussage über die Einnahme mit der Dokumentation

\begin{tabular}{lcccccc}
\hline & \multicolumn{3}{c}{ Einnahme laut Dokumentation } \\
\cline { 2 - 4 } \cline { 5 - 7 } Einnahme laut Patientenaussage & & \multicolumn{2}{c}{ ja } & & \multicolumn{2}{c}{ nein } \\
\hline ja & 192 & 37,8 & & 35 & 6,9 \\
nein & 38 & 7,5 & & 228 & 44,9 \\
weiß nicht & 5 & 1,0 & & 10 & 4,0 \\
\hline Summe (N=508) & $\mathbf{2 3 5}$ & $\mathbf{4 6 , 3}$ & & $\mathbf{2 7 3}$ & $\mathbf{5 3 , 7}$ \\
\hline
\end{tabular}

\subsection{Einflussfaktoren auf den Wunsch der Weitereinnahme des stationär erhaltenen} Schlaf- oder Beruhigungsmittels

Wie oben beschrieben, wünschten sich 37,4 \% der Patienten eine Weitereinnahme der stationär verordneten Schlaf- oder Beruhigungsmittel. Das Geschlecht, das Alter, die Vorerfah- 
rungen sowie erlebte Verbesserungen Ein- und Durchschlafstörungen zusammen sowie Nebenwirkungen wurden als potenzielle Einflussfaktoren auf den Wunsch einer Weitereinnahme in einer univariaten und anschließend multivariate logistischen Regression eingeschätzt (Tabelle 9). Die häuslichen Vorerfahrungen mit Schlaf- und Beruhigungsmitteln hatten den größten Einfluss. Im univariaten Modell wünschten Patienten mit Vorerfahrungen zu Hause mit gut vierfach höherer Wahrscheinlichkeit eine weiterführende Einnahme von Schlaf- oder Beruhigungsmitteln (Odds Ratio [OR] = 4,10; $95 \%$-Konfidenzintervall [95\%-KI]: 2,26 - 7,41). Dies bestätigte sich auch im multivariaten Modell (OR = 4,16; $95 \%-K I: 2,10-8,22)$. Subjektiv erlebte Verbesserungen der Ein- und Durchschlafstörungen durch die Einnahme unterstützten ebenfalls signifikant den weiteren Einnahmewunsch. Im multivariaten Modell stach dieser Faktor noch deutlicher hervor.

Einen starken Einfluss schienen zunächst auch die behandelnden Abteilungen zu haben. Im univariaten Modell wünschten sich die Patienten der Geriatrie mit einer über doppelt so hohen Wahrscheinlichkeit eine Weitereinnahme im Vergleich zu Patienten der Chirurgischen Fächer; ähnlich im multivariaten Modell, hier aber nicht mehr signifikant (OR =1,98; 95 \%-KI: $0,91-4,28 ; p=0,083)$. Ebenso ergab das univariate Modell, dass die Zugehörigkeit zur höheren Altersgruppe mit einer halb so großen Wahrscheinlichkeit für den Wunsch einer Weitereinnahme verbunden sei. Dies drehte sich aber im multivariaten Modell in eine gegenteilige Aussage ohne Signifikanz (OR = 2,11; $95 \%-\mathrm{KI}$ : 0,88-5,05; $\mathrm{p}=0,093$ ). Geschlecht und wahrgenommene Nebenwirkungen hatten keinen signifikanten Einfluss auf den Wunsch einer Weitereinnahme (Tabelle 9).

Da die Patienten nur allgemein nach Schlaf- und Beruhigungsmittel befragt wurden bzw. sich nicht unbedingt an die genaue Bezeichnung des eingenommenen Medikaments erinnerten, sollte mit Hilfe der Einträge in der Patientenakte differenziert überprüft werden, ob Patienten, die eine Weitereinnahme wünschten, bestimmte Präparate erhalten hatten (Tabelle 10). Besonders häufig wünschten Patienten, die Z-Substanzen und Baldrianpräparate eingenommen hatten, eine Weitereinnahme; auch im Fall der Benzodiazepine wünschte etwa ein Viertel der Patienten eine weiterführende Behandlung zu Hause. 
Tabelle 9: Einflussfaktoren auf den Wunsch der Weitereinnahme von Schlaf- oder Beruhigungsmitteln nach Krankenhausaufenthalt

\begin{tabular}{|c|c|c|c|c|c|}
\hline \multirow[b]{2}{*}{ Einflussfaktoren } & \multirow[b]{2}{*}{$\%^{*}$} & \multicolumn{2}{|c|}{ univariates Modell } & \multicolumn{2}{|c|}{ multivariates Modell } \\
\hline & & OR $\left(95 \%-K^{\star \star}\right)$ & $\mathbf{p}$ & OR $\left(95 \%-\mathrm{KI}^{\star \star}\right)$ & $\mathbf{p}$ \\
\hline Frauen & 41,2 & 1,0 & & 1,0 & \\
\hline Männer & 31,9 & $0,67(0,38-1,17)$ & 0,157 & $0,86(0,45-1,67)$ & 0,662 \\
\hline Chirurgische Fächer & 26,3 & 1,0 & & 1,0 & \\
\hline Geriatrie & 50,8 & $2,62(1,33-5,15)$ & 0,006 & $1,98(0,91-4,28)$ & 0,083 \\
\hline Innere Medizin & 40,9 & $1,91(0,98-3,73)$ & 0,059 & $2,62(1,21-5,67)$ & 0,014 \\
\hline Keine Einnahme zu Hause & 27,0 & 1,0 & & 1,0 & \\
\hline Einnahme zu Hause & 60,3 & $4,10(2,26-7,41)$ & $<0,0001$ & $4,16(2,10-8,22)$ & $<0,0001$ \\
\hline Junge alte Patienten & 33,7 & 1,0 & & 1,0 & \\
\hline Alte alte Patienten & 58,8 & $0,36(1,17-7,49)$ & 0,005 & $2,11(0,88-5,05)$ & 0,093 \\
\hline $\begin{array}{l}\text { Keine Wahrnehmung von } \\
\text { Verbesserungen der Schlaf- } \\
\text { störungen }\end{array}$ & 11,6 & 1,0 & & 1,0 & \\
\hline $\begin{array}{l}\text { Wahrnehmung von Verbesse- } \\
\text { rungen der Schlafstörungen }\end{array}$ & 43,5 & $5,85(2,20-15,53)$ & 0,0001 & $7,60(2,61-22,08)$ & 0,0002 \\
\hline $\begin{array}{l}\text { Keine Wahrnehmung von } \\
\text { Nebenwirkungen }\end{array}$ & 36,0 & 1,0 & & 1,0 & \\
\hline $\begin{array}{l}\text { Wahrnehmung von Neben- } \\
\text { wirkungen }\end{array}$ & 40,2 & $1,20(0,69-2,10)$ & 0,521 & $1,11(0,59-2,10)$ & 0,749 \\
\hline
\end{tabular}

* gibt jeweils den Anteil der Patienten an, die eine Weitereinnahme nach dem stationären Aufenthalt wünschten ( $N=227)$. Je eine fehlende Angabe zur Abteilungszugehörigkeit und Nebenwirkungen, deshalb $N=226$. Sechs fehlende Angaben für die Einnahme zu Hause, deshalb N=221.

** 95\%- Konfidenzintervall 
Tabelle 10: Wunsch der Weitereinnahme je nach eingenommenem Schlaf- oder Beruhigungsmittel

Wunsch der weiteren Einnahme

\begin{tabular}{lll} 
Einnahme laut Patientenakte von & $\mathbf{n}$ & $\%$ \\
\hline Benzodiazepinen (N=90) & 24 & 26,7 \\
Z-Substanzen (N=49) & 25 & 51,0 \\
Benzodiazepinen und/oder Z-Substanzen (N=132) & 45 & 34,9 \\
Baldrian (N=69) & 33 & 47,8 \\
Mirtazapin (N=62) & 21 & 33,9 \\
\hline
\end{tabular}




\section{Diskussion der Ergebnisse}

Rund $45 \%$ der im Rahmen eines computergestützten Interviews (CAPI) befragten älteren Patienten hatten nach eigener Aussage im Krankenhaus ein Schlaf- oder Beruhigungsmittel erhalten. Laut Patientenakten hatten 29,5\% der interviewten Patienten Medikamente aus der Gruppe der Benzodiazepine oder Z-Substanzen eingenommen. Die Aussagen der Patienten und die Dokumentation in den Patientenakten stimmten sehr gut überein. Fast alle Patienten berichteten von Verbesserungen durch die Einnahme, ca. $40 \%$ aber auch von Nebenwirkungen. Über 30 \% der Patienten wünschten, Schlaf- oder Beruhigungsmittel nach Entlassung aus dem Krankenhaus weitereinzunehmen. Vorerfahrungen mit entsprechenden Medikamenten vor dem Krankenhausaufenthalt sowie die subjektiv erlebten Verbesserungen durch die Einnahme von Schlaf- und Beruhigungsmitteln waren die stärksten Einflussfaktoren für diesen Wunsch der befragten Patienten.

\subsection{Stärken und Schwächen der Studie}

\subsubsection{Persönliche Befragung}

Die Face-to-Face-Befragung eignete sich - trotz einiger Nachteile - am besten für das angestrebte Ziel der Studie, und in dieser Art der Datenerhebung liegt ihre Stärke: Persönliche Befragungen erfordern zwar im Vergleich zu Telefon- oder Internet-Befragungen den höchsten zeitlichen und personellen Aufwand, sind aber sehr flexibel und gestatten eine Anpassung an die Voraussetzungen der Gesprächspartner. Die Möglichkeit, Patienten kurz vor ihrer Entlassung aus dem Krankenhaus zu befragen und innen die Gelegenheit einzuräumen, bei Unklarheiten gegebenenfalls nachzufragen, ist ein entscheidender Vorteil des persönlichen Interviews (Scholl 2015). So konnte in der besonderen stationären Situation auf die individuellen Bedürfnisse der Patienten Rücksicht genommen werden. Im Ergebnis sorgte dies Vorgehen für eine hohe Akzeptanz der Befragung und eine sehr große Anzahl vollständiger für die Studie verwertbarer Antworten. Gleichzeitig konnten die Interviewerinnen durch persönlichen Kontakt zur Beantwortung aller Fragen motivieren und die Abbrüche trotz der Länge der Befragung verhindern.

Die Beeinflussung der Ergebnisse durch einen Interviewer-Bias, also eine mögliche Verzerrung der Antworten durch den sozialen, persönlichen Kontext während der Befragung sowie ein spezifisches Rollenverhältnis zwischen Befragten und Interviewer ist nicht auszuschlieBen (Albers et al. 2007). Aussehen, Geschlecht und Alter, Kleidung, die persönliche Erscheinung und andere individuelle Merkmale des Interviewers beeinflussen die Interaktion und damit das Antwortverhalten während einer Face-to-Face-Befragung. Um diesen Effekt zu 
reduzieren, achteten die beiden Interviewerinnen auf eine strikte Standardisierung und ein gleichermaßen neutrales, gepflegtes Aussehen im weißen medizinischen Kittel.

Die persönliche Befragung birgt die Gefahr, dass Studienteilnehmer die vom Interviewer erwarteten Antworten geben (im Sinne einer „sozialen Erwünschtheit“). Die Interviewerinnen wiesen aus diesem Grund in ihrer einleitenden Vorstellung bewusst darauf hin, dass sie nicht zum medizinischen Personal der Station gehörten und die Befragung zwar im Evangelischen Krankenhaus Göttingen-Weende, aber nicht durch das Stationspersonal und im Auftrag des Krankenhauses durchgeführt würde. Für alle Studienteilnehmer sollte ersichtlich sein, dass die Befragung keinerlei Einfluss auf ihre stationäre Behandlung hätte und die medizinischen Mitarbeiter keine Einsicht in die Antworten erhalten würden. Auch deswegen wurde die Befragung bewusst erst kurz vor Entlassung der Patienten durchgeführt. Um weitere Verunsicherungen zu vermeiden, wurden alle Studienteilnehmer auf die Unabhängigkeit der Befragung, die Möglichkeit, sie jederzeit abbrechen oder auch Fragen nicht beantworten zu können sowie die Wahrung der Anonymität und die Verschwiegenheit der Interviewerinnen hingewiesen. Durch diese standardisierte Vorgehensweise in der Einführung der Befragung sollte eine Verzerrung der Ergebnisse minimiert werden, wenn sie auch nicht gänzlich ausgeschlossen werden kann (Albers et al. 2007; Scholl 2015).

Computerunterstützt konnte die Befragung schnell und präzise ausgeführt werden. Antworten der Patienten wurden direkt in den Computer eingegeben; fehlende Werte (sogenannte missings) wurden durch eine klare Struktur und Organisation des elektronischen Fragebogens weitgehend vermieden.

Die Reliabilität der Antworten konnte nicht überprüft werden, da das Studiendesign eine Zweitbefragung der Patienten oder vergleichbare Verfahren nicht vorsah und dies auch nicht praktikabel gewesen wäre.

\subsubsection{Teilnahme und Stichprobe}

Mit einer Teilnahme von 73 \% ist die Ausschöpfung der Stichprobe als sehr gut zu bewerten (Baruch und Holtom 2008). Befördert wurde dies sicher durch die aufsuchende Befragung, das persönliche Ansprechen und Motivieren der Patienten durch die zwei Interviewerinnen. Allerdings stellte die Befragung am Morgen sowie kurz vor der Entlassung aus dem Krankenhaus eine besondere Herausforderung dar. Eine Teilnahmeverweigerung erfolgte am häufigsten aus zwei Gründen:

- $\quad$ Die Patienten waren schon bereit für die stationäre Entlassung und warteten z. B. nur noch auf ihre Angehörigen. In dieser Situation äußerten einige potenzielle Teilnehmer, dass sie sich angespannt fühlten und lehnten daher die Teilnahme an der Befragung $a b$. 
- $\quad$ Einige Patienten fühlten sich verunsichert, weil sie die Einverständniserklärung zur Teilnahme an der Studie und zur Nutzung der Daten unterschreiben sollten. Diese ursprünglich als Absicherung für die Patienten gedachte Maßnahme mobilisierte negative Assoziationen und ließ manche Patienten unlautere Absichten vermuten - trotz der ausführlichen Erklärung zur Studie, deren Ziel und der Rechtslage.

Genauere Informationen zu den Patienten, die einer Befragung nicht zustimmten, wurden nicht erhoben. Dies ist eine Schwäche der Studie.

Die Befragung von 508 Patienten bietet einen guten Querschnitt der älteren Klientel eines Krankenhauses der Grund- und Regelversorgung. Knapp $50 \%$ der Patienten kamen von den Stationen der chirurgischen Fachbereiche und je $25 \%$ aus der Inneren Medizin und der Geriatrie. Der hohe Anteil der chirurgischen Patienten lässt sich durch die Anzahl der entsprechenden Stationen sowie eine kürzere Liegedauer im Vergleich zu den anderen beiden Fachbereichen erklären. Auf der Station der Plastischen Chirurgie konnten nur 11 Patienten und auf der Station der Hals-Nasen-Ohren-Heilkunde keine Patienten befragt werden. Gründe können ebenfalls in der kurzen Liegedauer, einer jüngeren Patientenpopulation oder in organisatorischen Problemen liegen.

Die Stichprobe kann mit den Diagnosedaten des Statistischen Bundesamtes verglichen werden. Demzufolge waren 2014 43,2\% der Patienten im Krankenhaus über 65 Jahre, davon waren 46,4 \% Männer und 53,6 \% Frauen (Statistisches Bundesamt 2015). Der Anteil der älteren Patienten im Evangelischen Krankenhaus Göttingen-Weende war im selben Zeitraum mit 54,3\% höher als im deutschlandweiten Vergleich. Die Stichprobe dieser Studie repräsentiert mit 40,9\% männlichen und 59,1\% weiblichen Teilnehmenden die Belegung eines durchschnittlichen deutschen Krankenhauses nicht perfekt, ist aber akzeptabel. Erklärungsansätze für diese Abweichungen könnten folgende sein: Zum einen verfügt das Evangelische Krankenhaus Göttingen-Weende nicht über bestimmte Fachabteilungen, wie z.B. einer psychiatrischen oder einer gynäkologischen Klinik. Zum anderen kann angenommen werden, dass Patienten mit speziellen oder besonders schwerwiegenden Krankheitsbildern eher in der direkt angrenzenden Universitätsklinik behandelt werden. Methodisch muss zusätzlich bedacht werden, dass die Teilnahme an der Studie freiwillig war und Patienten auch die Teilnahme verweigern konnten. Außerdem mussten die oben aufgeführten Kriterien für den Einschluss an der Studie erfült sein. So blieben - wie beschrieben - u. a. demenziell erkrankte Patienten außer Acht. Dies könnten Gründe sein, warum die Stichprobe leichte Abweichungen von einem durchschnittlichen deutschen Krankenhaus aufweist.

\subsubsection{Demenz und Orientierungslosigkeit als Ausschlusskriterien}

Die Bedingung, wonach die Patienten klar und orientiert zum Ort und zur Zeit sein mussten, schließt eine wichtige Patientengruppe aus, die vermutlich einen hohen Gebrauch an Schlaf- 
und Beruhigungsmitteln hat. Speziell von dementen Patienten höheren Alters ist bekannt, dass sie einen vermehrten Gebrauch an Hypnotika haben (Giron et al. 2001). Da aber verlässliche Antworten bei vorliegender Demenz als Grunderkrankung nicht gewährleisten werden können, wurden diese Patienten nicht befragt. Demzufolge ist aber sehr wahrscheinlich, dass der Gebrauch von Schlaf- und Beruhigungsmitteln eher unterschätzt wurde.

\subsubsection{Berücksichtigung spezifischer Stoffklassen}

Eine weitere Limitation ist, dass Patienten pauschal nach Schlaf- und Beruhigungsmitteln befragt wurden, nicht aber spezifisch nach den im Fokus der Studie liegenden Stoffklassen der Benzodiazepine und Z-Substanzen. Nur unter Berücksichtigung dieses Umstandes können die Ergebnisse mit der internationalen Forschung, die sich insbesondere mit Benzodiazepin- und Z-Substanz-Hypnotika beschäftigt, verglichen werden. Diese Einschränkung ist zugleich eine Stärke der Studie, da somit ein umfassendes Bild der Verordnung aller in Frage stehender Schlaf- und Beruhigungsmittel aus Sicht der Patienten im Krankenhaus entstand.

Nicht auszuschließen ist, dass Patienten bestimmte Arzneimittel - zum Beispiel Schmerzmedikamente - fälschlicherweise für Schlaf- und Beruhigungsmittel hielten. Um diese mögliche Fehlerquelle zu überprüfen und die Prävalenzen der einzelnen Stoffklassen nachvollziehen zu können, wurden die Antworten der Patienten aus der Befragung mit den Daten in den Patientenakten verglichen.

\subsubsection{Indifferente Antworten der Patienten}

Bei mehreren Fragen hatten die Patienten die Option, mit „weiß nicht“ zu antworten. Überraschenderweise wussten 15 Patienten nicht, ob sie Schlaf- oder Beruhigungsmittel während ihres Krankenhausaufenthalts eingenommen hatten oder nicht. Ebenso konnten 7 Patienten nicht eindeutig beantworten, ob sie zu Hause ein solches Medikament schon einmal eingenommen hatten. Gleichzeitig wusste nur ein Viertel der Patienten, welches Medikament sie im Krankenhaus einnahmen. Auch wenn es sich dabei nur um wenige Patienten handelt, ist es dennoch beunruhigend und Anlass zu weiterer Nachforschung, dass zumindest ein Teil der Patienten - wie in Hypothese 3 angenommen - sich nicht gut über ihre stationäre Medikation informiert fühlt oder sich nicht danach erkundigt. Gerade bei der Einnahme von Schlaf- und Beruhigungsmitteln würde man erwarten, dass Patienten diese auf expliziten Wunsch oder nach der Angabe von gestörtem Schlaf im Krankenhaus erhalten. In mehreren Studien wurde das Problem untersucht, mit dem Ergebnis, dass Patienten wenig über ihre stationär verschriebenen Medikamente wussten und auch die Indikation der medikamentösen Therapie ihnen nicht bekannt war (Pullar et al. 1989; Kerzman et al. 2005; Makaryus und Friedman 2005). Dies zeigt den Bedarf einer Patientenedukation insbesondere auch bei der Verordnung von Hypnotika und Sedativa, die - wie beschrieben - gerade bei älteren Patien- 
ten durch kognitive Einbußen zu Stürzen und anderen schwerwiegenden Nebenwirkungen führen können. Die Wirksamkeit einer solchen Patientenschulung und die Beteiligung der Patienten an der medikamentösen Behandlung wurde bereits in Studien bestätigt (Lowe et al. 1995; Sirdifield et al. 2017).

\subsection{Einnahmeprävalenz während des stationären Aufenthalts}

Immerhin 44,7\% der befragten Patienten hatten laut eigener Aussage ein Schlaf- oder Beruhigungsmittel während des Krankenhausaufenthalts eingenommen; laut Patientenakte erhielten knapp $30 \%$ ein Benzodiazepin oder eine Z-Substanz. So hat fast jeder zweite entsprechend der Hypothese 1 stationär ein Schlaf- oder Beruhigungsmittel erhalten.

Die Einnahmeprävalenz liegt im oberen Bereich der internationalen Studien, mit Prävalenzen bei Benzodiazepin- und Z-Substanz-Hypnotika zwischen $20 \%$ und $45 \%$. Diese Daten stammen allerdings in der Regel aus Auswertungen von Patientenakten. So erhielten $21 \%$ der Patienten eines britischen Lehrkrankenhauses Hypnotika und Anxiolytika; in $80 \%$ der Fälle handelte es sich laut Krankenhausakte um Benzodiazepine (Edwards et al. 1991). In einer japanischen Studie eines Universitätsklinikums lag die Prävalenz von Benzodiazepinen bei $23 \%$ für ältere Patienten (Nakao et al. 2009). Noch höher lagen die Raten bei einer kanadischen und einer niederländischen Studie. Hier lag die Prävalenz der Einnahme von Hypnotika bzw. einer Schlafmedikation bei je 34 \% (O'Reilly und Rusnak 1990; Halfens et al. 1991). Gemäß einer US-amerikanische Studie (Zisselman et al. 1994) und einer belgischen Studie (Somers et al. 2011) sowie deren Vorgängerstudie im selben Universitätskrankenhaus (Warie et al. 2003) hatten über $40 \%$ der Patienten Schlafmittel eingenommen. Allerdings wurden in den meisten Studien ausschließlich Benzodiazepine erhoben - mit wenigen Ausnahmen (Halfens et al. 1991; Warie et al. 2003; Somers et al. 2011). Unsere Studie hat dagegen alle verordneten Schlaf- und Beruhigungsmittel miteinbezogen und somit mehrere Medikamentengruppen untersucht. Die Einnahme laut der Studie von Warie et al. (2003) war mit $45 \%$ ähnlich unserer Studie mit 44,7\% (Patientenaussagen) bzw. von 46,3\% (Patientenakten). Auch der Anteil der Benzodiazepine und Z-Substanzen mit einer Prävalenz von knapp $30 \%$ ist vergleichbar mit anderen Studien.

Nur gut $25 \%$ der Patienten konnten benennen, welches Medikament sie während des stationären Aufenthalts verordnet bekommen hatten. Diesen Mangel an Kenntnis über die eigene Schlafmedikation beschrieben auch Anthierens et al. (2007). Dies gilt laut einer belgischen Studie insbesondere für ältere Patienten im Krankenhaus und nicht nur speziell für Schlafund Beruhigungsmittel. So wussten ältere Patienten weniger über die Medikamente, die sie stationär erhielten, als jüngere Patienten (Breemhaar et al. 1990). 
Viele Patienten berichteten von einer Verbesserung ihrer Ein- und Durchschlafstörungen nach der Einnahme der Schlaf- oder Beruhigungsmittel, selten hingegen von einer Verbesserung bestehender Angststörungen. Auch in anderen Studien waren die Hauptgründe für eine Einnahme die Insomnien, die sich für ca. $80 \%$ der befragten Patienten dadurch verbesserten (King et al. 1990; Siriwardena et al. 2008).

Die Motive für eine Einnahme im Krankenhaus scheinen sich von denen zu Hause zu unterscheiden. So gelten im Krankenhaus erlebte Schlafstörungen als Hauptindikationen für die Verordnung von Schlaf- und Beruhigungsmittel. Anders als im ambulanten Bereich nimmt die Behandlung von Angststörungen durch die anxiolytische Wirkung insbesondere von Benzodiazepinen und Z-Substanzen einen geringeren Stellenwert ein. Dies zeigt auch die Studie von Siriwardena et al. (2008). Hier wurden Angststörungen als Indikation für die Einnahme im Krankenhaus ähnlich unserer Studie seltener angegeben als Insomnien. Studien über die ambulante Behandlung hingegen zeigten, dass Angst einer der Hauptgründe für die Langzeiteinnahme von Hypnotika ist (King et al. 1990; Barter und Cormack 1996; Cook et al. 2007).

Von Nebenwirkungen durch die Einnahme von Schlaf- und Beruhigungsmittel berichteten Patienten auffällig häufig, nämlich fast $40 \%$. Die Hypothese 4, wonach die Kognition, Psychomotorik und Vigilanz durch die Einnahme von Schlaf- und Beruhigungsmittel (insbesondere durch Hypnotika) negativ beeinflusst werden, bestätigte sich im Erleben der Befragten. Da es sich in dieser Studie nicht um eine explizite Medikamentenstudie handelte, dürfen keine direkten Schlüsse aus den Äußerungen der Patienten gezogen werden; allerdings sind u.a. die zwei am häufigsten genannten Nebenwirkungen - verlängerte Schläfrigkeit und Benommenheit - für Benzodiazepine und Z-Substanzen typisch und bekannt (King et al. 1990; Glass et al. 2005; Siriwardena et al. 2008). Im Vergleich zu anderen Befragungen, in denen Nebenwirkungen in $16 \%$ (King et al. 1990) bzw. über $40 \%$ (Siriwardena et al. 2008) wahrgenommen worden waren, liegt das Ergebnis dieser Studie im oberen Bereich. Konzentrationsschwierigkeiten und Kopfschmerzen als negative Auswirkungen waren in dieser Befragung dagegen seltener als in den anderen Studien wahrgenommen worden.

Auswirkungen auf die Motorik erwähnten zwar nur wenige Patienten, aber immerhin führten 13 Patienten Gangunsicherheiten und sogar 3 Patienten Stürze auf ihre Einnahme von Schlaf- und Beruhigungsmittel zurück. Diese Nebenwirkungen gehören zu den gefährlichsten unerwünschten Wirkungen speziell bei älteren Patienten. Ältere Menschen haben möglicherweise aufgrund von anderen alters- oder krankheitsbedingten Gebrechen bereits lokomotorische Einschränkungen. Eine erhöhte Frakturgefahr besteht bei der Einnahme von motorisch beeinflussenden Medikamenten und ist mehrfach insbesondere für Benzodiazepine 
und Z-Substanzen beschrieben (Ray et al. 1989; Wang et al. 2001a; Wang et al. 2001b; Wagner et al. 2004; Finkle et al. 2011; Gunja 2013; Yu et al. 2017).

Vielfach diskutiert werden in der Literatur kognitive Einschränkungen und - besonders kontrovers - die negative Beeinflussung von Demenzerkrankungen durch die Einnahme von Benzodiazepinen oder Z-Substanzen (Lagnaoui et al. 2002; Puustinen et al. 2007; Mets et al. 2011; Billioti de Gage et al. 2012; Billioti de Gage et al. 2014). Immerhin 7 Patienten dieser Studie fühlten sich durch die Einnahme der Schlaf- oder Beruhigungsmittel vergesslicher und hatten subjektiv eine Beeinflussung des Erinnerungsvermögens wahrgenommen. Damit können keineswegs valide Schlussfolgerungen in Richtung der Demenz-Hypothese gezogen werden.

Die Einnahmefrequenz der Schlaf- oder Beruhigungsmittel der Patienten im Krankenhaus wurde meist als „täglich“ angegeben. Dies widerspricht für den begrenzten Zeitraum im Krankenhaus nicht den Empfehlungen der einschlägigen Leitlinien. Die kurzzeitige Einnahme birgt aber - insbesondere bei älteren und krankheitsbelasteten Patienten - bereits das Risiko der oben beschriebenen Nebenwirkungen, zum Beispiel Gangunsicherheit, Benommenheit bis hin zu Stürzen. Die Verordnung von Schlaf- oder Beruhigungsmitteln im Krankenhaus könnte außerdem Einfluss auf die Patienten und ihre Risikobewertung der Medikamente haben. Patienten bekommen die Schlaf- oder Beruhigungsmittel von Ärzten stationär angeboten und verordnet. Es könnte der Eindruck entstehen, dass die Einnahme der Arzneimittel von den verschreibenden Experten als unproblematisch eingeschätzt wird. Patienten hatten die Schlaf- oder Beruhigungsmittel trotz Nebenwirkungen zum Großteil als positiv beurteilt. Diese Bewertung und das mögliche Sicherheitsgefühl - vermittelt durch die Ärzte im Krankenhaus - könnten erklären, warum über $30 \%$ der Patienten sich eine Weitereinnahme zu Hause wünschten.

In einer amerikanischen Studie wurde darauf hingewiesen, dass Patienten dazu neigen, Nebenwirkungen zu verneinen oder zu bagatellisieren (Cook et al. 2007). Aufgrund des Interviewer-Bias (siehe 6.1.1) unserer Befragung könnten die wirklich wahrgenommenen Nebenwirkungen durch diese Befragung unterschätzt worden sein.

\subsection{Geschlechtsspezifische Unterschiede}

Dass sich die Prävalenz in der Einnahme von Schlaf- und Beruhigungsmittel zwischen Männern und Frauen nicht unterscheidet, überrascht und widerspricht der Hypothese 2. In den meisten Studien überwiegt die Einnahme von Schlaf- und Beruhigungsmitteln durch Frauen (Edwards et al. 1991; Gleason et al. 1998; Warie et al. 2003; Petitjean et al. 2007; Nakao et al. 2009; Sonnenberg et al. 2012; Glaeske et al. 2013; Schlack et al. 2013; Hoffmann und Glaeske 2014). Edwards et al., Warie et al. und Nakao et al. beziehen sich auf den Kranken- 
hausaufenthalt; die Tendenz gilt aber auch für den ambulanten Bereich. Drei ältere Studien, die keinen Einfluss des Geschlechts auf die Einnahme von Schlaf- und Beruhigungsmitteln feststellten, kommentierten oder erklärten diese Besonderheit nicht (O'Reilly und Rusnak 1990; Noble et al. 1993; Zisselman et al. 1994).. Auch das „Jahrbuch Sucht 2017“ beschreibt die Problematik der Suchtabhängigkeit von Schlaf- und Beruhigungsmitteln als vorwiegend weibliches Problem (Deutsche Hauptstelle für Suchtfragen e.V. 2017). Zwei qualitative Studien zum Langzeitgebrauch von Benzodiazepinhypnotika warnen allerdings vor dem stereotypen Bild der älteren Frau, die unbesorgt Schlafmittel einnimmt. Vielmehr handele es sich bei den Konsumenten der Benzodiazepine um ein inhomogenes Patientenkollektiv (King et al. 1990; Barter und Cormack 1996). Dies zeigte sich auch in den Ergebnissen unserer Studie und widerspricht den klischeehaften Vorstellungen über Schlaf- und Beruhigungsmittel leichtfertig konsumierende Frauen (und Männer).

\subsection{Relevanz der Vorerfahrungen mit Schlaf- und Beruhigungsmitteln}

Knapp $20 \%$ der befragten Patienten hatten häusliche Vorerfahrungen mit Schlaf- und Beruhigungsmitteln, mehr Frauen als Männer. Dies entspricht den oben dargestellten Erkenntnissen in der Fachliteratur, von der die ambulante Einnahme insbesondere von Benzodiazepinen bei älteren Menschen auf 10 bis 20 \% geschätzt wird (Gleason et al. 1998; Petitjean et al. 2007; Glaeske et al. 2013; Johnson et al. 2016). Gleason et al. (1998) hatten außerdem ermittelt, dass etwa $40 \%$ der älteren Patienten freiverkäufliche Schlaf- oder Beruhigungsmittel einnehmen. In aktuelleren Studien lag die ambulante Einnahme alternativer Schlafmittel im Bereich von 10 bis 25 \% (Johnson et al. 1998; Stasio et al. 2008; Lund et al. 2010). Die Nutzung dieser Mittel wurde in unserer Studie miteinbezogen, was die recht hohe Prävalenz mit erklären mag. Dass über $80 \%$ Patienten, die vor einem Klinikaufenthalt bereits Schlafoder Beruhigungsmitteln eingenommen haben, diese dann auch im Krankenhaus benötigen, ist nicht überraschend und bereits aus anderen Untersuchungen bekannt (Warie et al. 2003; Somers et al. 2011). Dass aber gut $30 \%$ der Patienten die Schlaf- oder Beruhigungsmittel erstmalig im Rahmen des aktuellen Krankenhausaufenthalts einnahmen, gibt hingegen Anlass zur kritischen Prüfung des Medikamentenkonsums in Krankenhäusern, zumal diese Tendenz sich auch in anderen Studien andeutet (Edwards et al. 1991; Zisselman et al. 1994; Lagnaoui et al. 2001).

Ähnlich der Studie von Siriwardena et al. (2008), hatte auch in dieser Studie ein Teil der Patienten Schlaf- oder Beruhigungsmittel über einen langen Zeitraum eingenommen und viele hatten bereits versucht, sich von den Medikamenten zu entwöhnen. Patienten scheinen für das Abhängigkeitspotenzial ihrer Schlafmedikation sensibler zu sein, nehmen sie aber dennoch nach gescheiterten Absetzversuchen über Jahre ein (Barter und Cormack 1996). 


\subsection{Wunsch der weiteren Einnahme nach Entlassung}

Obwohl erlebte Nebenwirkungen von Patienten in der Befragung häufig thematisiert wurden, bewerteten über $80 \%$ der Patienten die eingenommenen Schlaf- oder Beruhigungsmittel als insgesamt eher positiv und über $37 \%$ wünschten - der fünften Hypothese entsprechend eine weiterführende Einnahme. Auch in anderen Studien hatten Patienten nach den Erfahrungen im Krankenhaus die Behandlung mit den erhaltenden Schlaf- oder Beruhigungsmittel fortsetzen wollen (Warie et al. 2003; Siriwardena et al. 2008). Vorerfahrungen und wahrgenommene Verbesserungen fördern offensichtlich diesen Wunsch. Eine Kontinuität in der Behandlung von Insomnien mit Schlaf- und Beruhigungsmittel wird demzufolge - selbst nach erlebten, einschränkenden Nebenwirkungen - bevorzugt. Dies mag, wie oben angedeutet, mit der Tendenz von Patienten zusammenhängen, negativen Begleiterscheinungen von eingenommenen Schlaf- und Beruhigungsmitteln zu verneinen bzw. zu bagatellisieren, um nicht eine Reduktion der Medikation in Kauf nehmen zu müssen (Cook et al. 2007).

\subsection{Freiverkäufliche und ärztlich verordnete Medikamente}

Baldrianpräparate waren nach den Benzodiazepinen die am zweithäufigsten eingenommenen Arzneimittel bei Schlafstörungen. Bekannt ist, dass Menschen häufig freiverkäufliche Medikamente gegen auftretende Schlafprobleme einnehmen, vermehrt auch zusätzlich zu anderen Schlaf- oder Beruhigungsmitteln (Gleason et al. 1998). Die Rolle dieser Arzneimittel in der stationären Versorgung ist bisher nicht gut erforscht. Unsere Befragung unterstreicht jedoch die Relevanz dieses Themas, insbesondere für ältere Menschen.

Ebenfalls von großer Bedeutung ist der Wunsch nach Weiterführung der Einnahme von Schlaf- und Beruhigungsmittel im häuslichen Umfeld. Wurden pflanzliche Medikamente im Krankenhaus gegeben, wünschten $48 \%$ der Patienten eine Weitereinnahme zu Hause. In Anbetracht der geringen Nebenwirkungen dieser Präparate ist dieses Ergebnis akzeptabel.

Überraschend ist aber, dass $51 \%$ Patienten, die ein Medikament aus der Klasse der ZSubstanzen eingenommen hatten, sich ebenfalls eine weiterführende Behandlung wünschten. Immerhin traf dies auch für knapp $27 \%$ der Patienten zu, die ein Benzodiazepin erhalten hatten. Siriwardena et al. (2008) hatten in ihrer Studie diesen Unterschied zwischen der Einnahme von Benzodiazepinen und Z-Substanzen nicht feststellen können. So wollte die Mehrheit beider Patientengruppen die Einnahme weiterführen; Patienten der BenzodiazepinGruppe sogar etwas häufiger. Ob in unserer Studie die Substanzen selbst oder aber andere Faktoren für die Unterschiede zwischen Benzodiazepinen und Z-Substanzen eine Rolle spielten, ist nicht zu entscheiden, würde zur Klärung weiterführende Untersuchungen bedürfen. 


\subsection{Differenzen zwischen den Fachabteilungen}

Es zeigten sich Unterschiede zwischen den Fachabteilungen des Krankenhauses. So antworteten in der Geriatrie deutlich mehr Patienten, Schlaf- oder Beruhigungsmittel eingenommen zu haben als in der Inneren Medizin oder den chirurgischen Fächer. In der Geriatrie war erwartungsgemäß auch das Durchschnittsalter der Patienten besonders hoch. Da bekannt ist, dass mit steigendem Alter die Prävalenz von Schlafstörungen und der Bedarf an Schlaf- und Beruhigungsmittel wachsen, könnte dies die höhere Einnahme in der Geriatrie erklären (O'Reilly und Rusnak 1990; Zisselman et al. 1994; Gleason et al. 1998; Ohayon 2002; Ancoli-Israel 2005; Petitjean et al. 2007; Ancoli-Israel 2009; Nakao et al. 2009; Glaeske et al. 2013).

Außerdem unterscheiden sich die geriatrischen Behandlungsansätze von denen der anderen Abteilungen: Der Fokus in der Geriatrie liegt auf der Risikoerkennung von Krankheiten, Prävention und (Früh-) Rehabilitation. Zu den Therapiekonzepten gehören u.a. die umfangreiche Diagnostik alterstypischer Krankheiten (beispielsweise dementieller Erkrankungen) und die Prüfung der aktuellen Medikation inklusive einer Anpassung und Neueinstellung (Bruder et al. 1991). Aus diesen Gründen und insbesondere im Rahmen einer Frührehabilitation ist der Aufenthalt der Patienten auf den Stationen der Geriatrie durchschnittlich länger als auf anderen Stationen. Diese Tatsache in Verbindung zu der ungewohnten Umgebung kann vermehrt zu Störungen des Schlafes und zu Unruhe führen, die eine medikamentöse Behandlung nahelegen (O’Reilly und Rusnak 1990).

\subsection{Verlässlichkeit der Patientenaussagen im Vergleich zu den Angaben in den Pa- tientenakten}

Der Vergleich der Aussagen der Patienten zur Einnahme von Schlaf- oder Beruhigungsmitteln mit der Dokumentation in der Patientenakte zeigte eine hohe Übereinstimmung. Die dokumentierte Einnahme von Schlaf- oder Beruhigungsmitteln lag nur geringfügig höher als gemäß Angaben der Patienten. Dies kann daran liegen, dass 15 Patienten auf die Frage einer Verordnung im Krankenhaus mit „weiß nicht“ antworteten. Denkbar ist auch, dass die Patientenakten nicht immer vollständig sind oder Patienten bestimmte Medikamente aus privatem Bestand parallel einnahmen - ohne Dokumentation in der Patientenakte. Die Studie von Somers et al. (2011) kam zu einem ähnlichen Ergebnis. Hier lag die Einnahme von Hypnosedativa laut der Dokumentation um 10 \% höher als gemäß Angaben der Patienten. Cohen's Kappa von 0,70, das nach der Einteilung von Landis und Koch als „substantial“ gilt und eine beachtliche Reliabilität bestätigt, unterstreicht die Verlässlichkeit der Patientenangaben (Fleiss und Cohen 1973; Landis und Koch 1977). 
Dieses Ergebnis überrascht, da mehrere Studien gezeigt haben, dass die Angaben aus Patientenbefragungen häufig stark - bis zu über $80 \%$ - von der Aktendokumentation abweichen, insbesondere bei älteren Patienten. So zeigte eine Studie über die Medikamenteneinnahme von geriatrischen Patienten im Vergleich zur Dokumentation in hausärztlichen Akten, dass $86,7 \%$ der Aussagen nicht mit den Angaben der Akten übereinstimmten (Tulner et al. 2009). Bestätigt wurde dies ebenso in der Studie von Bedell et al. (2000), in der 76 \% der Aussagen der Patienten sich nicht in den Patientenakten wiederfanden. Hier waren allein $51 \%$ der angegebenen Einnahmen nicht dokumentiert und 29 \% der Patienten nahmen Medikamente, die in der Akte aufgeführt waren, gar nicht ein. Großen Einfluss auf die fehlende Übereinstimmung hatte hier insbesondere ein höheres Alter der Patienten. Zu anderen Ergebnissen kommt aber eine deutsche Studie. Darin konnten über 600 Patienten aus Hausarztpraxen zu Dosis, Indikation und Einnahmeschema ihrer Hausmedikation verlässlich Auskunft geben. Insbesondere kannten $85 \%$ der Patienten die Indikation ihrer verschriebenen Arzneimittel. Nur in je ca. $10 \%$ war die Medikation nur in der Patientenakte dokumentiert oder ausschließlich eine Angabe des Patienten (Chaudhuri et al. 2011).

Andererseits finden sich Hinweise in der Fachliteratur, dass Patienteninterviews in Kombination mit einer Datenerhebung aus den Patientenakten methodisch geeignet sind, um Erkenntnisse über Medikamenteneinnahmen zu gewinnen (Sjahid et al. 1998). So kann die für diese Studie gewählte Methodik als sehr zweckmäßig eingeschätzt werden. Trotz der sich bestätigenden Erwartung, dass Patienten schlecht über ihre spezifische Medikation informiert seien, zeigte sich eine hohe Verlässlichkeit der Patientenaussagen - zumindest was das Faktum der Einnahme selbst anbelangt. 


\section{Ausblick}

Die Ergebnisse dieser Studie zeigen, dass ältere Patienten im Krankenhaus häufig Schlafund Beruhigungsmittel einnehmen und dabei Verbesserungen, aber auch Nebenwirkungen wahrnehmen. Beunruhigend ist nicht nur die große Zahl von Patienten, die zumindest einmal im Krankenhaus Schlaf- oder Beruhigungsmittel erhalten haben; viele von ihnen würden gern vergleichbare Arzneimittel auch zu Hause einnehmen bzw. verschrieben bekommen. Das Geschehen im Krankenhaus strahlt also auch auf die ambulante Versorgung aus.

Die Sensibilität älterer Patienten gegenüber den potenziell gefährlichen Nebenwirkungen von Schlaf- und Beruhigungsmittel, ihre speziellen durch das Alter bedingten Bedürfnisse und körperlichen Konstitutionen sowie die Gegebenheiten in einem Krankenhaus und im häuslichen Bereich der Patienten sollten in weiterführende Überlegungen für eine Intervention einbezogen werden. Bekannt ist auch, dass Krankenhausärzte und Pflegepersonal Benzodiazepine und Z-Substanzen in ihrer Verwendung und im Nutzen-Schaden-Verhältnis unterschiedlich einschätzen, ebenso Hausärzte und Apotheker (Hoffmann 2013; Heinemann u. Himmel 2017). Eine Intervention zur Verbesserung des Umgangs mit Schlaf- und Beruhigungsmitteln sollte sich deswegen an alle beteiligten Akteure richten und demzufolge Patienten, Pflegepersonal sowie Haus- und Krankenhausärzte einbeziehen und deren Kommunikation und Abstimmung untereinander verbessern.

Krankenhausärzte, Pflegepersonal, Hausärzte und die Patienten selber sollten intensiver beteiligt werden, um durch Sensibilisierung und vermehrte Achtsamkeit die Einnahme von Benzodiazepinen und Z-Substanzen bei älteren Patienten senken und das Risiko für Stürze, Knochenbrüche, Verwirrtheit und Abhängigkeit reduzieren zu können.

Unter Einbezug aller Forschungsergebnisse aus dem Gesamtprojekt wird im Evangelischen Krankenhaus Göttingen-Weende ein „schlaffreundliches Krankenhaus“ pilotiert. Beabsichtigt ist, die individuelle Beratung und ggf. Behandlung jedes stationären Patienten einem Algorithmus folgen zu lassen. Dieser beinhaltet zunächst eine Einstufung des Patienten mit Hilfe einer Schlafanamnese bei Krankenhausaufnahme. Daraufhin folgt gegebenenfalls die adäquate ärztliche Verordnung eines Schlafmittels mit Handlungsstrategien für Pflege und Ärzte im Umgang mit akuten Schlafproblemen eines Patienten während des stationären Aufenthalts; alternative Möglichkeiten der Schlafanstoßung neben medikamentöser Behandlung sind inkludiert. Die Empfehlungen gehen im Speziellen auf die Aufgaben und Handlungsmöglichkeiten von Pflege und ärztlichem Team ein und beziehen die Hinweise zur medikamentösen Behandlung von Schlafstörungen für ältere Patienten der PRISCUS-Liste ein. 
Soweit Wünsche zur Fortsetzung der im Krankenhaus erhaltenen Medikation an Hausärzte herangetragen werden, wäre eine wesentliche Aufgabe, besser über die Wirkungen und Nebenwirkungen sowie die Folgen einer langen Einnahme aufzuklären und Patienten Alternativen zu Benzodiazepinen und Z-Substanzen - den Leitlinien folgend - anzuraten und deren Anwendung detailliert zu besprechen. Da vermehrt psychische Belastungen und Angststörungen Auslöser für langfristige Insomnien im Alter sind und Patienten diese auch entsprechend beschreiben, sollte der Schwerpunkt der Behandlung auf kognitivverhaltenstherapeutischen Methoden liegen, wie es die S3-Leitlinie empfiehlt, und damit weniger auf der medikamentösen Behandlung. In diesem Fall wird der Patient aktiv in die Verbesserung des eigenen Schlafverhaltens einbezogen und kann sich selbst helfen, zum Beispiel indem der Theorie der Schlafhygiene gefolgt wird. In diesem Bereich besteht weiterer Forschungsbedarf, um die aufwändigen Methoden sowohl im Krankenhaus als auch im häuslichen Bereich integrieren zu können.

Das zum Zeitpunkt des Abschlusses dieser Arbeit veröffentlichte „Jahrbuch Sucht 2017“ betont das Problem der weitverbreiteten Medikamentensucht. In der Altersgruppe über 65 Jahren seien insbesondere ältere Frauen von einer Schlaf- und Beruhigungsmittelabhängigkeit betroffen (Deutsche Hauptstelle für Suchtfragen e.V. 2017). So ist das Ziel, Patienten mit Hilfe adäquat kurzfristiger Medikation und Intervention - möglichst unter Verzicht von Hypnosedativa - bei ihren Schlafproblemen Erleichterung zu verschaffen, aktuell umso bedeutsamer. 


\section{Zusammenfassung}

Hintergrund. Schlaf- und Beruhigungsmittel kommen gerade in der speziellen Situation eines Krankenhausaufenthalts häufig zum Einsatz, oft sind es Benzodiazepine und ZSubstanzen. Ihre Nebenwirkungen, z. B. motorisch-kognitive Beeinträchtigungen, machen sie besonders für ältere Patienten zum Risiko. Über das Ausmaß dieses Problems in Deutschland und die Sichtweise der Patienten ist wenig bekannt.

Fragestellung. Untersucht werden sollte, wie viele Patienten Schlaf- und Beruhigungsmittel im Krankenhaus einnehmen, welche Wirkungen und Nebenwirkungen sie erleben und ob die Einnahme dieser Medikamente zum Wunsch nach Weiterverordnung führt.

Methode. Im Rahmen einer Querschnittsstudie sollten in einem Krankenhaus der Grundund Regelversorgung über einen Zeitraum von sechs Monaten Patienten zur Häufigkeit der Einnahme von Schlaf- und Beruhigungsmitteln und über ihre Erfahrungen mit diesen Medikamenten und den Wunsch nach Weitereinnahme befragt werden. Ihre Aussagen sollten mit den Angaben aus der Patientenakte verglichen werden.

Ergebnisse. Rund $45 \%$ (227/508) der Befragten hatten nach eigener Aussage im Krankenhaus Schlaf- oder Beruhigungsmittel erhalten. Knapp zwei Drittel (148/227) der Patienten kamen während der stationären Behandlung zum ersten Mal in Kontakt mit Schlaf- oder Beruhigungsmitteln. Die Übereinstimmung der Aussagen der Patienten mit der Dokumentation in den Patientenakten war sehr hoch (Cohen's Kappa 0,70). Laut Patientenakten hatten 29,5\% aller interviewten Patienten Medikamente aus der Gruppe der Benzodiazepine oder Z-Substanzen erhalten. Nahezu alle berichteten von Verbesserungen durch die Einnahme; aber auch Nebenwirkungen nannte ein Drittel der Befragten. Über 30 \% (85/227) der Patienten wünschten Schlaf- oder Beruhigungsmittel nach Entlassung aus dem Krankenhaus weitereinzunehmen. Die stärksten Einflussfaktoren für diesen Wunsch waren Vorerfahrungen mit entsprechenden Medikamenten vor dem Krankenhausaufenthalt (adjustierte Odds Ratio (OR): 3,86; 95 \%-Konfidenzintervall (KI): 1,99-7,49) sowie die Wahrnehmung von Verbesserungen durch die Einnahme (OR: 10,23; $95 \%-K I: 2,12-49,28)$.

Schlussfolgerungen. Ältere Patienten erhalten bzw. wünschen im Krankenhaus häufig Schlaf- und Beruhigungsmittel. Diese Erfahrung im Krankenhaus ebnet den Weg für den Wunsch einer Einnahme auch zu Hause. Krankenhausärzte, Pflegepersonal, Hausärzte und Patienten sollten stärker über Alternativen zur pharmakologischen Behandlung und den Risiken von Benzodiazepinen und Z-Substanzen informiert werden - mit dem Ziel einer möglichst effektiven Behandlung von Schlafproblemen und der Vermeidung langfristiger Einnahme. 


\section{Literaturverzeichnis}

Albers S, Klapper D, Konradt U, Walter A, Wolf J: Methodik der empirischen Forschung. Springer-Gabler, Wiesbaden 2007

American Academy of Sleep Medicine: International classification of sleep disorders, revised: diagnostic and coding manual. American Academy of Sleep Medicine, Rochester 2001

American Psychiatric Association: Diagnostic and statistical manual of mental disorders, 4th edition. American Psychiatric Association, Washington DC 1994

Ancoli-Israel S (2005): Sleep and aging: prevalence of disturbed sleep and treatment considerations in older adults. J Clin Psychiatry 66 Suppl 9, 24-30

Ancoli-Israel S (2009): Sleep and its disorders in aging populations. Sleep Med $\underline{10}, 7-11$

Anthierens S, Habraken H, Petrovic M, Deveugele M, De Maeseneer J, Christiaens T (2007): First benzodiazepine prescriptions: qualitative study of patients' perspectives. Can Fam Physician $\underline{53}, 1200-1201$

Authier N, Balayssac D, Sautereau M, Zangarelli A, Courty P, Somogyi AA, Vennat B, Llorca P-M, Eschalier A (2009): Benzodiazepine dependence: Focus on withdrawal syndrome. Ann Pharm Fr $\underline{67}, 408-413$

Ballokova A, Peel NM, Fialova D, Scott IA, Gray LC, Hubbard RE (2014): Use of Benzodiazepines and Association with Falls in Older People Admitted to Hospital: A Prospective Cohort Study. Drugs Aging 31, 299-310

Barker MJ, Greenwood KM, Jackson M, Crowe SF (2004): Persistence of cognitive effects after withdrawal from long-term benzodiazepine use: a meta-analysis. Arch Clin Neuropsychol $\underline{19}, 437-454$

Barter G, Cormack M (1996): The long-term use of benzodiazepines: patients' views, accounts and experiences. Fam Pract $\underline{13}$, 491-497

Bartlett G, Abrahamowicz M, Grad R, Sylvestre M-P, Tamblyn R (2009): Association between risk factors for injurious falls and new benzodiazepine prescribing in elderly persons. BMC Fam Pract $\underline{10}, 1-8$

Baruch Y, Holtom BC (2008): Survey response rate levels and trends in organizational research. Hum Relat $\underline{61}, 1139-1160$ 
Bedell SE, Jabbour S, Goldberg R, Glaser H, Gobble S, Young-Xu Y, Graboys TB, Ravid S (2000): Discrepancies in the use of medications: their extent and predictors in an outpatient practice. Arch Intern Med 160, 2129-2134

Bénard-Laribière A, Noize $P$, Pambrun E, Bazin F, Verdoux H, Tournier M, Bégaud B, Pariente A (2016): Comorbidities and concurrent medications increasing the risk of adverse drug reactions: prevalence in French benzodiazepine users. Eur J Clin Pharmacol 72, 869-876

Billioti de Gage S, Begaud B, Bazin F, Verdoux H, Dartigues J-F, Peres K, Kurth T, Pariente A (2012): Benzodiazepine use and risk of dementia: prospective population based study. BMJ 345, 6231-6231

Billioti de Gage S, Moride Y, Ducruet T, Kurth T, Verdoux H, Tournier M, Pariente A, Begaud B (2014): Benzodiazepine use and risk of Alzheimer's disease: case-control study. BMJ $\underline{349}$, $5205-5205$

Billioti de Gage S, Pariente A, Bégaud B (2015): Is there really a link between benzodiazepine use and the risk of dementia? Expert Opin Drug Saf $\underline{14}, 733-747$

Bogunovic OJ, Greenfield SF (2004): Practical Geriatrics: Use of Benzodiazepines Among Elderly Patients. Psychiatr Serv $\underline{55}, 233-235$

Böhm K, Tesch-Römer C, Ziese T (Hrsg.): Gesundheit und Krankheit im Alter. Robert KochInstitut, Berlin 2009

Breemhaar B, Visser AP, Kleijnen JG (1990): Perceptions and behaviour among elderly hospital patients: Description and explanation of age differences in satisfaction, knowledge, emotions and behaviour. Soc Sci Med $\underline{31}, 1377-1385$

Bruder J, Lucke C, Schramm A, Tews HP, Werner H: Was ist Geriatrie? Expertenkommission der Deutschen Gesellschaft für Geriatrie und Deutschen Gesellschaft für Gerontologie zur Definition des Faches Geriatrie. o. Verl., Rügheim 1991

Chaudhuri A, Hey E, Scherer M, Chenot JF (2011): Was wissen Patienten über ihre Medikamente? (45. Kongress für Allgemeinmedizin und Familienmedizin, Forum Medizin 21, Salzburg, 22-24.09.2011). German Medical Science Doc 11/145

Chen L, Bell JS, Visvanathan R, Hilmer SN, Emery T, Robson L, Hughes JM, Tan ECK (2016): The association between benzodiazepine use and sleep quality in residential aged care facilities: a cross-sectional study. BMC Geriatr $\underline{16}, 196$

Cook JM, Biyanova T, Masci C, Coyne JC (2007): Older Patient Perspectives on Long-Term Anxiolytic Benzodiazepine Use and Discontinuation: A Qualitative Study. J Gen Intern Med $\underline{22}, 1094-1100$ 
Cook PJ, Huggett A, Graham-Pole R, Savage IT, James IM (1983): Hypnotic accumulation and hangover in elderly inpatients: a controlled double-blind study of temazepam and nitrazepam. Br Med J Clin Res Ed 286, 100-102

Cook PJ, Flanagan R, James IM (1984): Diazepam tolerance: effect of age, regular sedation, and alcohol. Br Med J Clin Res Ed 289, 351-353

Cusack BJ (2004): Pharmacokinetics in older persons. Am J Geriatr Pharmacother 2, 274302

Deutsche Hauptstelle für Suchtfragen e.V.: DHS Jahrbuch Sucht 2017. Pabst, Lengerich 2017

DGSM (2009): S3-Leitlinie Nicht erholsamer Schlaf/Schlafstörungen. Somnologie 13, 4-160

DGSM (2017): S3-Leitlinie Nicht erholsamer Schlaf/Schlafstörungen. Kapitel „Insomnien bei Erwachsenen“ (AWMF-Registernummer 063-003), Update 2016. Somnologie 21, 2-44

Diem SJ, Ewing SK, Stone KL, Ancoli-Israel S, Redline S, Ensrud KE (2014): Use of nonbenzodiazepine sedative hypnotics and risk of falls in older men. J Gerontol Geriatr Res $\underline{3}$, 158

Dolder C, Nelson M, McKinsey J (2007): Use of non-benzodiazepine hypnotics in the elderly: are all agents the same? CNS Drugs $\underline{21}, 389-405$

Dündar Y, Dodd S, Strobl J, Boland A, Dickson R, Walley T (2004): Comparative efficacy of newer hypnotic drugs for the short-term management of insomnia: a systematic review and meta-analysis. Hum Psychopharmacol $\underline{19}$, 305-322

Edwards C, Bushnell J, Ashton C, Rawlins M (1991): Hospital prescribing and usage of hypnotics and anxiolytics. Br J Clin Pharmacol 31, 190-192

Estler C-J, Schmidt H: Pharmakologie und Toxikologie: für Studium und Praxis; mit 281 Tabellen. Schattauer, Stuttgart 2007

European Medicines Agency: Guideline on medicinal products for the treatment of insomnia. European Medicines Agency, London 2011

Fairweather DB, Kerr JS, Hindmarch I (1992): The effects of acute and repeated doses of zolpidem on subjective sleep, psychomotor performance and cognitive function in elderly volunteers. Eur J Clin Pharmacol $\underline{43}, 597-601$

Finkle WD, Der JS, Greenland S, Adams JL, Ridgeway G, Blaschke T, Wang Z, Dell RM, VanRiper KB (2011): Risk of fractures requiring hospitalization after an initial prescription for Zolpidem, Alprazolam, Lorazepam, or Diazepam in older adults. J Am Geriatr Soc $\underline{59}$, 18831890 
Fleiss JL, Cohen J (1973): The equivalence of weighted Kappa and the intraclass correlation coefficient as measures of reliability. Educ Psychol Meas $\underline{33}$, 613-619

Foy A, Drinkwater V, March S, Mearrick P (1986): Confusion after admission to hospital in elderly patients using benzodiazepines. Br Med J Clin Res Ed 293, 1072

Gaillot J, Heusse D, Hougton GW, Marc Aurele J, Dreyfus JF (1983): Pharmacokinetics and metabolism of Zopiclone. Pharmacology 27, 76-91

Giron MST, Forsell Y, Bernsten C, Thorslund M, Winblad B, Fastbom J (2001): Psychotropic drug use in elderly people with and without dementia. Int J Geriatr Psychiatry 16, 900-906

Glaeske G, Schicktanz C, Tholen K, Windt R, Koller D, Kretschmer C, Stahn D, Tamminga M BARMER GEK Arzneimittelreport. Asgard, Siegburg 2013

Glass J, Lanctôt KL, Herrmann N, Sproule BA, Busto UE (2005): Sedative hypnotics in older people with insomnia: meta-analysis of risks and benefits. BMJ $\underline{331}, 1169$

Gleason PP, Schulz R, Smith NL, Newsom JT, Kroboth PD, Kroboth FJ, Psaty BM (1998): Correlates and prevalence of benzodiazepine use in community-dwelling elderly. J Gen Intern Med $\underline{13}, 243-250$

Gray SL, Dublin S, Yu O, Walker R, Anderson M, Hubbard RA, Crane PK, Larson EB (2016): Benzodiazepine use and risk of incident dementia or cognitive decline: prospective population based study. BMJ $\underline{352}$, i90

Gunja N (2013): In the Zzz Zone: The effects of Z-Drugs on human performance and driving. J Med Toxicol $\underline{9}, 163-171$

Hajak G (2001): Epidemiology of severe insomnia and its consequences in Germany. Eur Arch Psychiatry Clin Neurosci 251, 49-56

Hajak G, Müller WE, Wittchen H-U, Pittrow D, Kirch W (2003): Abuse and dependence potential for the non-benzodiazepine hypnotics zolpidem and zopiclone: a review of case reports and epidemiological data. Addiction $\underline{98}, 1371$

Halfens R, Lendfers M, Cox K (1991): Sleep medication in Dutch hospitals. J Adv Nurs $\underline{16}$, 1422-1427

Hallstrom C, Lader M (1981): Benzodiazepine withdrawal phenomena. Int Pharmacopsychiatry $\underline{16}, 235-244$

Heinemann S, Himmel W (2017): Searching for factors that may reduce the use of benzodiazepines in hositals -a survey of hospital doctors and nurses. Int J Clin Pharmacol Ther $\underline{55}$, $1-6$ 
Heinemann S, Weiß V, Straube K, Nau R, Grimmsmann T, Himmel W, Hummers-Pradier E (2016b): Understanding and reducing the prescription of hypnotics and sedatives at the interface of hospital care and general practice: a protocol for a mixed-methods study. BMJ Open $\underline{6}, 1-6$

Hoffmann F (2013): Benefits and risks of benzodiazepines and Z-drugs: comparison of perceptions of GPs and community pharmacists in Germany. GMS Ger Med Sci 11, 1-7

Hoffmann F, Glaeske G (2014): Benzodiazepinhypnotika, Zolpidem und Zopiclon auf Privatrezept. Nervenarzt $\underline{85}, 1402-1409$

Hollister LE, Motzenbecker FP, Degan RO (1961): Withdrawal reactions from chlordiazepoxide (“Librium"). Psychopharmacologia 2, 63-68

Holt S, Schmiedl S, Thürmann PA (2010): Potentially inappropriate medications in the elderly: the PRISCUS list. Dtsch Ärztebl Int 107, 543-551

Johnson CF, Frei C, Downes N, McTaggart SA, Akram G (2016): Benzodiazepine and zhypnotic prescribing for older people in primary care: a cross-sectional population-based study. Br J Gen Pr $\underline{66}, 410-415$

Johnson EO, Roehrs T, Roth T, Breslau N (1998): Epidemiology of alcohol and medication as aids to sleep in early adulthood. Sleep $\underline{21}, 178-186$

Kerzman H, Baron-Epel O, Toren O (2005): What do discharged patients know about their medication? Patient Educ Couns $\underline{56}, 276-282$

King MB, Gabe J, Williams P, Rodrigo EK (1990): Long term use of benzodiazepines: the views of patients. Br J Gen Pract $\underline{40}, 194-196$

Lader M (1991): History of benzodiazepine dependence. J Subst Abuse Treat $\underline{8}, 53-59$

Lagnaoui R, Moore N, Longy-Boursier M, Baumevieille M, Bégaud B (2001): Benzodiazepine use in patients hospitalized in a department of internal medicine: frequency and clinical correlates. Pharmacoepidemiol Drug Saf $\underline{10}, 531-535$

Lagnaoui R, Bégaud B, Moore N, Chaslerie A, Fourrier A, Letenneur L, Dartigues J-F, Moride $Y$ (2002): Benzodiazepine use and risk of dementia: A nested case-control study. $J$ Clin Epidemiol 55, 314-318

Landis JR, Koch GG (1977): The measurement of observer agreement for categorical data. Biometrics $\underline{33}, 159-174$

Leufkens TRM, Lund JS, Vermeeren A (2009): Highway driving performance and cognitive functioning the morning after bedtime and middle-of-the-night use of gaboxadol, zopiclone and zolpidem. J Sleep Res $\underline{18}$, 387-396 
Lowe CJ, Raynor DK, Courtney EA, Purvis J, Teale C (1995): Effects of self medication programme on knowledge of drugs and compliance with treatment in elderly patients. BMJ $\underline{310}$, 1229-1231

Lund HG, Reider BD, Whiting AB, Prichard JR (2010): Sleep patterns and predictors of disturbed sleep in a large population of college students. J Adolesc Health $\underline{46}, 124-132$

Makaryus AN, Friedman EA (2005): Patients' understanding of their treatment plans and diagnosis at discharge. Mayo Clin Proc $\underline{80}, 991-994$

Maust DT, Kales HC, Wiechers IR, Blow FC, Olfson M (2016): No end in sight: benzodiazepine use in older adults in the united states. J Am Geriatr Soc $\underline{64}, 2546-2553$

Mets MAJ, de Vries JM, de Senerpont Domis LM, Volkerts ER, Olivier B, Verster JC (2011): Next-day effects of Ramelteon (8 mg), Zopiclone $(7.5 \mathrm{mg})$, and placebo on highway driving performance, memory functioning, psychomotor performance, and mood in healthy adult subjects. Sleep $\underline{34}, 1327-1334$

Miller LG, Greenblatt DJ, Barnhill JG, Shader RI (1988): Chronic benzodiazepine administration. I. Tolerance is associated with benzodiazepine receptor downregulation and decreased gamma-aminobutyric acidA receptor function. J Pharmacol Exp Ther 246, 170-176

Mura T, Proust-Lima C, Akbaraly T, Amieva H, Tzourio C, Chevassus H, Picot M-C, Jacqumin-Gadda H, Berr C (2013): Chronic use of benzodiazepines and latent cognitive decline in the elderly: Results from the Three-city study. Eur Neuropsychopharmacol $\underline{23}, 212-$ 223

Nakao M, Sato M, Nomura K, Yano E (2009): Benzodiazepine prescription and length of hospital stay at a Japanese university hospital. Biopsychosoc Med $\underline{3}, 10$

NICE-National Institute of Clinical Excellence: Guidance on the use of zaleplon, zolpidem and zopiclone for the short-term management of insomnia. NICE guidance, London 2004

Noble T, Spiroulias M, White JM (1993): Determinants of benzodiazepine prescribing and administration in a public hospital. Pharmacopsychiatry $\underline{26}, 11-14$

O’Brien CP (2005): Benzodiazepine use, abuse, and dependence. J Clin Psychiatry $\underline{66}$, 2833

Ohayon MM (2002): Epidemiology of insomnia: what we know and what we still need to learn. Sleep Med Rev $\underline{6}, 97-111$

O'Reilly R, Rusnak C (1990): The use of sedative-hypnotic drugs in a university teaching hospital. Can Med Assoc J 142, 585-589 
Petitjean S, Ladewig D, Meier CR, Amrein R, Wiesbeck GA (2007): Benzodiazepine prescribing to the Swiss adult population: results from a national survey of community pharmacies. Int Clin Psychopharmacol 22, 292-298

Petursson H, Lader MH (1981): Benzodiazepine dependence. Br J Addict 트, 133-145

Pinto Jr. LR, Alves RC, Caixeta E, Fontenelle JA, Bacellar A, Poyares D, Aloe F, Rizzo G, Minhoto G, Bittencourt LR, et al. (2010): New guidelines for diagnosis and treatment of insomnia. Arq Neuropsiquiatr $\underline{68}, 666-675$

Pullar T, Roach P, Mellor EJ, McNeece J, Judd A, Feely M, Cooke J (1989): Patients‘ knowledge concerning their medications on discharge from hospital. J Clin Pharm Ther $\underline{14}$, $57-59$

Puustinen J, Nurminen J, Kukola M, Vahlberg T, Laine K, Kivelä S-L (2007): Associations between use of benzodiazepines or related drugs and health, physical abilities and cognitive function. Drugs Aging 24, 1045-1059

Ray WA, Griffin MR, Downey W (1989): Benzodiazepines of long and short elimination halflife and the risk of hip fracture. JAMA 262, 3303-3307

Riss J, Cloyd J, Gates J, Collins S (2008): Benzodiazepines in epilepsy: pharmacology and pharmacokinetics. Acta Neurol Scand $\underline{118}, 69-86$

Robert Koch-Institut (Hrsg.): Themenheft 27: Schlafstörungen. Gesundheitsberichterstattung des Bundes; Robert Koch-Institut, Berlin 2005

Rosenbaum JF (2005): Attitudes toward benzodiazepines over the years. J Clin Psychiatry 66 Suppl 2, 4-8

Rote Liste: ROTE LISTE 2017 Arzneimittelverzeichnis für Deutschland. Rote Liste Service, Frankfurt/Main 2017

Schlack R, Hapke U, Maske U, Busch M, Cohrs S (2013): Häufigkeit und Verteilung von Schlafproblemen und Insomnie in der deutschen Erwachsenenbevölkerung. Bundesgesundheitsblatt - Gesundheitsforschung - Gesundheitsschutz 트, 740-748

Scholl A: Die Befragung. UVK, Konstanz 2015

Schutte-Rodin S, Broch L, Buysse D, Dorsey C, Sateia M (2008): Clinical Guideline for the Evaluation and Management of Chronic Insomnia in Adults. J Clin Sleep Med 4, 487-504

Schwabe U, Paffrath D (Hrsg.): Arzneiverordnungs-Report 2015. Springer, Berlin, Heidelberg 2015 
Schwabe U, Paffrath D (Hrsg.): Arzneiverordnungs-Report 2017. Springer, Berlin, Heidelberg 2017

Sirdifield C, Chipchase SY, Owen S, Siriwardena AN (2017): A Systematic Review and Meta-Synthesis of Patients' Experiences and Perceptions of Seeking and Using Benzodiazepines and Z-Drugs: Towards Safer Prescribing. Patient 10, 1-15

Siriwardena AN, Qureshi Z, Gibson S, Collier S, Latham M (2006): GPs' attitudes to benzodiazepine and 'Z-drug' prescribing: a barrier to implementation of evidence and guidance on hypnotics. Br J Gen Pr $\underline{56}, 964-967$

Siriwardena AN, Qureshi MZ, Dyas JV, Middleton H, Orner R (2008): Magic bullets for insomnia? Patients' use and experiences of newer ( $Z$ drugs) versus older (benzodiazepine) hypnotics for sleep problems in primary care. Br J Gen Pract $\underline{58}, 417-422$

Siriwardena AN, Huedo-Medina TB, Middlemass J, Klonzakis M, Kirsch I: How effective are Z-drug hypnotics for treatment of adult insomnia? Meta-analysis of data submitted to the Food and Drug Administration; Scottish Exhibition and Conference Centre, Glasgow, UK 2012

Sjahid SI, Van Der Linden PD, Stricker BHC (1998): Agreement between the pharmacy medication history and patient interview for cardiovascular drugs: the Rotterdam elderly study. $\mathrm{Br}$ J Clin Pharmacol $\underline{45}$, 591-595

Somers A, Robays H, Audenaert K, Maele GV, Bogaert M, Petrovic M (2011): The use of hypnosedative drugs in a university hospital: has anything changed in 10 years? Eur $\mathrm{J}$ Clin Pharmacol $\underline{67}, 723-729$

Sonnenberg CM, Bierman EJM, Deeg DJH, Comijs HC, van Tilburg W, Beekman ATF (2012): Ten-year trends in benzodiazepine use in the Dutch population. Soc Psychiatry Psychiatr Epidemiol 47, 293-301

Stasio MJ, Curry K, Sutton-Skinner KM, Destinee M. G (2008): Over-the-counter medication and herbal or dietary supplement use in college: dose frequency and relationship to selfreported distress. J Am Coll Health $\underline{56}, 535-548$

Statistisches Bundesamt: Gesundheit - Diagnosedaten der Patienten und Patientinnen in Krankenhäusern (einschl. Sterbe- und Stundenfälle) 2014. (Fachserie 12 Reihe 6.2.1); Statistisches Bundesamt, Wiesbaden 2015

Sternbach LH (1971): 1,4-Benzodiazepine. Chemie und Betrachtungen zur Beziehung zwischen Struktur und Wirkung. Angew Chem $\underline{83}, 70-79$

Sternbach LH (1979): The benzodiazepine story. J Med Chem 22, 1-7 
Tulner LR, Kuper IMJA, Frankfort SV, van Campen JPCM, Koks CHW, Brandjes DPM, Beijnen JH (2009): Discrepancies in reported drug use in geriatric outpatients: Relevance to adverse events and drug-drug interactions. Am J Geriatr Pharmacother $\underline{7}$, 93-104

Vermeeren A, Danjou PE, O'Hanlon JF (1998): Residual effects of evening and middle-ofthe-night administration of zaleplon 10 and $20 \mathrm{mg}$ on memory and actual driving performance. Hum Psychopharmacol Clin Exp $\underline{13}$, S98-S107

Vermeeren A, Vuurman EFPM, Leufkens TRM, Van Leeuwen CJ, Van Oers ACM, Laska E, Rico S, Steinberg F, Roth T (2014): Residual effects of low-dose sublingual Zolpidem on highway driving performance the morning after middle-of-the-night use. Sleep $\underline{37}$, 489-496

Wagner AK, Zhang F, Soumerai SB, Walker AM, Gurwitz JH, Glynn RJ, Ross-Degnan D (2004): Benzodiazepine use and hip fractures in the elderly: who is at greatest risk? Arch Intern Med 164, 1567-1572

Wang PS, Bohn RL, Glynn RJ, Mogun H, Avorn J (2001a): Hazardous benzodiazepine regimens in the elderly: effects of half-life, dosage, and duration on risk of hip fracture. Am $J$ Psychiatry $\underline{158}, 892-898$

Wang PS, Bohn RL, Glynn RJ, Mogun H, Avorn J (2001b): Zolpidem use and hip fractures in older people. J Am Geriatr Soc 49, 1685-1690

Warie H, Petrovic M, Somers A, Mariman A, Robays H, Pevernagie D (2003): The use of hypnosedative drugs in a university hospital setting. Acta Clin Belg $\underline{58}, 225-232$

Woodward M (1999): Hypnosedatives in elderly: guide to appropriate use. CNS Drugs 11, 263-279 [https://doi.org/10.2165/00023210-199911040-00003; Zugriff am 05.08.2015]

Wright BT, Gluszek CF, Heldt SA (2014): The effects of repeated zolpidem treatment on tolerance, withdrawal-like symptoms, and GABAA receptor mRNAs profile expression in mice: Comparison with diazepam. Psychopharmacology (Berl) 231, 2967-2979

Yu N-W, Chen P-J, Tsai H-J, Huang C-W, Chiu Y-W, Tsay W-I, Hsu J, Chang C-M (2017): Association of benzodiazepine and Z-drug use with the risk of hospitalisation for fall-related injuries among older people: a nationwide nested case-control study in Taiwan. BMC Geriatr $\underline{17}$

Zisselman MH, Rovner BW, Kelly KG, Woods C (1994): Benzodiazepine utilization in a university hospital. Am J Med Qual $\underline{9}, 138-141$ 
10. Anhang

\subsection{Fragebogen inklusive Grundauszählung}

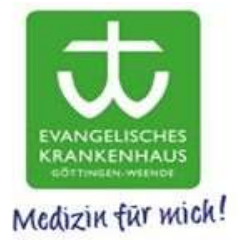

Fachabteilung:

Sehr geehrte Patientin, sehr geehrter Patient,

in diesem Fragebogen geht es um Ihre Erfahrungen mit Beruhigungs- und Schlafmitteln, also mit Medikamenten, die man manchmal bei Angst, Unruhe oder Schlaflosigkeit z. B. in der ungewohnten Umgebung des Krankenhauses verordnet erhält.

Auch wenn Sie jetzt keine Beruhigungs- und Schlafmittel einnehmen, ist es für unsere Studie sehr wichtig, dass Sie mit uns gemeinsam diesen Fragebogen ausfüllen.

Befragung zur Einnahme von Beruhigungs- und Schlafmitteln während des stationären Aufenthaltes

Haben Sie im Rahmen Ihres Aufenthalts (hier im Krankenhaus) Schlaf- oder Beruhigungsmittel verordnet bekommen?

\begin{tabular}{|l|l|l|}
\hline ja & nein & weiß nicht \\
& (bitte weiter zu Frage 8) & (bitte weiter zu Frage 8) \\
$227(44,7 \%)$ & $266(52,4 \%)$ & $15(2,9 \%)$
\end{tabular}


Wie viele Medikamente haben Sie erhalten?

Können Sie mir den Namen des oder der Schlaf- oder Beruhigungsmittel nennen?

Anzahl der Medikamente

$\begin{array}{ll}1 & 196(87,1 \%) \\ 2 & 27(12 \%) \\ 3 & 2(0,9 \%)\end{array}$

Ein Viertel aller Patienten (58/227), die ein Schlaf- oder Beruhigungsmittel laut eigener Aussage erhalten hatten, konnten den Namen des Medikaments nennen.

$\begin{aligned} & \text { Wie oft haben Sie dieses Medikamente/das Medikament während ihres Krankenhausaufent- } \\
& \text { haltes eingenommen? }\end{aligned}$
\begin{tabular}{l|l|l} 
einmal & mehrmals mit Unterbrechung \\
$\mathbf{3 1}(13,7 \%)$ & $\mathbf{8 0}(35,2 \%)$ & täglich \\
& & $116(51,1 \%)$ \\
\hline
\end{tabular}

\begin{tabular}{|c|c|c|c|}
\hline & ja & nein & weiß ich nicht \\
\hline Einschlafstörungen & $166(73,1 \%)$ & $53(23,3 \%)$ & $8(3,5 \%)$ \\
\hline Durchschlafstörungen & $134(59,0 \%)$ & $85(37,4 \%)$ & $8(3,5 \%)$ \\
\hline Schmerzen & $46(20,4 \%)$ & $164(72,6 \%)$ & $16(7,1 \%)$ \\
\hline Nervosität & $84(37,2 \%)$ & $128(56,6 \%)$ & $14(6,2 \%)$ \\
\hline Angstzustände & $49(21,6 \%)$ & $170(74,9 \%)$ & $8(3,5 \%)$ \\
\hline $\begin{array}{l}\text { Bessere Toleranz der Umgebungszu- } \\
\text { stände }\end{array}$ & $104(45,8 \%)$ & $107(47,1 \%)$ & $16(7,1 \%)$ \\
\hline
\end{tabular}


Haben Sie folgende Nebenwirkungen durch die Einnahme dieses/dieser Beruhigungs- oder Schlafmitteln wahrgenommen?

\begin{tabular}{|l|l|l|}
\hline & ja & nein \\
\hline Kopfschmerzen & $\mathbf{5}(2,2 \%)$ & $\mathbf{2 2 2}(97,8 \%)$ \\
\hline Schwindel & $\mathbf{2 2}(9,7 \%)$ & $\mathbf{2 0 5}(90,3 \%)$ \\
\hline Übelkeit & $\mathbf{1 7}(7,5 \%)$ & $\mathbf{2 1 0}(95,5 \%)$ \\
\hline Benommenheit & $\mathbf{2 5}(11,0 \%)$ & $\mathbf{2 0 2}(89,0 \%)$ \\
\hline Verwirrtheit & $\mathbf{5 ( 2 , 2 \% )}$ & $\mathbf{2 2 2}(97,8 \%)$ \\
\hline Konzentrationsstörungen & $\mathbf{1 4}(6,2 \%)$ & $\mathbf{2 1 3}(93,8 \%)$ \\
\hline Stürze & $\mathbf{3 ( 1 , 3 \% )}$ & $\mathbf{2 2 4}(98,7 \%)$ \\
\hline Gangunsicherheit & $\mathbf{1 3}(5,7 \%)$ & $\mathbf{2 1 4}(94,3 \%)$ \\
\hline Knochenbrüche & 0 & $\mathbf{2 2 7}$ \\
\hline Sehstörungen & $\mathbf{1 1}(4,9 \%)$ & $\mathbf{2 1 6}(95,1 \%)$ \\
\hline Zittern & $\mathbf{4}(1,8 \%)$ & $\mathbf{2 2 3}(98,25)$ \\
\hline Schläfrigkeit & $\mathbf{4 6}(20,3 \%)$ & $\mathbf{1 8 1}(79,7 \%)$ \\
\hline Vergesslichkeit & $\mathbf{7 ( 3 , 1 \% )}$ & $\mathbf{2 1 9}(96,9 \%)$ \\
\hline & & \\
\hline
\end{tabular}

Andere:

Nachtschweiß, Aufschrecken, Unruhe, das Gefühl „flatterig“ sein, Frieren, Alpträume (zweimal genannt), Desorientiertheit nach dem Aufwachen (zweimal genannt), Artikulationsschwierigkeiten, Diarrhoe 
Würden Sie das oder die Schlaf- oder Beruhigungsmittel auch nach der Entlassung aus dem Krankenhaus gern weiternehmen?

ja $\quad$ nein

$85(37,4 \%)$ $142(62,6 \%)$

Gerne können Sie uns zu dieser Frage auch noch etwas ausführlicher antworten, z.B. warum Sie gerne dieses Medikament weiter einnehmen möchten:

Wie beurteilen Sie insgesamt die Wirkung dieses/dieser Schlaf- und Beruhigungsmittel(s)? eher positiv eher negativ

$192(85,7 \%)$ $32(14,3 \%)$

Einnahme von Beruhigungs- und Schlafmitteln vor dem stationären Aufenthalt

Haben Sie vor Ihrem Krankenhausaufenthalt Schlaf- oder Beruhigungsmittel eingenommen?

\begin{tabular}{|l|l|l|}
\hline ja & $\begin{array}{l}\text { nein } \\
\text { (bitte weiter zu Frage 17) }\end{array}$ & $\begin{array}{l}\text { weiß ich nicht } \\
\text { (bitte weiter zu Frage 17) }\end{array}$ \\
\hline $\mathbf{8 9}(17,5 \%)$ & $\mathbf{4 1 2 ( 8 1 , 1 \% )}$ & $\mathbf{7 ( 1 , 4 \% )}$ \\
\hline
\end{tabular}

Wie viele Medikamente haben Sie eingenommen?

Anzahl der Medikamente 


\begin{tabular}{|l|l|}
\hline $\begin{array}{l}\text { Haben Sie dieses Schlaf- oder Beruhigungsmittel auch noch in den letzten } 6 \text { Monaten ein- } \\
\text { genommen? }\end{array}$ \\
\hline ja & nein \\
\hline $\mathbf{8 0}(89,9 \%)$ & $\mathbf{9 ( 1 0 , 1 \% )}$ \\
\hline
\end{tabular}

Über welchen Zeitraum haben Sie dieses Schlaf- und Beruhigungsmittel? Eingenommen?

\begin{tabular}{|l|l|l|}
\hline bis zu 4 Wochen & bis zu 1 Jahr & über 1 Jahr \\
\hline $\mathbf{1 1}(12,2 \%)$ & $\mathbf{1 5}(20,0 \%)$ & $\mathbf{6 0}(67,8 \%)$ \\
\hline
\end{tabular}

Was hat sich für Sie durch die Einnahme des oder der Schlaf- oder Beruhigungsmittel verbessert?

\begin{tabular}{|l|l|l|l|}
\hline & ja & nein & weiß ich nicht \\
\hline Einschlafstörungen & $\mathbf{8 1 ( 1 0 0 \% )}$ & 0 & 0 \\
\hline Durchschlafstörungen & $\mathbf{6 1}(96,8 \%)$ & 0 & $\mathbf{2 ( 3 , 2 \% )}$ \\
\hline Schmerzen & $\mathbf{1 8 ( 2 0 , 2 \% )}$ & $\mathbf{7 1}(79,8 \%)$ & 0 \\
\hline Nervosität & $\mathbf{4 0 ( 4 4 , 9 \% )}$ & $\mathbf{4 5 ( 5 0 , 6 \% )}$ & $\mathbf{4 ( 4 , 5 \% )}$ \\
\hline Angstzustände & $\mathbf{2 7 ( 3 0 , 3 \% )}$ & $\mathbf{6 0 ( 6 7 , 4 \% )}$ & $\mathbf{2 ( 2 , 3 \% )}$ \\
\hline
\end{tabular}




\begin{tabular}{|c|c|c|}
\hline \multicolumn{3}{|c|}{$\begin{array}{l}\text { Haben Sie folgende Nebenwirkungen durch die Einnahme von Beruhigungs- oder Schla } \\
\text { mitteln wahrgenommen? }\end{array}$} \\
\hline & ja & nein \\
\hline Kopfschmerzen & $4(4,5 \%)$ & $85(95,5 \%)$ \\
\hline Schwindel & $8(9,0 \%)$ & $81(91,0 \%)$ \\
\hline Übelkeit & $8(9,0 \%)$ & $81(91,0 \%)$ \\
\hline Benommenheit & $12(13,5 \%)$ & $77(86,5 \%)$ \\
\hline Verwirrtheit & $1(1,1 \%)$ & $87(98,9 \%)$ \\
\hline Konzentrationsstörungen & $9(10,1 \%)$ & $80(89,9 \%)$ \\
\hline Stürze & $8(9,0 \%)$ & $81(91,0 \%)$ \\
\hline Gangunsicherheit & $6(6,7 \%)$ & $83(93,3 \%)$ \\
\hline Knochenbrüche & $\mathbf{1}(1,1 \%)$ & $88(96,6 \%)$ \\
\hline Sehstörungen & $3(3,4 \%)$ & $86(95,1 \%)$ \\
\hline Zittern & 0 & 85 \\
\hline Schläfrigkeit & $21(23,6 \%)$ & $68(76,4 \%)$ \\
\hline Vergesslichkeit & $6(7,1 \%)$ & $79(92,9 \%)$ \\
\hline Andere & & \\
\hline
\end{tabular}

\begin{tabular}{|l|l|}
\hline $\begin{array}{l}\text { Würden Sie die Schlaf- oder Beruhigungsmittel auch nach der Entlassung aus dem Kran- } \\
\text { kenhaus gerne weiterhin einnehmen? }\end{array}$ \\
\hline ja & nein \\
\hline $\mathbf{7 1}(79,8 \%)$ & $\mathbf{1 8}(20,2 \%)$ \\
\hline
\end{tabular}

Gerne können Sie uns zu dieser Frage auch noch etwas ausführlicher antworten, z.B. warum Sie gerne dieses oder diese Schlaf- oder Beruhigungsmittel weiter einnehmen möchten: 
15. Haben Sie versucht das oder die Schlaf- oder Beruhigungsmittel abzusetzen?

\begin{tabular}{|l|l|}
\hline ja & nein \\
\hline $43(48,3 \%)$ & $46(51,7 \%)$ \\
\hline
\end{tabular}

Gerne können Sie uns zu dieser Frage auch noch etwas ausführlicher antworten, z.B. auf welche Beruhigungs- und Schlafmittel Sie verzichten möchten und warum:

16. Wie beurteilen Sie insgesamt die Wirkung dieses Schlaf- und Beruhigungsmittels?

\begin{tabular}{|l|l|}
\hline eher positiv & eher negativ \\
\hline $\mathbf{7 8}(87,6 \%)$ & $\mathbf{1 1}(12,4 \%)$ \\
\hline
\end{tabular}

Auch wenn alle Fragen für Sie nicht zutreffend sind, möchten wir Sie bitten, Frage 17 und 18 zu beantworten:

Wir bitten Sie noch um zwei persönliche Angaben zu Ihrer Person

17. Ihr Geschlecht

Weiblich

Männlich

Ihr Alter:

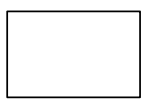

Vielen Dank für Ihre Mitarbeit! 
$\underline{\text { Zweiter Teil des Fragebogens }}$

Verordnungsdaten

\begin{tabular}{|c|c|c|c|c|}
\hline Substanz & nie & einmalig & mehrmals mit Unterbrechung & täglich \\
\hline $\begin{array}{l}\text { Langwirksame Benzodiaze- } \\
\text { pine }\end{array}$ & & & & \\
\hline Diazepam & & & & \\
\hline Flurazepam & & & & \\
\hline Bromazepam & & & & \\
\hline Nitrazepam & & & & \\
\hline Flunitrazepam & & & & \\
\hline $\begin{array}{l}\text { Kurz- und mittellang wirk- } \\
\text { same Benzodiazepine }\end{array}$ & & & & \\
\hline Temazepam & & & & \\
\hline Lorazepam & & & & \\
\hline Oxazepam & & & & \\
\hline Lormetazepam & & & & \\
\hline Z-Substanzen & & & & \\
\hline Zolpidem & & & & \\
\hline Zopiclon & & & & \\
\hline $\begin{array}{l}\text { Andere Benzodiazepine o- } \\
\text { der Z-Substanzen } \\
\text { bitte nennen: } \\
\text { FREITEXT }\end{array}$ & & & & \\
\hline $\begin{array}{l}\text { Andere Sedativa (PRISCUS- } \\
\text { Liste) }\end{array}$ & & & & \\
\hline Doxylamin & & & & \\
\hline Diphenhydramin & & & & \\
\hline Chloralhydrat & & & & \\
\hline
\end{tabular}




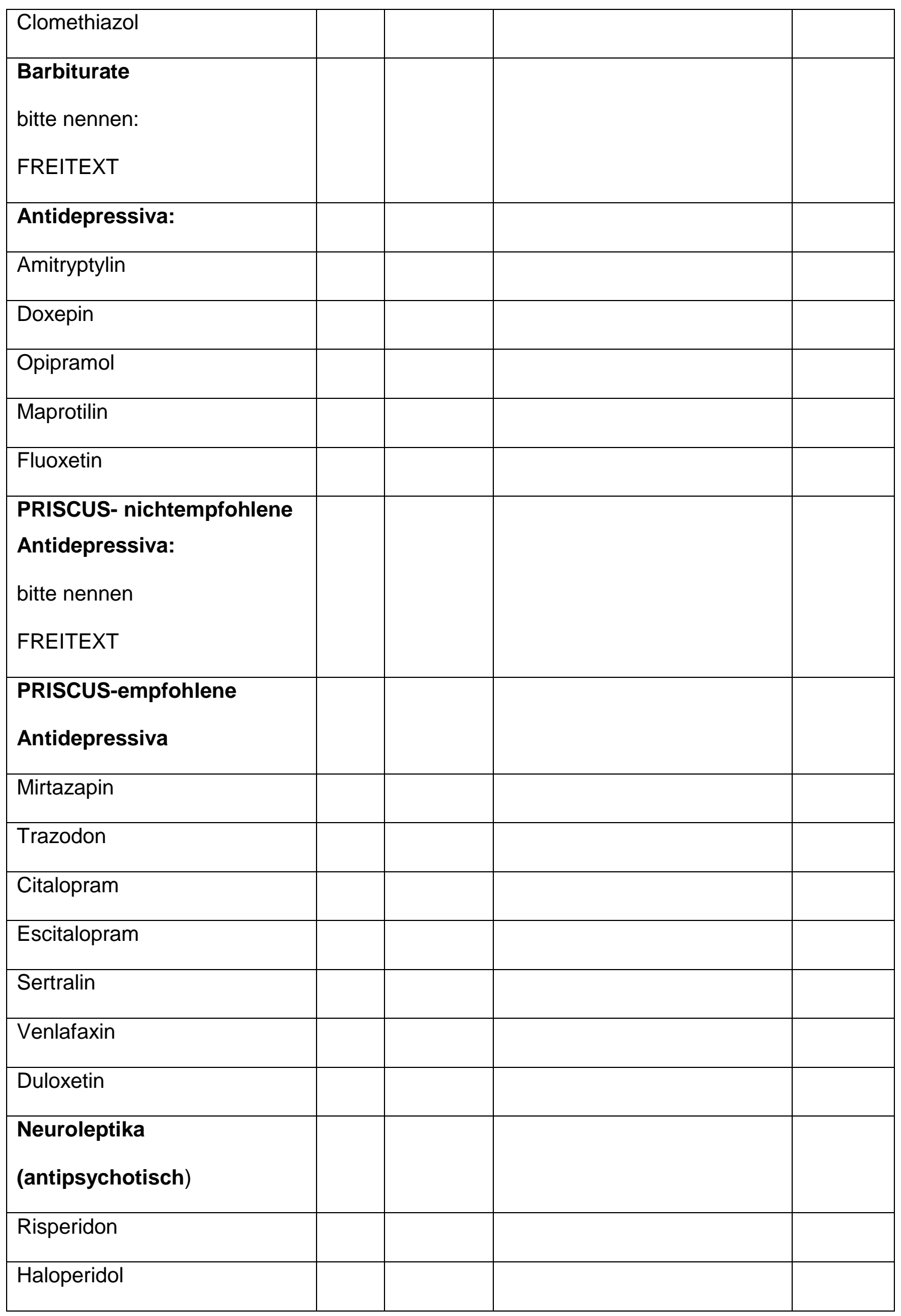




\begin{tabular}{|l|l|l|l|l|}
\hline Quetiapin & & & & \\
\hline Olanzapin & & & & \\
\hline Clozapin & & & & \\
\hline Neuroleptika (sedierend) & & & & \\
\hline Melperon & & & & \\
\hline Pipamperon & & & & \\
\hline Prothipendyl & & & & \\
\hline Andere Neuroleptika: & & & & \\
\hline FREITEXT & & & & \\
\hline
\end{tabular}

Prof. Dr. Roland Nau

Chefarzt des Geriatrischen Zentrums

Ev. Krankenhaus-Weende

An der Lutter 24, 37075 Göttingen

Tel.:0551-5034-1560

rnau@ekweende.de
Prof. Dr. Eva Hummers-Pradier

Direktorin des Instituts für Allgemeinmedizin

Universitätsmedizin Göttingen

Humboldtallee 38, 37073 Göttingen

Tel.: 0551-39-22638

iprimas@gwdg.de 
10.2 Informationen über die Studie für Patienten und Informed Consent

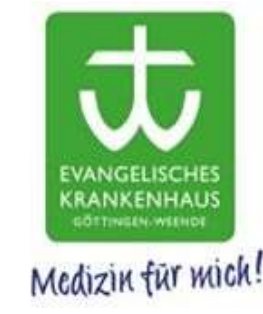

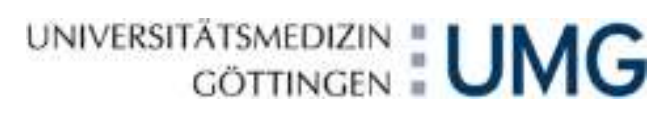

\section{Bitte um Teilnahme an einer wissenschaftlichen Studie Beruhigungs- und Schlafmittel im Krankenhaus}

Sehr geehrte Patientin, sehr geehrter Patient,

das Evangelische Krankenhaus Weende führt in Kooperation mit dem Institut für Allgemeinmedizin der Universitätsmedizin Göttingen eine Untersuchung zur Einnahme von Beruhigungs- und Schlafmitteln im Krankenhaus durch. Wir - Prof. Eva Hummers-Pradier und Prof. Roland Nau - möchten Sie bitten, an dieser Befragung teilzunehmen.

Was ist das Ziel?

Um Angst, Unruhe oder Schlaflosigkeit in der ungewohnten Umgebung eines Krankenhauses zu behandeln, erhalten Patienten manchmal Beruhigungs- oder Schlafmittel. In dieser Untersuchung möchten wir erfahren, ob diese Medikamente auch nach Krankenhausentlassung weiter verordnet werden und ob z. B. Ihr Hausarzt darüber informiert ist. Aber auch über Ihre Erfahrungen mit der Einnahme dieser Medikamente möchten wir mehr erfahren. Wichtig für die Untersuchung ist auch, dass wir alle Innen im Krankenhaus gegebenen Medikamente kennen.

Wie können Sie uns unterstützen?

Wir möchten Sie bitten, die Fragen der Interviewerin/des Interviewers ehrlich zu beantworten. Auch wenn Sie vor der Krankenhausbehandlung oder im Krankenhaus selbst keine Beruhigungs- oder Schlafmittel erhalten haben, bitten wir Sie, uns zumindest zwei Angaben zu Ihrer Person zu nennen.

Weiterhin bitten wir Sie, die Einverständniserklärung zu unterschreiben und der Interviewerin/dem Interviewer zurück zu geben. Nur mit Ihrem Einverständnis dürfen wir den Fragebogen auswerten. 
Mit der Einverständniserklärung erlauben Sie zudem den behandelnden Ärzten im Ev. Krankenhaus Weende, uns Ihre dort erhaltenden Medikamente und die dazugehörenden Diagnosen anonym mitzuteilen.

\section{Wichtig für Sie zu wissen}

Die Dateneingabe und Auswertung findet im Institut für Allgemeinmedizin der Universitätsmedizin Göttingen statt. Ihre persönlichen Daten werden sofort nach Eingang im Institut für Allgemeinmedizin von dem Fragebogen getrennt. Mit Ihrer Einverständniserklärung dürfen die Ärzte im Ev. Krankenhaus Weende uns sagen, welche Medikamente und Diagnosen Sie im Krankenhaus erhalten haben. Dies erfolgt schon nicht mehr mit Ihren Namen, sondern mit einem Nummern-Code. Alle Angaben zu Ihren Medikamenten und Diagnosen lassen sich nur noch durch diesen Nummern-Code Ihnen zuordnen. Das ist eine sog. Pseudonymisierung, die keinen Rückschluss auf Sie als Person mehr zulässt. Dritte erhalten keinen Einblick in Ihre Unterlagen.

Sie können jederzeit die Zustimmung zur Teilnahme an dieser Studie ohne Gründe widerrufen, ohne dass Ihnen hierdurch irgendwelche Nachteile entstehen.

Falls Sie noch Fragen haben, wenden Sie sich bitte an unsere Projektmitarbeiterin im Ev. Krankenhaus Weende, Kati Straube (0551-5034-1761) oder an die Projektmitarbeiterin des Instituts für Allgemeinmedizin, Vivien Weiß (0551-34-14227 oder 22638).

Herzlichen Dank für Ihre Unterstützung!

Prof. Dr. Roland Nau

Chefarzt des Geriatrischen Zentrums

Ev. Krankenhaus-Weende

An der Lutter 24, 37075 Göttingen

Tel. 0551. 5034-1560

rnau@ekweende.de
Prof. Dr. Eva Hummers-Pradier

Direktorin des Instituts für Allgemeinmedizin

Universitätsmedizin Göttingen

Humboldtallee 38, 37073 Göttingen

Tel. 0551. 39-22638

iprimas@gwdg.de 


\subsection{Einverständniserklärung}

\section{UNIVERSITÄTSMEDIZIN $=\mathbf{O}$
GÖTTINGEN $=\mathbf{O}$}

Medizin für mich!

ID

\section{Einverständniserklärung zur Teilnahme an einer wissenschaftlichen Studie Beruhigungs- und Schlafmittel im Krankenhaus}

Ich habe das Informationsblatt der o. g. Umfrage gelesen und verstanden. Ich weiß, dass ich mich bei Fragen jederzeit an Kati Straube oder Frau Weiß wenden kann. Ich weiß, dass die Teilnahme freiwillig und mit keinem unmittelbaren persönlichen Nutzen oder Nachteilen für mich verbunden ist. Ich bin damit einverstanden, dass der beiliegende und von mir ausgefüllte Fragebogen im Institut für Allgemeinmedizin der Universitätsmedizin Göttingen ausgewertet wird. Ich bin damit einverstanden, dass den verantwortlichen Projektmitarbeitern des Instituts für Allgemeinmedizin von meinen behandelnden Ärzten im Ev. Krankenhaus Weende mitgeteilt wird, welche Medikamente (mit Diagnosen) ich vor und während meines Krankenhausaufenthaltes erhalten habe und welche Medikamente bei Entlassung weiterempfohlen werden.

Datenschutz

Ich weiß, dass ich jederzeit eine bereits erteilte Zustimmung zur Teilnahme an der wissenschaftlichen Studie ohne Angabe von Gründen widerrufen kann, ohne dass mir hierdurch irgendwelche Nachteile entstehen. Daten, die bis zu dem Zeitpunkt des Widerrufs erhoben wurden, werden dann gelöscht.

Meine Angaben werden durch Nummern-Codes verschlüsselt und zum Zweck der Durchführung dieser Studie an die Universitätsmedizin Göttingen, Institut für Allgemeinmedizin, weitergeleitet. Mein Name wird nicht genannt. Eine Entschlüsselung ist nur durch die verantwortlichen Studienmitarbeiter möglich; diese unterliegen selbstverständlich der ärztlichen Schweigepflicht. Dritte erhalten keinen Einblick in die Originalunterlagen. Nach Abschluss der Studie werden meine Daten - entsprechend den Bestimmungen des Datenschutzes - für 10 Jahre gespeichert und anschließend vernichtet. 
Ich habe den Informationstext und eine Kopie dieser Einwilligung erhalten.

Teilnehmer(in): Vor- und Nachname (in Druckbuchstaben)

geb. am

Teilnehmer(in): Unterschrift

Ort, Datum 


\section{Danksagung}

Ich danke meinem Doktorvater Prof. Wolfgang Himmel und dem gesamten Projektteam des Instituts für Allgemeinmedizin der Universitätsmedizin Göttingen. Außerdem möchte ich danken Dipl.-Informatiker Fred Viezens und Luca Hernandez Acosta der Abteilung für Medizinische Informatik der Universitätsmedizin Göttingen für ihre großartige Arbeit für den elektronischen Fragebogen. 


\section{Lebenslauf}

Mein Name ist Freya Sophie Neukirchen. Ich wurde am 21.09.1990 als Kind der Eheleute Inge Gisela Grothus und Kurt Albrecht Neukirchen und als Schwester von Lea Nora Neukirchen in Bremen geboren. In Bremen aufgewachsen, erlangte ich am 19.06.2009 meine allgemeine Hochschulreife am Kippenberg-Gymnasium zu Bremen.

Zum Wintersemester 2009/2010 begann ich ein Studium in Business Administration mit dem Nebenfach Sinologie an der Eberhard-Karls-Universität zu Tübingen. Zum Sommersemester 2011 wechselte ich an derselben Universität zum Studium der Medizin und legte im März 2013 dort die medizinische M1-Prüfung ab.

Ab Sommersemester 2013 setzte ich mein Medizinstudium an der Georg-August-Universität zu Göttingen fort. Im April 2017 legte ich meine M2-Prüfung ab und beendete das Studium der Medizin im Juni 2018 mit einer erfolgreichen M3-Prüfung.

Die vorliegende Arbeit fertigte ich am Institut für Allgemeinmedizin der Georg-AugustUniversität zu Göttingen an unter der Leitung von Herrn Prof. Dr. disc. pol. Wolfgang Himmel. 